\title{
A Simulation Approach to the Performance Evaluation of a Broadband Indoor Wireless Network
}

\author{
by
}

\section{Subodh S. Parulekar, B. E.}

\author{
A thesis submitted to the \\ Faculty of Graduate Studies and Research \\ in partial fulfillment of \\ the requirements for the degree of \\ Master of Engineering \\ Ottawa-Carleton Institute for Electrical Engineering \\ Faculty of Engineering \\ Department of Systems and Computer Engineering \\ Carleton University \\ November, 1996 \\ (C) Copyright \\ 1996, Subodh S. Parulekar
}


National Library

of Canada

Acquisitions and

Bibliographic Services

395 Weilington Street

Ottawa ON KIA ON4

Canada
Bibliothèque nationale

du Canada

Acquisitions et

services bibliographiques

395, rue Wellington

Ottawa ON K1A ONA

Canada
Your tile Votre reterence

Our file Notre relérence
The author has granted a nonexclusive licence allowing the National Library of Canada to reproduce, loan, distribute or sell copies of this thesis in microform, paper or electronic formats.
The author retains ownership of the copyright in this thesis. Neither the thesis nor substantial extracts from it may be printed or otherwise reproduced without the author's permission.
L'auteur a accordé une licence non exclusive permettant à la

Bibliothèque nationale du Canada de reproduire, prêter, distribuer ou vendre des copies de cette thèse sous la forme de microfiche/film, de reproduction sur papier ou sur format électronique.

L'auteur conserve la propriété du droit d'auteur qui protège cette thèse. $\mathrm{Ni}$ la thèse ni des extraits substantiels de celle-ci ne doivent être imprimés ou autrement reproduits sans son autorisation. 


\section{Abstract}

There has been a rapid growth in several segments of wireless personal communications in recent years. One of the potential applications of wireless communications is to support multimedia applications on a broadband ATM platform. In such a system, remote terminals may be capable of producing broadband real-time traffic such as variable bit-rate video or bursty instantaneous file transfers. In this thesis, we describe a broadband indoor wireless system, where the base stations are connected through an ATM network. The ATM switch provides backbone connectivity among microcells as well as with the outside world. We investigate a broadband indoor wireless network (BIWN) employing time division multiple access (TDMA), based on polling, for dynamic bandwidth allocation. This thesis work aims at evaluating the performance of an indoor wireless network supporting high speed bursty traffic using a developed, simplified simulation approach. The results have been obtained through simulations which involve modeling the indoor radio channel behaviour, traffic heterogeneity and implementing an efficient bandwidth management procedure. The performance of the BIWN as seen by a given user terminal has been simulated. 


\section{Acknowledgment}

I am deeply indebted to Prof. Hafez for his knowledgeable supervision. His invaluable guidance and encouragement have contributed significantly towards the success of my thesis. I am also grateful to Prof. Dave Falconer for his helpful suggestions and tips especially during the rudimentary phase of my thesis work.

I have greatly benefited from Naser's and Ashraf's advise on technical issues during the course of my thesis. I am thankful to Prof. Lambadaris, Prof. Devetsikiotis, Sibel and all members of the Broadband Networks Laboratory, Dept. of Systems Engineering, for providing me with video data which was used in my simulations. I am grateful to MIL3 Inc. for giving me an opportunity to use the OPNET simulation package through the University Consortium Agreement. Parsya, Raju and Shekhar need a special mention for their prompt help in every possible matter and for making my stay in the Simulations and Communications lab an interesting one. I am also thankful to Naren for helping me out with the various problems I encountered with the department network.

My special thanks to all my friends at Carleton for making my stay at school a thrilling experience. I consider myself fortunate to have a friend like Praveen, who was there to help me from my first day in Ottawa, and gave me a good start at Carleton! I also appreciate the help rendered by my friends, Ramu and Prasad, on both the academic and non-academic fronts. I am thankful to Ashutosh for his selfless friendship and for being a helpful and a patient roommate. I cannot forget to thank the ambience of my lab at Carleton for infusing enthusiasm in me to conduct my research in an efficient manner.

Above all, I thank my mom, dad and my sister for their love, support and inspiration. 


\section{TABLE OF CONTENTS}

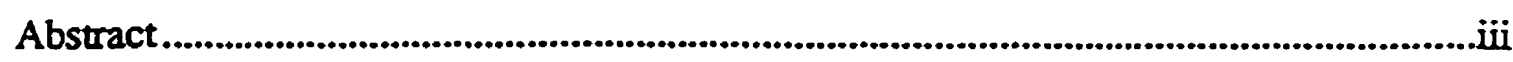

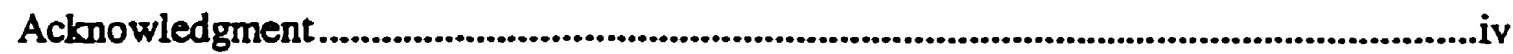

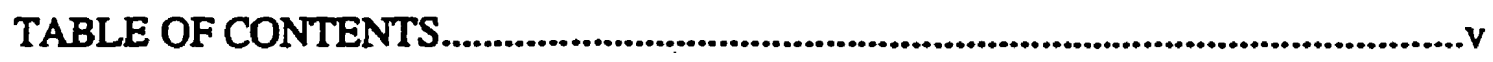

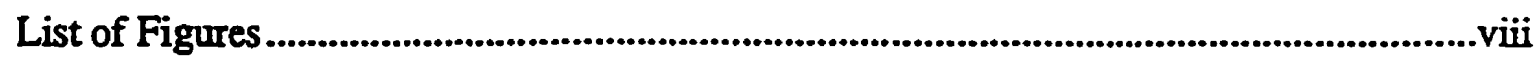

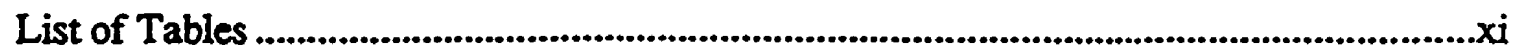

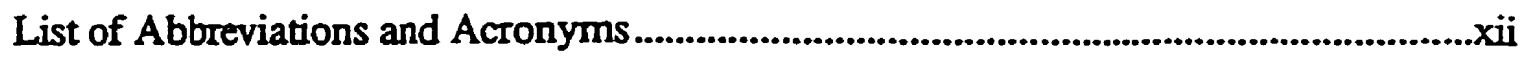

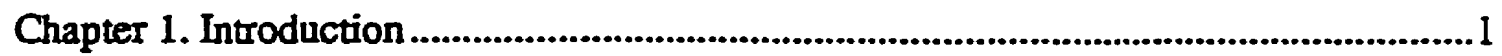

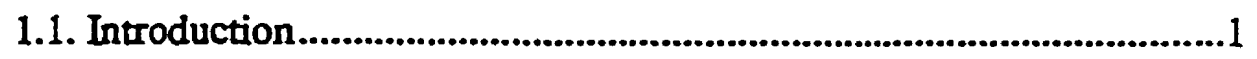

1.2. Thesis Objective....................................................................1

1.3. Organization of the Thesis Report ..................................................2

Chapter 2. Indoor Wireless Communications ......................................................4

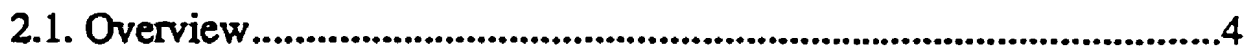

2.2. Requirements ........................................................................5

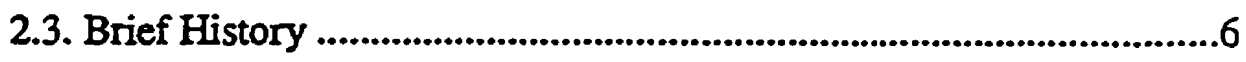

2.4. Millimeter (mm) Wave Link...........................................................11

2.5. Multiple Access Techniques .......................................................12

2.6. Wireless Access to a Broadband ATM LAN......................................13

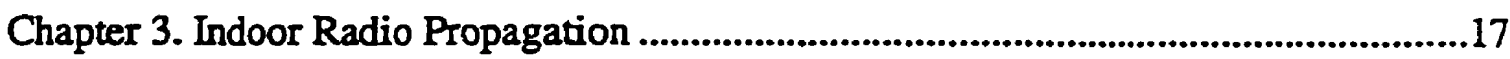

3.1. The Channel Characterization.......................................................17

3.2. Improving SNR at the receiver ...................................................19

3.3. The Channel Model.......................................................................20

Chapter 4. System Description .............................................................................26

4.1. System Description ................................................................27 


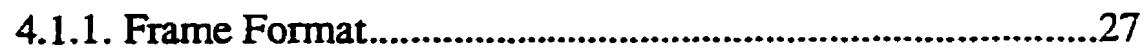

4.2. Multimedia Services and Their Requirements....................................31

4.2.1. Real-Time Services...........................................................32

4.2.2. Modeling of VBR Video Traffic..........................................33

4.2.3. Non-Real-Time Services....................................................34

4.2.4. Multimedia Traffic Modeling .............................................34

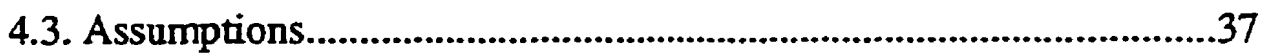

4.3.1. Arrival Process Characteristics..........................................37

4.4. Modeling of Self-Similar Variable Bit Rate Compressed Video.......38

Chapter 5. Simulation Technique and Discussions.........................................................42

5.1. Description of Simulation: 'The Single Terminal Approach' ...........43

5.1.1. The Buffer management : ......................................................45

5.1.2. Multiple Access Time Window: ……….............................45

5.1.3. The Channel Block: .................................................................49

5.1.4. The VBR Video Traffic Source: ...........................................49

5.1.5. Multiple Access Scheme.....................................................51

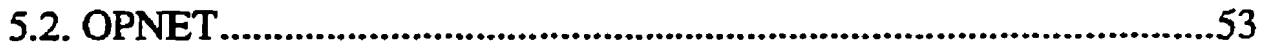

5.2.1. Model Development: .........................................................54

5.2.2. Simulation Execution:...........................................................54

5.2.3. Results Analysis:................................................................54

5.3. Simulations with VBR Video sources based on ON/OFF Markov MiniSources Model .57

5.3.1. Effect of variation of the minimum guaranteed bandwidth. 62 5.3.2. Effect of variation of the Maximum Transfer Delay.........71

5.4. Simulations with VBR MPEG Compressed Video Data exhibiting selfsimilar characteristics..................................................................75 
5.4.1. Effect of variation of the Maximum Transfer Delay. ........76

5.4.2. Effect of variation of Channel Parameters..........................76

5.4.3. Effect of Network Capacity ...........................................82

5.4.4. Effect of the nature of the given video source...................85

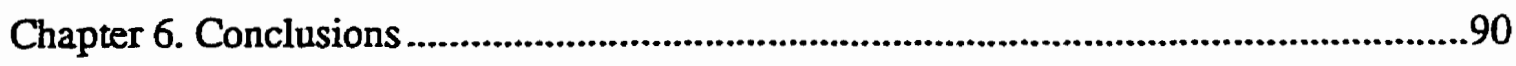

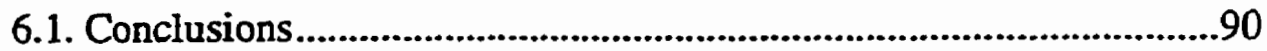

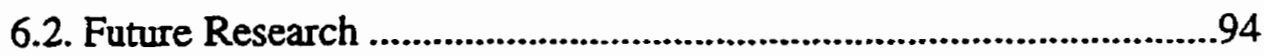

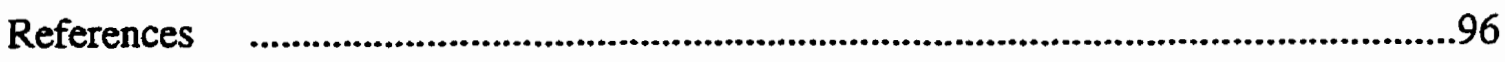




\section{List of Figures}

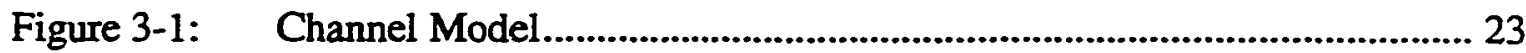

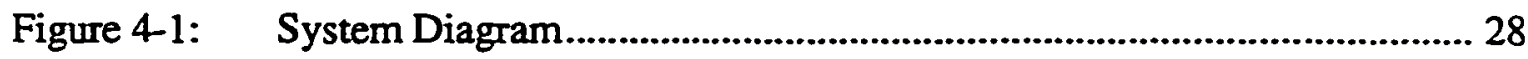

Figure 4-2: The structure of a TDMA frame ...................................................... 30

Figure 4-3: The ON-OFF Markov mini-sources model for VBR traffic.................. 36

Figure 4-4: Autocorrelation of bytes per frame in VBR MPEG video.................... 40

Figure 5-1: The System As Seen By A Given Terminal ....................................... 46

Figure 5-2: The Multiple Access Channel Window ............................................... 48

Figure 5-3: The Channel Model......................................................................... 50

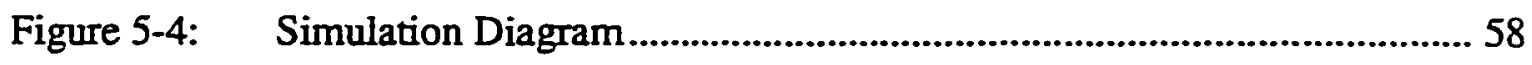

Figure 5-5: Cell Loss Probability versus Utilization for a VBR Video Source modelled with 20 On/Off Mini-Sources (Ns), $A R=0.375$, Mean ON duration $=0.033$ $\mathrm{sec}$ and $\mathrm{MBR}=2.06 \mathrm{Mbps}$. MTD $=33 \mathrm{msec}, \mathrm{CFR}=4.94 \% \ldots \ldots \ldots \ldots . . .64$

Figure 5-6: Mean Buffer Occupancy versus Utilization for a VBR Video Source modelled with 20 On/Off Mini-Sources (Ns), $A R=0.375$, Mean ON duration = $0.033 \mathrm{sec}$ and $\mathrm{MBR}=2.06 \mathrm{Mbps}$. MTD $=33 \mathrm{msec}, \mathrm{CFR}=4.94 \% \ldots . . .65$

Figure 5-7: Mean Waiting Time versus Utilization for a VBR Video Source modelled with $20 \mathrm{On} / \mathrm{Off}$ Mini-Sources (Ns), $\mathrm{AR}=0.375$, Mean ON duration $=0.033$ $\mathrm{sec}$ and $\mathrm{MBR}=2.06 \mathrm{Mbps}$. MTD $=33 \mathrm{msec}, \mathrm{CFR}=4.94 \%$. 66

Figure 5-8: Cell Loss Probability versus Utilization for a VBR Video Source modelled with $20 \mathrm{On} / \mathrm{Off}$ Mini-Sources (Ns), $\mathrm{AR}=0.375$, Mean ON duration $=0.033$ $\mathrm{sec}$ and $\mathrm{MBR}=2.06 \mathrm{Mbps}$. $\mathrm{MTD}=40 \mathrm{msec}, \mathrm{CFR}=4.94 \%$. 68

Figure 5-9: Mean Buffer Occupancy versus Utilization for a VBR Video Source modelled with $20 \mathrm{On} / \mathrm{Off}$ Mini-Sources (Ns), $\mathrm{AR}=0.375$, Mean ON duration = $0.033 \mathrm{sec}$ and $\mathrm{MBR}=2.06 \mathrm{Mbps}$. MTD $=40 \mathrm{msec}, \mathrm{CFR}=4.94 \%$..... 69

Figure 5-10: Cell Loss Probability versus Utilization for a VBR Video Source modelled 
with 20 On/Off Mini-Sources (Ns), $\mathrm{AR}=0.375$, Mean ON duration $=0.033$ sec and $\mathrm{MBR}=2.06 \mathrm{Mbps}$. MTD $=40 \mathrm{msec}, \mathrm{CFR}=5.37 \%$. .70

Figure 5-11: Cell Loss Probability versus Utilization for a VBR Video Source modelled with 20 On/Off Mini-Sources (Ns), $A R=0.375$, Mean ON duration $=0.033$ $\sec$ and $\mathrm{MBR}=2.06 \mathrm{Mbps}$. $\mathrm{Tmin}=20, \mathrm{CFR}=4.94 \%$.

Figure 5-12: Mean Buffer Occupancy versus Utilization for a VBR Video Source modelled with 20 On/Off Mini-Sources (Ns), $A R=0.375$, Mean ON duration = $0.033 \mathrm{sec}$ and $\mathrm{MBR}=2.06 \mathrm{Mbps}$. $\mathrm{Tmin}=20, \mathrm{CFR}=4.94 \%$. .73

Figure 5-13: Mean Waiting Time versus Utilization for a VBR Video Source modelled with $20 \mathrm{On} / \mathrm{Off}$ Mini-Sources (Ns), $\mathrm{AR}=0.375$, Mean $\mathrm{ON}$ duration $=0.033$ $\sec$ and $\mathrm{MBR}=2.06 \mathrm{Mbps}$. $\mathrm{Tmin}=20, \mathrm{CFR}=4.94 \%$. .74

Figure 5-14: Cell Loss Probability versus Utilization for VBR MPEG Compressed Video Source. $M B R=1.145$ Mbps, burstiness ' $b$ ' $=8.83$. $T \min =30, C F R=$ $4.94 \%$. .77

Figure 5-15: Cell Loss Probability versus Utilization for VBR MPEG Compressed Video Source. $\mathrm{MBR}=1.145 \mathrm{Mbps}$, burstiness ' $b$ ' $=8.83$. $T \mathrm{~min}=30, \mathrm{MTD}=$ $33 \mathrm{msec}$. Tmin $=30$, MTD $=33$ msec.

Figure 5-16: Mean Buffer Occupancy versus Utilization for VBR MPEG Compressed Video Source. MBR $=1.145 \mathrm{Mbps}$, burstiness ' $b$ ' $=8.83$. Tmin $=30$, MTD $=33 \mathrm{msec}$. .80

Figure 5-17: Mean Waiting Time versus Utilization for VBR MPEG Compressed Video Source. $\mathrm{MBR}=1.145 \mathrm{Mbps}$, burstiness ' $b$ ' $=8.83$. $T \min =30, \mathrm{MTD}=33$ msec. .81

Figure 5-18: Cell Loss Probability versus Utilization for VBR MPEG Compressed Video Source. $\mathrm{MBR}=1.145 \mathrm{Mbps}$, burstiness ' $b$ ' $=8.83$. $\mathrm{Tmin}=30, \mathrm{CFR}=$ $1.03 \%, \mathrm{MTD}=33 \mathrm{msec}$. .83

Figure 5-19: Mean Buffer Occupancy versus Utilization for VBR MPEG Compressed Video Source. MBR $=1.145 \mathrm{Mbps}$, burstiness ' $b$ ' $=8.83$. $T$ min $=30$, CFR $=1.03 \%, \mathrm{MTD}=33 \mathrm{msec}$. .84

Figure 5-20: Mean Waiting Time versus Utilization for VBR MPEG Compressed Video Source. $\mathrm{MBR}=1.145 \mathrm{Mbps}$, burstiness ' $b$ ' $=8.83$. Tmin $=30, \mathrm{CFR}=1.03$ $\%, \mathrm{MTD}=33 \mathrm{msec}$. .86

Figure 5-21: Cell Loss Probability versus Utilization for VBR MPEG Compressed Video Source. $C F R=4.94 \%, M T D=33 \mathrm{msec}$.

Figure 5-22: Mean Buffer Occupancy versus Utilization for VBR MPEG Compressed 


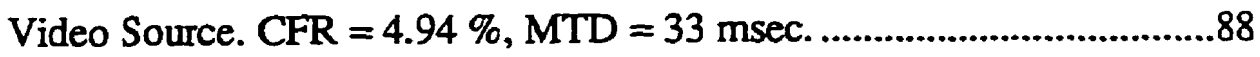

Figure 5-23: Mean Waiting Time versus Utilization for VBR MPEG Compressed Video Source. CFR $=4.94 \%$, MTD $=33$ msec................................................89 


\section{List of Tables}

Table 2-1: Requirements of typical multimedia services ......................................... 14

Table 5-1: Video Sources Based On On/Off Markov Mini-Sources Model............ 60

Table 5-2: Channel Parameter Values (Channel Model A)...................................... 61

Table 5-3: Channel Parameter Values (Channel Model A1)..................................... 62

Table 5-4: Video Sources Based on VBR Compressed Video Data ....................... 75

Table 5-5: Channel Parameter Values (Channel Model B)........................................ 78 


\section{List of Abbreviations and Acronyms}

AR

ATM

BIWN

CBR

CDMA

CFR

CLP

FDMA

LAN

MBO

Mbps

MBR

MTD

MWT

NRTT

PR

RF

RTT

SNR

TDMA

$T_{\min }$

VBR
Activity Ratio

Asynchronous Transfer Mode

Broadband Indoor Wireless Network

Constant Bit Rate

Code Division Multiple Access

Channel Failure Rate

Cell Loss Probability

Frequency Division Multiple Access

Local Area Network

Mean Buffer Occupancy

Mega-bits per second

Mean Bit Rate

Maximum Transfer Delay

Mean Waiting Time

Non Real-Time Traffic

Peak Rate

Radio Frequency

Real Time Traffic

Signal to Noise Ratio

Time Division Multiple Access

Minimum Guaranteed Bandwidth

Variable Bit Rate 


\section{Chapter 1 \\ Introduction}

\subsection{Introduction}

The past few years have witnessed a rapid growth in the demand for communications services. Communications networks have become the most important global stimulator of economic growth. The goal of wireless communication is to allow the user access to the utilities of the global network regardless of location or mobility. The innate need to communicate while on the move necessitates a tetherless access to a communication network. This has caused a rapid increase in the sales of cordless telephones and increasing support for mobile cellular systems. Successful implementation of mobility management in cellular communications, availability of low-power lightweight mobile terminals, and emerging intelligent network technologies has resulted in significant activities in wireless personal communications.

\subsection{Thesis Objective}

There is growing interest in wireless in-building communications systems. The Indoor wireless networks bring about attractive advantages such as easy deployment, reduced wiring in new buildings, flexibility of changing or creating various communications services in existing building without the need for expensive, time consuming rewiring [1]. 
Also, the users' phones are freed from cords tethering them to specific locations within the buildings, thus allowing for portability and mobility. Most existing networks are focussed on voice and data applications. The rapid development of personal communications systems is widening the scope of supported applications. Efforts are underway to incorporate multimedia capability into the mainstream of personal communications services. As a result, remote terminals may be capable of producing broadband real-time traffic such as variable bit-rate video or bursty data transfers. A broadband indoor wireless system will provide untethered connections between numerous scattered multimedia terminals.

Due to the availability of wide uncongested bandwidth in the millimeter wave band (above $20 \mathrm{GHz}$ ), it could be a good transmission medium to meet the requirement of broadband services. As part of a series of efforts targeting future Broadband Indoor Wireless Networks (BIWNs), this work investigates a broadband indoor wireless system which employs Time Division Multiple Access (TDMA) based on polling for dynamic bandwidth allocation. We describe a broadband indoor wireless system where the base stations are connected through a fiber-based ATM network. The ATM switch provides backbone connectivity among microcells as well as with the outside world. The work aims at evaluating the performance of an indoor wireless network supporting high speed bursty traffic using a developed, simplified simulation approach. The work encompasses two tasks that are required to be executed in order to meet the desired goal, viz. developing a simulation tool to evaluate the performance of a BIWN, and secondly to develop a video traffic model for use in the simulation tool.

\subsection{Organization of the Thesis Report}

This thesis is structured as a series of concatenated modules. Chapter 2 provides an overview of indoor wireless communications, the future requirements this system calls for, 
and brief information about the evolution of a broadband indoor wireless networking system. This chapter also includes sections that touch upon various issues of relevance to a millimeter-wave link and multiple-access techniques in general. The chapter concludes with a discussion about the new trend towards wireless access to a broadband ATM LAN.

Chapter 3 discusses in detail the characterization of indoor radio propagation. It covers the important techniques used to improve SNR at the receiver. It finally discusses the indoor channel model that has been incorporated in our simulation tool. This section highlights the role played by the constituents of the channel model, viz. fading, shadowing and interference, in an indoor wireless system.

Chapter 4 starts with a discussion of the principal issues of a broadband indoor wireless system. It contains a description of the system used for our simulations, which includes the system layout and details of the multiple access scheme employed. The chapter further explains the characteristics of multimedia services, their classification and their modeling techniques.

Chapter 5 describes the simulation technique employed for the performance evaluation of the BIWN under consideration. The key results have been presented and analyzed in this chapter.

Conclusions are presented in Chapter 6. 


\section{Chapter 2 \\ Indoor Wireless Communications}

Indoor radio communication systems are becoming increasingly important for extending communication services within the work place. Indoor wireless systems can be classified into three kinds: (1) cordless telephone systems; (2) in-building cellular systems; and (3) local area networks (LANs) [2]. This chapter presents some of the significant features of indoor wireless communications.

\subsection{Overview}

The indoor environment covers a wide range of structures characterized by proximity and communality of purpose [3]. Thus the term 'indoor environment' encompasses:

(i) office or apartment towers or smaller structures

(ii) campuses such as hospitals, universities, and laboratories

(iii) building complexes

(iv) factories

(v) residential houses

(vi) shopping centers

Indoor communications, supports the following applications:

(i) Communication systems: multi-function telephones, electronic or voice mail, 
FAX, broadcast TV etc.

(ii) Office automation systems. CAD/CAM, distributed databases, graphics and video, text composition, video conferencing, computer communication facilities etc.

(iii) Building automation systems: security/emergency services, automated airconditioning and lighting, card controlled access, hallway information system etc.

In an indoor communications system, the main services to be integrated include but are not limited to: (i) telephone services, (ii) computer communication services, (iii) security/ emergency services, and (iv) video services.

In summary, indoor communications are characterized by variable user density, variable bit rate and variable traffic requirements.

\subsection{Requirements}

An indoor wireless system calls for the following future requirements:

- Improved signal quality and reliability

- Incorporation of data, voice and video services

- Small and lightweight, low power consumption terminals

- Automatic channel assignment, control and monitoring in an integrated environment of wired and wireless systems

- Efficient coordinated use of radio spectrum 


\subsection{Brief History}

The number of users for indoor use is rapidly growing in offices, manufacturing floors, shopping areas and warehouses. In the near future, one expects to see several terminals clustered within a small indoor area. To avoid cable pulling to many locations, and to provide flexibility in the placement of terminals, wireless communications for indoor applications is appealing. The range of coverage for indoor radio communications is small, which leaves many options open for the transmission technology. Existing products can be divided into five categories based on the transmission technology and the access technique used by them [4]: diffused IR (DFIR), directed beam IR (DBIR), standard radio (RF), direct sequence spread spectrum (DSSS), and frequency hopping spread spectrum (FHSS). These technologies and techniques have evolved around the availability of the channel and the suitability of the transmission technique to provide a high data rate link in the wireless media.

In the late 1970s IBM Laboratories in Ruschlikon, Switzerland published the results of their experimental work on the design of a wireless indoor network using diffused $\mathbb{R}$ technology that was envisioned to be used on manufacturing floors [5]. Around the same time, another experiment was performed at Hewlett-Packard laboratories [6] examining the use of direct sequence spread spectrum technology for wireless inter-terminal communications. The data rates experimented by both methods were around $100 \mathrm{~Kb} / \mathrm{s}$ [7]; however neither of the projects were tumed into a commercial product due to certain impediments. However, these works triggered further research in high speed wireless indoor communications. Hewlett-Packard laboratories and others developed directedbeam IR networks [8], [9]; Motorola's Codex worked on a wireless LAN using ordinary radio modems at $1.7 \mathrm{GHz}$ and petitioned the Federal Communications Commission (FCC) for that band [10], none of which tumed into a commercial product. 
Development of commercial wireless LAN products entered a more serious phase after the announcement of the Industrial, Scientific and Medical (ISM) bands by the FCC and Industry Canada in May 1985 [4]. By 1990, wireless LAN products using DSSS (direct sequence spread spectrum) in the ISM bands, licensed radio at 18-19 GHz [11], and IR technology appeared in the market. These products represented the first commercial implementation of wireless LANs.

In an indoor application, infrared does not interfere with existing radio frequency system and there is no FCC regulation for this particular band of electromagnetic spectrum. The FCC is only concemed with radio frequencies below $300 \mathrm{GHz}$. Infrared radiation is essentially restricted to a room in which it is generated; it cannot be detected outside a room and will not interfere with similar systems in neighbouring offices. However, infrared is not suitable for very large offices or rooms with double blind corners. The data rate and number of users in infrared systems is also restricted as compared to radio frequency systems. The infrared indoor radio channels are very sensitive to the room size, physical shape of the room, location of the objects in the room, sunlight and fluorescent lights, and so on. These factors stand as serious obstacles to infrared systems for applications other than slow-speed systems in open working environments [12].

For indoor communications using radio frequency, the UHF band from $300 \mathrm{MHz}-3 \mathrm{GHz}$, is suitable. Several radio propagation studies at $900 \mathrm{MHz}$ within an office setting were made in 1982. The experimental results show a maximum received power fluctuation of around 25-30 dB. An efficient technique used to combat signal fading is to increase the transmission bandwidth using spread spectrum techniques. Spread spectrum can reduce the interference of the broadcast spread spectrum RF signal with any existing system and render the signals undetectable outside the office building.

UHF spread spectrum indoor wireless communications systems suffer from critical 
bandwidth and spectrum reuse shortcomings that seem likely to prevent them from expanding beyond niche applications. Another serious problem for UHF is that RF energy at these frequencies tends to propagate through and around obstacles, reaching beyond the confines of the network it serves. It may be good for other applications, but it can't be contained within the confines of a microcell and therefore limits the spectrum reuse, a phenomenon of immense importance for the viability of an indoor wireless system.

Cellular radio can be regarded as the earliest form of wireless "personal communications" [13]. Cellular system design was pioneered during the '70s by Bell Laboratories in the United States, and the initial realization is known as AMPS (Advanced Mobile Phone Service). Systems similar to AMPS were soon deployed internationally. FDMA (frequency division multiple access) was used for spectrum sharing. Starting in the 1980's, first generation cordless telephones were developed for residential voice services. These systems used analog frequency modulation for speech transmission. The most serious drawback of those systems is the operating range which is limited to tens of meters from a single base station. Another big problem is vulnerability to interference from other cordless phones. Most first generation cordless phones have access to only one channel and the user can do nothing to avoid interference from someone nearby using the same channel. Almost all of the cordless telephones that have access to several channels rely on manual selection, by the user, to avoid interference.

The development of low-rate digital speech coding techniques and the continuous increase in the device density of integrated circuits, have made completely digital secondgeneration systems viable. Digitization allows the use of TDMA (time division multiple access) and CDMA (code division multiple access) as alternatives to FDMA [14]. To overcome the limitations of first generation technology and satisfy the exploding demand for wireless access to communication networks, second generation cordless phones came 
into service in recent years. Some of the developed cordless telephone standards include CT2, CT2t, and DECT. These systems employ digital speech transmission and have dedicated channels for the control of information between the base station and a portable during a call. In such a system, a cordless phone is able to communicate with many base stations and automatically selects the best available radio channel. They are designed to be interconnected to ISDN facilities. CT2 operates in the frequency band $864-868 \mathrm{MHz}$, CT2+ at $950 \mathrm{MHz}$, while DECT occupies a frequency band at $1.88-1.90 \mathrm{MHz}$ [15].

At the beginning of 1990's, the research and development performed by Motorola's Wireless Inbuilding Network technology group addressed the user requirements and the technical challenges of the indoor environment. One of the major conclusions is that $18 \mathrm{GHz}$ radio is an appropriate choice for indoor communications [12]. The properties of $18 \mathrm{GHz}$ band make it a good choice for networked cellular communications within buildings. This frequency band offers sufficient bandwidth to handle the higher data speeds expected of future systems, as well as propagation characteristics suited to low power in-building cellular networks. The ALTAIR system supporting bit rates of $15 \mathrm{Mbps}$ was developed by Motorola in 1991 [15]. A combination of 60 degrees antenna sectorization in the horizontal plane and selection diversity is used to combat multipath effects. The selection of antenna beams in the transmitter and receiver is made by a central processor which monitors the signal quality in all 36 possible combinations (six beams at the transmitter and six at the receiver). The width of each beam in the vertical plane is also 60 degrees and therefore it does not provide complete coverage in azimuth. Since the ALTAIR system is not designed to be portable, the base station units and the user units are mounted at fixed angles which take into account the limited antenna coverage.

Starting in 1988, there was some research interest in mm-wave channels. In particular the mm-wave band around $60 \mathrm{GHz}$ was the subject of much investigation. Extensive research 
activities are being conducted in Canadian universities under the funding of CITR. The objective is to investigate various aspects of the mm-wave band, and ultimately develop an ATM based wireless LAN. Studies have been carried out to estimate bit rate and performance requirements of broadband wireless links to LANs that may accommodate a wide range of multimedia traffic. These indicate that users may require peak bit rates on the order of 20 to $30 \mathrm{Mbps}$ for high resolution compressed video, although the instantaneous bit rate required is variable [16]. The choice of multiple access strategy and microcellular architecture is heavily influenced by technology considerations. A simulation study was completed to investigate indoor wireless communications in the 20$60 \mathrm{GHz}$ band using TDMA multiple access [17]. There are some reported results on the studies of mm-wave system protocols for bit rates of $100 \mathrm{Mbps}$ or more in European universities [18], [19], [20], [21]. Some important functional differences between these present wireless systems/proposals and future indoor wireless communications are:

- Attempt to use $20 \mathrm{GHz}$ to $60 \mathrm{GHz}$ band (mm-wave bands)

- Integrated voice, data and video services

- Higher data rate, data service rate up to $155.2 \mathrm{Mbps}$ (OC-3 ATM-SONET rate) will be attempted

Standards for wireless LANs are being developed under IEEE 802.11 in the United States and ETSI/RES10 in Europe (known as HIPERLAN, for High Performance Radio LAN). Both standards are intended for rates exceeding $1 \mathrm{Mbps}$ and have asynchronous packet transmission as the dominant mode.

\subsection{Millimeter (mm) Wave Link}

The millimeter-wave band refers to the frequency band whose wavelength is in the order 
of millimeters e.g. 20-60 GHz. Such a link looks to be a favorable choice for indoor communication. The millimeter waves possess the following important characteristics [22]: a) travel mostly by line of sight, b) highly attenuated by atmosphere or rain, c) various atomic, molecular, and nuclear resonance occur at microwave frequencies and are utilized in remote sensing, medical diagnosis and treatment, and cooking methods.

The $20 \mathrm{GHz}$ to $60 \mathrm{GHz}$ band (mm-wave) looks to be a favorable choice for indoor communication requirements because:

(i) there is sufficient bandwidth for the high data rates required in today's wired local area networks

(ii) current usage is sparse

(iii) device dimensions for these frequencies are small

(iv) the short range propagation of EM waves at these frequencies is suitable for micro-cellular or pico-cellular system architectures

(v) the favorable regulatory environment. High attenuation by exterior walls along with short range propagation at lower transmitter power results in confinement of the signals.

Compared to conventional UHF bands, the advantage of mm-wave channel is the availability of a high information transport capacity. In addition, this frequency reuse is possible over very short distances, for example in adjacent rooms of an office floor. Because of these features and recent advances in GaAs technology, the mm-wave frequency band should be identified as a possible bearer for broadband data and video services as offered by fibre optic networks. The broadband nature is especially relevant because of the advent of BISDN (Broadband Integrated Services Digital Network). 


\subsection{Multiple Access Techniques}

Multiple access techniques are used to provide multiple simultaneous users with radio links of good transmission quality. The three basic multiple access techniques in fixed assignment are time division multiple access (TDMA), frequency division multiple access (FDMA) and code division multiple access (CDMA).

In FDMA, each user is assigned a frequency channel for duplex communications. FDMA, although presently used in narrowband wireless systems [23], is recognized to be unsuitable for an indoor broadband wireless network (IBWN). To accommodate services with a wide range of arrival characteristics and bitrates and to perform dynamic bandwidth allocation, FDMA requires fast changes in channel bandwidth which is very difficult to implement. Besides, the relatively high cost of generating carriers at millimeter waves, tends to discourage the use of FDMA. CDMA is not favoured because of its bandwidth and complex power control requirement. [24]. Further, supporting the chip rate for high bit rate services may be difficult to implement; so CDMA has the disadvantage of relatively low peak transmission bit rate compared to TDMA, which is a grave problem in multimedia communications. A non-power controlled version of CDMA/ALOHA, with low processing gain was found to be less efficient than a TDMA-based protocol for broadband $\mathrm{mm}$-wave access [25]. Power control did not improve the efficiency sufficiently to justify its higher cost and complexity [26]. A TDMA-based multiple access method is considered appropriate for the indoor wireless system under consideration.

\subsection{Wireless Access to a Broadband ATM LAN}

The variety of applications in telecommunications have been envisaged to increase significantly. General purpose, modular, and layered concepts based on standardized network and service building blocks and suitable platforms will provide the universal and 
economic solutions required. These concepts will be supported by the uniform and bit rate-independent asynchronous transfer mode (ATM). Broadband and multimedia communications applications are typical examples of the growing qualitative and quantitative requirements placed on telecom networks and services. Comprising data, text, graphics, image, video, and audio communication, multimedia applications are gaining in importance with increasing demand and with the advent of powerful personal computers and workstations, high-performance central computer systems, high capacity storage devices, and high speed networks. Henceforth, applications with mixes of different information types, service components, and services will play a growing role. Different traffic-flow characteristics like continuous traffic with constant or variable bit rates or bursty traffic have to be handled in the network. Some of the expected services to be offered by a multimedia terminal in broadband networks, are listed in Table 2-1, taken from references [27], [28], [29], [30], [31]. It is apparent that the requirements of the listed services are very diverse. Real-time applications, incorporating voice and video (isochronous services), are mostly sensitive to excessive transmission delay, while nonreal-time data applications (asynchronous services) have a very low cell loss tolerance. ATM, as a globally standardized principle for multiplexing and switching, will contribute to the universality and flexibility in telecommunications significantly. The unified packaging of all information types into cells of fixed length offers great benefits [32]: connections, bit rates and qualities on demand, simple service integration, and capacity control in the network according to current needs. 
Table 2-1: Requirements of typical multimedia services

\begin{tabular}{|c|c|c|c|c|c|c|}
\hline & Service type & $\begin{array}{l}\text { Bursti } \\
\text {-ness }\end{array}$ & $\begin{array}{l}\text { Burst } \\
\text { duration }\end{array}$ & $\begin{array}{l}\text { Required } \\
\text { bandwidth }\end{array}$ & $\begin{array}{l}\text { Maximum } \\
\text { cell error }\end{array}$ & Maximum \\
\hline & $\begin{array}{l}\text { VBR (CBR) } \\
\text { Voice }\end{array}$ & $\begin{array}{l}5-15 \\
(1)\end{array}$ & $1 \mathrm{sec}$ & $16-256 \mathrm{kbps}$ & $10^{-4}-10^{-6}$ & $150-250 \mathrm{~ms}$ \\
\hline & $\begin{array}{c}\text { VBR(CBR) } \\
\text { Video Tele- } \\
\text { phony }\end{array}$ & $2-5(1)$ & $30-100 \mathrm{~ms}$ & $64 \mathrm{kbps}$ & $10^{-4}-10^{-6}$ & $150-350 \mathrm{~ms}$ \\
\hline & $\begin{array}{l}\text { VBR (CBR) } \\
\text { Video Con- } \\
\text { ferencing }\end{array}$ & $2-5(1)$ & $30-100 \mathrm{~ms}$ & 64-320 kbps & $10^{-4}-10^{-6}$ & $\begin{array}{l}150-350 \mathrm{~ms} \\
\text { (jitter } 5 \mathrm{~ms} \text { ) }\end{array}$ \\
\hline$\frac{\stackrel{\mathscr{\Xi}}{\Xi}}{\underline{\underline{E}}}$ & $\begin{array}{c}\text { VBR(CBR) } \\
\text { MPEG } \\
\text { NTSC Video }\end{array}$ & $2-5(1)$ & $33 \mathrm{~ms}$ & 1-4 Mbps & $10^{-4}-10^{-6}$ & $40 \mathrm{~ms}$ \\
\hline & $\begin{array}{c}\text { VBR (CBR) } \\
\text { HDTV } \\
\text { NTSC Video }\end{array}$ & $2-5(1)$ & $33 \mathrm{~ms}$ & $20-150 \mathrm{Mbps}$ & $10^{-4}-10^{-6}$ & $40 \mathrm{~ms}$ \\
\hline & $\begin{array}{c}\text { File Transfer } \\
\text { Electronic } \\
\text { Mail }\end{array}$ & 1 & $1 \mathrm{sec}$ & $\begin{array}{l}9.6 \mathrm{kbps}- \\
2 \mathrm{Mbps}\end{array}$ & $10^{-9}$ & $10-100 \mathrm{sec}$ \\
\hline & $\begin{array}{l}\text { Medical X- } \\
\text { ray } \\
\text { Still Image }\end{array}$ & $25(1)$ & $1 \mathrm{sec}$ & 1-10 Mbps & $10^{-9}-10^{-12}$ & $1-5 \mathrm{sec}$ \\
\hline 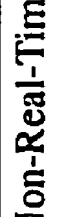 & $\begin{array}{l}\text { Document } \\
\text { Retrieval } \\
\text { Data on } \\
\text { Demand }\end{array}$ & $\begin{array}{l}40- \\
200\end{array}$ & $40-250 \mathrm{~ms}$ & $\begin{array}{c}64 \mathrm{kbps} \\
-5 \mathrm{Mbps}\end{array}$ & $10^{-9}-10^{-12}$ & $1-3 \mathrm{sec}$ \\
\hline
\end{tabular}

As the demand for broadband communications services grows rapidly, the wireless access 
to broadband networks appears as a new research area which attracts attention. A recent forecast for the U.S. market estimates the growth of integrated wireless office systems from zero in 1992 to over $\$ 1-3$ billion in 1997 [33]. Broadband wireless access will afford potentially enormous bandwidth on demand to people on the move, using small portable multimedia terminals. To unite broadband communications with wireless personal communications, and make possible untethered access to a wide range of broadband communications services, such as multimedia document communications, high resolution image or large file transfer, high resolution image or large file transfer, high resolution video teleconferencing, etc., the research and development on a broadband indoor wireless digital communications system capable of supporting ATM for broadband LANs has intensified a frontier and it will affect how we live and interact in the foreseeable future. In [27], an analysis of various bandwidth allocation procedures is carried out.

The cost of wireless access to an ATM LAN, including deployment and equipment costs, must not be much higher than that of tethered access via fibre or copper to individual terminals. Thus, exploiting millimeter wave device technologies to design and realize the various transceiver components in low cost, low power monolithic and/or hybrid form is an important objective. Several factors influence the design of wireless broadband networks such as traffic demand, number of portable users, channel bandwidth, portable traffic fluctuations, cost, complexity etc. and a set of performance measures such as cochannel interference, blocking and transmission quality. It is expected that wireless access to broadband networks will have its initial strongest appeal in office type environments, but that as costs diminish, it will also be heavily used in factories, hospitals, schools and residential environments. 


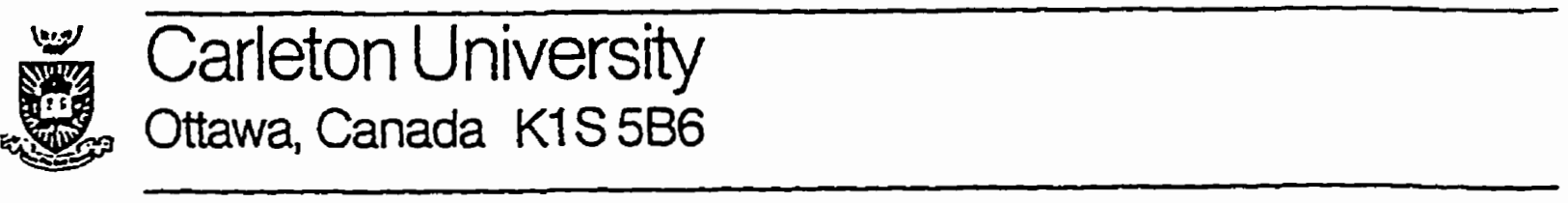

Text complete; leaf $/ 6$ omitted in numbering. 


\section{Chapter 3 \\ Indoor Radio Propagation}

The nature of the indoor channel influences the design of the indoor wireless communication system. The indoor radio channel is subjected to the following impairments:

a) Flat or frequency selective fading arising due to multipath propagation, whereby replicas of the transmitted signal reach the receiver suffering different propagation delays.

b) The local average signal changes, termed shadowing, which is caused by obstruction of the radio path by an impenetrable body.

c) Interference from nearby cells using the same frequency band (termed co-channel interference) and from adjacent frequency bands (termed adjacent channel interference).

\subsection{The Channel Characterization}

For indoor propagation, the radio channel is essentially a linear time varying filter for each point in three dimensional space and can be characterized by multipath components of random time varying amplitude, arrival time and phase sequences as shown next:

$$
h(t, \tau)=\sum_{k=0}^{N(\tau)-1} a_{k}(t) \delta\left(\tau-\tau_{k}(t)\right) e^{j \theta_{k}(t)}
$$


where $t$ and $\tau$ are the observation time and application time of the impulses. Equation 3.1 represents the low pass equivalent representation of the channel. $N(\tau)$ is the number of multipath components, $\left\{a_{k}(t)\right\},\left\{\tau_{k}(t)\right\},\left\{\theta_{k}(t)\right\}$ are the random time varying amplitude, arrival time, and phase sequences, respectively, and $\delta$ is the delta function.

For a slowly varying channel, we have the following stationary (time-invariant) model:

$$
h(\tau)=\sum_{k=0}^{N(\tau)} a_{k} \delta\left(\tau-\tau_{k}\right) e^{j \theta_{k}}
$$

The small scale rapid amplitude fluctuations in absence of a strong received component is commonly modeled by the Rayleigh distribution.

The probability density function of the signal amplitude under Rayleigh fading is given by:

$$
f_{R}(r)= \begin{cases}\frac{r}{\sigma^{2}} e^{\frac{r^{2}}{2 \sigma^{2}}} & r \geq 0 \\ 0 & r<0\end{cases}
$$

where $\sigma^{2}=\frac{1}{2} E\left\{r^{2}\right\}$ is the local mean power. When a strong path exists in addition to the low level scattered paths, the received signal follows the Rician distribution given by the pdf:

$$
f_{R}(r)=\frac{r}{\sigma^{2}} \exp \left\{\frac{r^{2}+v^{2}}{2 \sigma^{2}}\right\} I_{0}\left(\frac{r v}{\sigma^{2}}\right)
$$

where $I_{0}$ is the zeroth-order modified Bessel function of the first kind, $v$ is the magnitude of the strong component and $\sigma^{2}$ is proportional to the power of the "scatter" Rayleigh component. The other well known distributions are: the Nakagami distribution, the Weibull distribution, the Lognormal distribution (used to explain large scale variations of 
signal amplitudes) and the Suzuki distribution (mixture of Rayleigh and Lognormal distributions).

An important parameter in broadband wireless systems is the delay spread (DS), which is the measure of the duration of the channel impulse response. The r.m.s. (root mean square) delay spread is defined as the square root of the second central moment of the power delay profile. It is a good measure of the multipath spread and indicates the potential for intersymbol interference. The median value of the r.m.s. DS observed with a centrally located transmitter has been found to vary from $25 \mathrm{~ns}$ in a medium size office building to about $125 \mathrm{~ns}$ in a large office building [1], [34]. Due to the motion of people and equipment around the low-level portable antennas, the indoor radio channel is nonstationary in time and space. However, due to the absence of rapid motions and high velocities (typical of outdoor channels), the indoor channel's doppler shift is negligible. The indoor radio channel is characterized by severe path losses and sharp changes in the mean signal level as opposed to the outdoor channel.

\subsection{Improving SNR at the receiver}

The following techniques are adopted in order to combat the fading phenomenon:

a) Diversity: This is a widely used technique to mitigate the fading effects. It achieves major improvements in the quality of reception by exploiting the fact that two or more independent samples received at antennas sufficiently spaced apart are uncorrelated. If $p$ is the outage probability (the probability of threshold SNR dropping below the accepted threshold) corresponding to a signal sample, then the probability of getting an unacceptable SNR out of $m$ independent samples is $p^{m}$. Thus with an $m$-branch diversity the fading effect is exponentially reduced by a factor $\mathrm{m}$. The base station either selects the antenna that gives the best performance or suitably processes and combines the signals 
from both antennas to enhance the quality of reception. The diversity reception technique may be applied at the base station, remote terminals or both.

The use of diversity reception enables higher local interference power levels for a given system performance, resulting in higher spectrum efficiency. Diversity may be applied either in frequency, polarization, time or space. The last two are the commonly used techniques.

b) Other techniques: Channel equalization and error correcting coding are two other common techniques used for error rate reduction [24].

\subsection{The Channel Model}

Summing up, the transmitted radio signal in an indoor wireless system, is subjected to:

a) Large scale path loss effects: the average received signal power decreases logarithmically with distance.

b) Log-normal Shadowing: the measured signal level in $\mathrm{dB}$ at a specific TransmitterReceiver separation has a Gaussian distribution about the distance dependent mean signal level.

c) Small Scale Multipath Propagation: Multipath in the radio channel creates small scale fading effects. The three most important effects are:

1. Rapid Changes in signal strength over a small travel distance or time interval.

2. Random frequency modulation due to varying doppler shifts on different multipath signals.

3. Time dispersion (echoes) caused by multipath propagation delays.

d) Co-channel and adjacent channel interference. Interference characteristics are 
determined by fading and dynamic frequency allocation.

A received signal envelope experiences occasional deep fades which results in a burst of errors [35]. During a deep fade, there is a sudden dip in the signal level. The instantaneous signal level shoots down below an acceptable threshold in a very short interval of time. Thus the channel can be modeled as being 'ON' or 'OFF', without considering the representation of the intermediate states of the channel. In [34], the indoor channel has been modeled by two exponentially distributed states, viz. ON and OFF. This model has considered only fading effects. Depending on whether the channel is under a fade or not, it is either in a good state (ON), or a bad state (OFF).

Shadowing effects can be severe in an indoor mm-wave system and must be considered in the channel model. There is a greater probability of the channel recovering late to a 'good' state under shadowing. Shadowing is much slower than the rapid fading process, wherein the channel can be blocked off for a very long period of time.

The indoor channel is affected not just by fading but also by shadowing and interference. All the three phenomena, viz. fading, shadowing and interference must be considered in the channel model. We approximate the indoor channel behaviour by a multi-state ONOFF model in which the OFF state of the channel is further split into sub-states corresponding to shadowing (S1, S2, S3,...), fading ( F1, F2, F3,...) and interference (I1, $12,13, \ldots)$ as shown in Figure 3-1.

- In our system, transmission takes place in cycles, i.e., multiple access frames. The system is clocked and each state (G, S, F, I) corresponds to a multiple access frame duration.

- Presumably, if fading and shadowing appear and disappear with time, it is entirely due to: (i) movement - of transmitter or receiver, or of people or objects in 
their vicinity; and (ii) system measures such as diversity switching.

- The channel condition is assumed to remain unchanged within a cycle.

- Transition from one state of the model to another takes place on a per frame/cycle basis.

- Shadowing (S), Fading (F), and Interference (I) represent the OFF state of the channel. Otherwise, the channel is in the good (ON) state.

- Channel recovers from a bad condition ('OFF' state) either on its own, or due to system intervention. The base station has continuous information about the status of the channel.

- Considering the slow nature of the channel and a short transmission, amplitude variations in the channel response have been neglected.

As shown in the channel model, from a given state, a transition can be made to a further 'OFF' state, or back to the 'good' ('ON') state. A transition to the same state (except in the case of good state) is not possible. The probability of moving further to an 'OFF' state decreases monotonically. In case the channel is in the first state of shadowing (S1), the probability that it would return to a 'good' state is lower than that of returning to the 'good' state from the second state of 'shadowing' (S2) and so on. Assuming the existence of Power Control/Automatic Gain Control, Pathloss has not been considered in the channel model. Further, doppler shift has also been ignored due to the absence of high portable speeds in an indoor environment.

The state labeled " $\mathrm{G}$ " corresponds to the channel being in the "ON" state, while the states labeled Shadow1, Shadow2, Shadow3,..., Fade1, Fade 2.... and I1, I2,.... correspond to the channel being in the "OFF" state. 


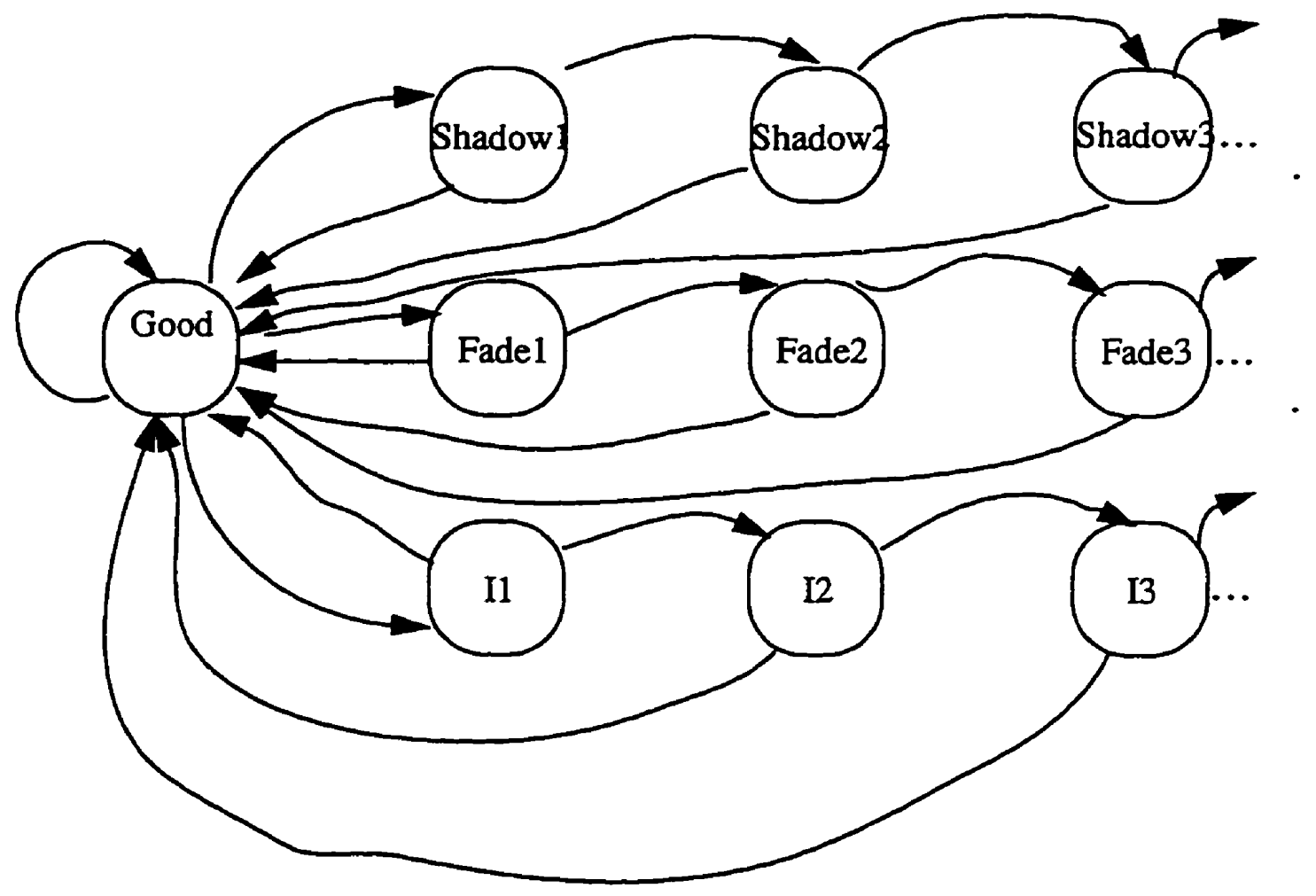

Figure 3-1: Channel Model 
Let " $T_{n}$ " (e.g.: $T=S, F, I ; n=1,2,3, \ldots$ ) be one of the states represented in the model, corresponding to the OFF state of the channel. Assuming that the channel is in state " $G$ ", the channel has a probability $P_{G}$ of being in the state " $G$ ", a probability $P_{G S 1}$ of making a transition to "SI", a probability $\mathrm{P}_{\mathrm{GF} 1}$ of making a transition to "F1" and a probability $\mathrm{P}_{\mathrm{GI}}$ of making a transition to " $\mathrm{Il}$ ". If the channel is assumed to be in one of the " $\mathrm{T}_{\mathrm{n}}$ " states then the channel can either make a transition to " $\mathrm{T}_{\mathrm{n}+1}$ " with a probability $\mathrm{P}_{\mathrm{Tn}+1}$ or go back to the "G" state with a probability $1-\mathrm{P}_{T_{n+1}}$, depending on whether the received SNR goes below a certain threshold value or not [34].

When a remote encounters a fade in a given cycle of the multiple access scheme (a TDMA frame in our case) it loses data in that cycle. There is a high probability that the channel might not recover from this impairment in the following cycle. This is owing to the fact that both shadowing and fading are slow processes compared to the data rate. Hence, a scheme with a fast reaction to these channel impairments must be devised in order to increase the utility of the wireless link. These combatting techniques affect the distribution of ON and OFF state intervals.

a) Distribution of fade/nonfade durations

In a BIWN system, fading can be characterized by slowly varying, frequency selective, Rayleigh fading [34], [36]. It can be shown that the instantaneous received power $\left[P_{r}=(1 / 2) r^{2}\right.$, where $r=$ amplitude of the signal at the receiver $]$ has an exponential distribution given by:

$$
f_{P_{r}}\left(P_{r}\right)=\frac{1}{\sigma^{2}} e^{\frac{P_{r}}{\sigma^{2}}}
$$

This leads to the fact that power reception at the receiver is a memoryless process. Values assumed by $P_{r}$ at the receiver determine the fade/non-fade intervals (durations). Thus the probability of $P_{\mathrm{r}}$ assuming a certain value in the present state doesn't depend on the past 
history of the process. This implies that the probability of $P_{r}$ dropping below a certain threshold (fade durations) is independent of the previous distributions. Thus the non-fade durations are also exponentially distributed and its probability density function can be expressed as follows:

$$
f_{\tau_{N F}}\left(\tau_{N F}\right)=\frac{1}{\bar{\tau}_{N F}} e^{\frac{\tau_{N F}}{\bar{\tau}_{N F}}}
$$

where the $\bar{\tau}_{N F}$ is the mean non-fading duration [36]. A deep fade in an indoor channel can last for a period ranging from a few milli-seconds to several seconds, or even minutes [34]. The methods employed to regain a faded signal (to prevent a long period fade from bringing about an unacceptable loss of transmitted cells) determine the fading duration characteristics. At times, due to the signal being under a deep fade, the receiver is unable to retrieve the transmitted cells. Under such circumstances, the transmitter should be notified of the channel condition. It can thus be assumed, for such a system that fading approximately has a fixed period and that the channel can be represented by a fixed number of 'OFF' states in the channel model discussed earlier.

\section{b) Interference}

The bandwidth required to support multimedia traffic is quite high and thus we assume a small frequency reuse factor, e.g. 3 or 4 . This is a cause of severe co-channel interference. Further, there is also adjacent channel interference (ACD) arising from imperfect filtering. The interference characteristics depend on the nature of the cell traffic and the behaviour of the radio channel. Bursty traffic can be modelled as an aggregate of the traffics generated by a number of ON-OFF mini sources with exponential distributions for $\mathrm{ON}$ and OFF periods. Thus, it is reasonable to model interference as occurring in exponentially distributed bursts. 


\section{Chapter 4 \\ System Description}

The following are the principal issues in the context of Broadband Indoor Wireless Networks:

1. A mobile radio link is hindered by a number of propagation mechanisms (as discussed earlier in Chapter three), namely multipath scattering from objects near the mobile antenna, shadowing (obscuring the line of sight between the transmitter and receiver) by dominant obstacles, the propagation path losses and interference.

2. A form of dynamic bandwidth allocation scheme to manage bandwidth among active users has to be implemented. The broadband indoor wireless links accommodate a wide range of multimedia traffic, the user density within a building being much higher than outdoor. Consequently, employment of an efficient bandwidth management protocol is of immense importance.

To support multimedia services in BIWNs, the multiple access protocol should be based on the up-to-date information collected from the connected terminals. A polling scheme, modified to comply with wireless network necessities, seems to be capable to fulfill above requirements. It offers the capability of supporting "bandwidth on demand" mode, with full control over the procedure. The controller can follow the sudden changes in the bit- 
rate of the heterogeneous sources.

\subsection{System Description}

A typical diagram of the system is shown in Figure 4-1. Floor areas are divided into small radio cells called microcells. Each microcell is served by a single base station. The stations are connected to a broadband ATM wired network by an ATM switch. A limited number of terminals, capable of generating multimedia traffic (voice, video and data) is considered in each cell. We assume that the remotes communicate only with their home base station. A single cell scenario is considered with interference also being taken into account. The base stations and portables are equipped with sectorized antennas to combat co-channel interference. For the duration of a communication session, the portable and the base use a particular combination of sectors that may be changed under circumstances when the propagation environment changes or a new co-channel interferer comes on line. The Asynchronous Transfer Mode (ATM) standards have been considered as the basis of the transport protocols because it is expected to offer higher bit rate flexibility and a greater degree of integration, in both transmission and switching functions, than the Synchronous Transfer Mode (STM). Wireless user terminals transmit and receive traffic carried in ATM cells, the cell being the basic unit of transmission and consisting of 53 bytes ( 8 bits/byte).

A framed, time division multiple access scheme based on polling has been chosen. The polling approach has the appeal of simplicity and is justified by the fact that the number of terminals within a cell is expected to be small. Based on polling, the system adapts to sudden changes in the rate of ATM cells to any portable by assigning more or fewer slots per frame. Polling provides updated information to the base station about the users' buffer contents, thereby allowing the base station to exercise dynamic bandwidth allocation. Time slots are allocated to users in a cyclic fashion, within a TDMA frame. 


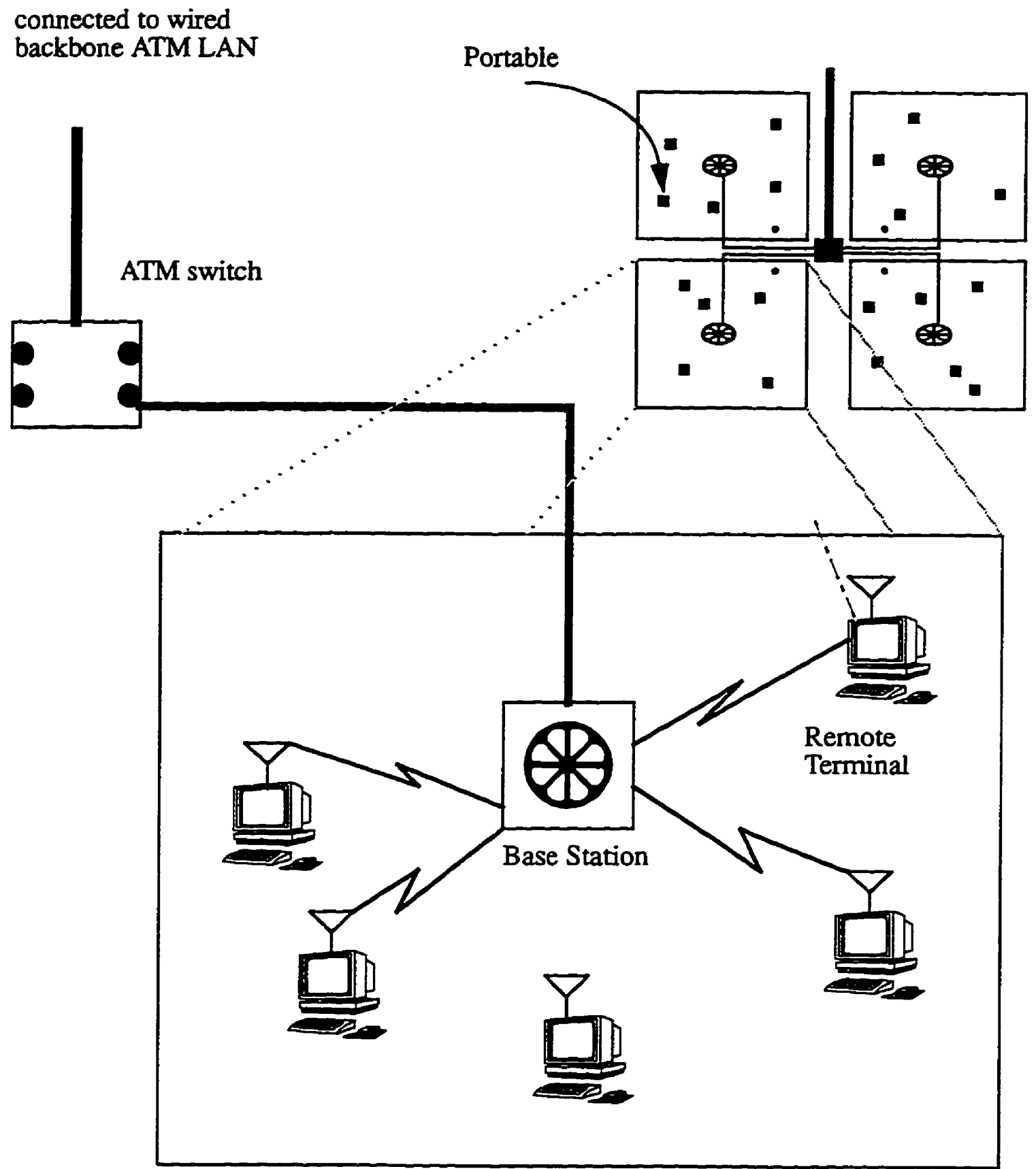

A Radio Cell

Figure 4-1: System Diagram 


\subsubsection{Frame Format}

Uplink (mobile to base) and downlink (base to mobile) traffic is carried in ATM cells, which are encapsulated along with overhead information, into envelopes for radio transmission. A time division duplex (TDD) mode is used, with uplink and downlink traffic and control envelopes occupying separate time slots in fixed length frames.

Traffic in each domain, or microcell, is organized by the domain's base station into frames of fixed duration, each consisting of both uplink and downlink traffic. The overall frame structure is shown in Figure 4-2. Each frame is comprised of two sections: 1) a short portion at the beginning of every frame which is called the control section, and 2) another section which contains the data cells called the data section. The first few slots in the control section are used for radio signalling, channel maintenance, pilot and beacon signals, etc. This is followed by a short period allocated for random access by new users, the slots being called registration slots. This period is marked by a special pilot signal. Receiving the pilot, a new user sends the connection request to the base station. Such requests could come from previously unregistered portables, or from portables currently homing on a different base station and/or frequency, who wish a hand off to this frequency/base station/sector combination. It is expected that in most cases the number of registration slots will be small (perhaps as low as one), since new registrations and handoffs are expected to occur relatively infrequently compared to the frame rate [37]. The data section has three parts. The first part contains the downstream traffic, during which the base station forwards the incoming traffic to remotes within the radio cell. Following the downstream part the base station starts to poll those remotes which should be served during the polling cycle. Each polled remote has to specify the number of slots it needs to empty its buffer during the service time. If the order of polling is fixed, the first polled queue gets serviced right after the polling duration in a TDMA cycle, while the $\mathrm{j}^{\text {th }}$ 


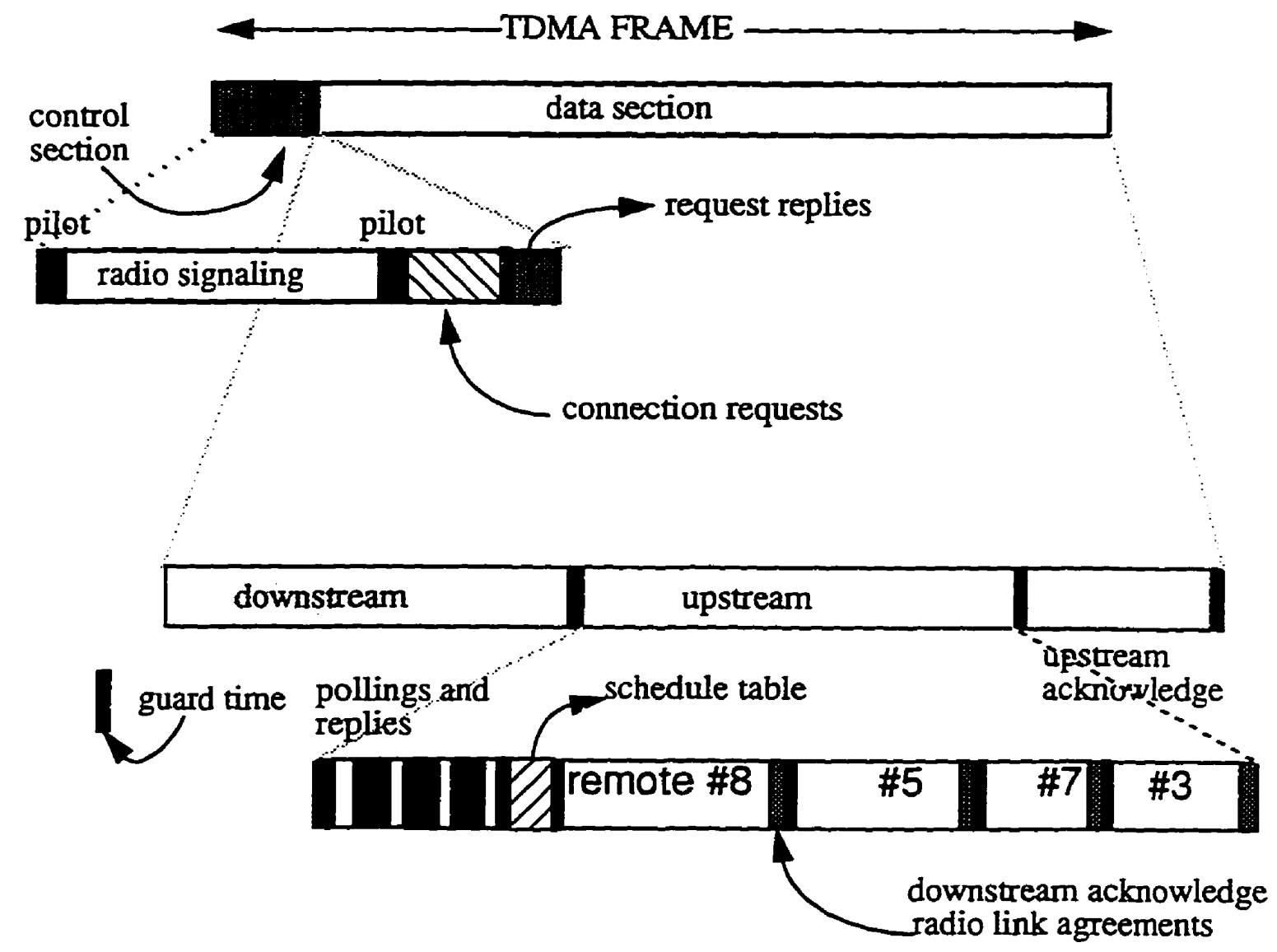

Figure 4.2 The structure of a TDMA frame

Figure 4-2: The structure of a TDMA frame 
queue has to wait till the service of the previous $(j-1)$ queues is completed. Thus, depending on how later a remote is served, there would be a longer delay between its polling and service instants.To provide equal condition for all the remotes, one may change the polling order in every cycle, so that every user has equal chance to get the first position. In the radio environment, randomly changing the polling order is beneficial in terms of randomizing the interference happenings among the remotes. Suppose that a strong signal from another nearby area cell, with the same frequency band is interfering into the communications in the concerned area cell. Since the base stations are assumed to be synchronized, the interference is likely to occur frequently in certain slots. In such a situation, if the polling order is fixed, the probability of having erroneous slots is higher for one of the remotes. Randomizing the polling order shares the interference probability among all the users. Finishing the polling, the base broadcasts a schedule table containing the name, the order of service and the number of allocated slots to the remotes which are going to be served during the data section. Each remote which finds its name in the schedule table occupies the slots in the upstream part (remotes to base) which are allocated to it, based on the order and the number of cells specified in the table. At the end of service time, each remote acknowledges the base station that it has received the downstream cells and requests the repeat if any cell is missing or erroneous. The radio link agreements (such as switching to another pair of antennas in antenna diversity) can be performed in this period as well. After the upstream part, the controller broadcasts the acknowledgment of the cells received from the remotes and requests the repeat if any cell is missing or erroneous.

In the following sections, we briefly explain the characteristics of the supported multimedia services, their requirements, and the modeling methods. 


\subsection{Multimedia Services and Their Requirements}

The supported services in a multimedia terminal can in general be classified into two basic categories: the real-time traffic (RTT), and the non-real-time traffic (NRTT). The basic difference between the two is that in RTT the receiver at the end of the path uses the signal immediately, thus, a long delay is not tolerated. The following sections talk about other aspects of these two kinds of traffic.

\subsubsection{Real-Time Services}

They are characterized by small maximum transfer delay (MTD) requirement. Each transmitted cell in RTT has a time stamp, within which if a cell is not delivered to its destination, the cell won't be useful anymore and has to be dropped. From the bit-rate variations point of view, the RTT sources may be of constant bit-rate (CBR) type, or variable bit-rate (VBR) type. The CBR sources generate a periodic stream of cells during a call, so that the bit-rate is constant, and the inter-cell interval is fixed, determined by the bit-rate. The bit-rate in a VBR source, changes with time, and most likely has bursty characteristics.

The VBR sources may be:

- Sources such as voice with silence detection, which alternate between active period, emitting cells at a constant rate, and silence period with no transmissions. These sources can be modelled using a single ON/OFF Markov process [38].

- Variable rate cell stream, such as output of VBR video coder, whose instantaneous rate changes rapidly as the content of the filmed scene changes.

The VBR mode for compressed video supported by ATM is of particular interest because of a number of potential technological benefits. The VBR is expected to have an edge over 
the CBR both from the point of view of the network and the users' interests. However, VBR video is more sensitive to cell loss. Also, the traffic management for CBR connections is much easier than VBR ones. Both VBR and CBR services may be requested during a multimedia call. The modeling of CBR sources is a trivial issue, but VBR video modeling is a subject of extensive studies, which shall be presented briefiy in the following section.

\subsubsection{Modeling of VBR Video Traffic}

For bursty sources, such as VBR video, there is high correlation between consecutive arrivals. Several models have been proposed for VBR video traffic. A group of models use ARMA (auto-regressive moving average) process to describe VBR traffic. A simple model of this type uses a first order AR (auto-regressive) process with a Gaussian input to model the number of cells generated per frame of VBR video [39], [40]. This model is proposed on the basis that the frame to frame bit-rate has a bell-shaped histogram, which can be approximated by Gaussian density function. The coefficients of the AR process are determined by matching the average and autocovariance functions of the model and experimental data. Another method used for modeling VBR video traffic for simulation purposes is the TES (transform-expand-sample) method [41], [42]. The model applies two random sequences. The first one which consists of samples of a uniform random variable (RV) in $(0,1)$ interval, acts as the background sequence, and is used to model the first moment of the video traffic. The second one, which can be a general RV, combined with the first sequence, smoothed and filtered out, models the second moment of the video traffic. This technique can be utilized to model the VBR video traffic, at the block level as well as the frame level.

VBR Video Modeling for Analysis Purposes:

The second group of models, which are more useful for analytical works, estimates the 
aggregate video traffic by a renewal process for the inter-arrival times [43], or multi stage birth and death process for the bit-rate [39], [44], [45], [46]. With a detailed Markov Chain (enough large number of states) these models perform very well and are good models for traffic studies [47]. In [39], the model is a one dimension multi stage birth and death process with a fixed bit-rate in each state. This model doesn't consider the effect of scene changes. To capture both, the short term and long term correlations, two dimension Markov chain is proposed. One dimension corresponds to the short term correlation and the second dimension, with bigger jumps in bit-rate, corresponds to scene changes [44]. In general, an n stage birth and death Markov chain, can be considered as the superposition of n ON-OFF, two states, Markov mini-sources [48], [49]. In the model proposed by [46], the cell arrival process from a single video source is modelled as discrete state continuous time markov process with batch arrivals representing scene changes. The interval times between scene changes (batches) are assumed to be exponentially distributed, and the batch size is assumed to be constant.

\subsubsection{Non-Real-Time Services}

In contrast to the RTT, the NRTT is more tolerable to delay, but usually has more stringent cell loss probability requirement. The NRTT may be modelled by Poisson or Bemoulli process, when the data is packetized and sent through the link without correlation between inter-arrival times. For applications such as retrieval of document or still images for which cells are produced and transferred in bursts, sources may be modelled by interrupted (Markov modulated) Bernoulli processes (IBP or MMBP). The Markov process consists of two states, an active state and an idle state. The duration of each state is geometrically distributed with different parameters. The process remains in the active (idle) state with probability $p(q)$, and changes to alternative state with probability $1-p(1-q)$. Arrivals occur in a Bernoulli fashion (with a probability a) during the active state, and no cell is produced during the idle state. The maximum burstiness is achieved when every slot contains a cell 
during the active period, which is in fact the ON-OFF (birth and death) Markov Process [50]. The aggregate traffic of several data sources may be modelled by Markov modulated Poisson process (MMPP).

\subsubsection{Multimedia Traffic Modeling}

We conclude from our previous discussions that all kinds of ATM multimedia sources can be modeled by the superposition of a proper number of identical ON/OFF Markov minisources (Figure 4-3). The ON and OFF periods of a mini-source are geometrically distributed, where during the ON state, it generates ATM cells at its peak rate (PR) constantly. By superposition, we imply that the total number of ATM cells generated by the multimedia source over any given time-interval equals the sum total of the ATM cells generated by each individual mini-source during that time-interval. The percentage of the time that a mini-source is in ON state is termed the activity ratio (AR). The number of mini-sources $\left(\mathrm{N}_{\mathrm{s}}\right)$, the averages of $\mathrm{ON}$ and $\mathrm{OFF}$ periods $\left(\mathrm{T}_{\mathrm{ON}}\right.$ and $\left.\mathrm{T}_{\mathrm{OFF}}\right)$, and the bit-rate during the ON state, are important parameters and have to be determined carefully according to the actual traffic parameters. In Figure 4-3, $\mu(\mathrm{n})$ represents the cell arrival rate of the overall source at time slot ' $n$ '.

In [51], typical values for the model parameters of some multimedia sources are given. e.g. A voice call with silence detection, can be modelled by:

$$
\begin{aligned}
& N_{s}=1 \text { mini-source, with } P R=64 \text { kbps or } 32 \mathrm{kbps}, \\
& A R=0.4 \text { to } 0.5(0.35 \text { for } A D P C M \text { voice }), \\
& T_{O N}=1.2 \sec (.35 \mathrm{sec} \text { for ADPCM voice }) .
\end{aligned}
$$

Video conferencing with 1 Mbps peak-rate, can be modelled with the following parameters: 


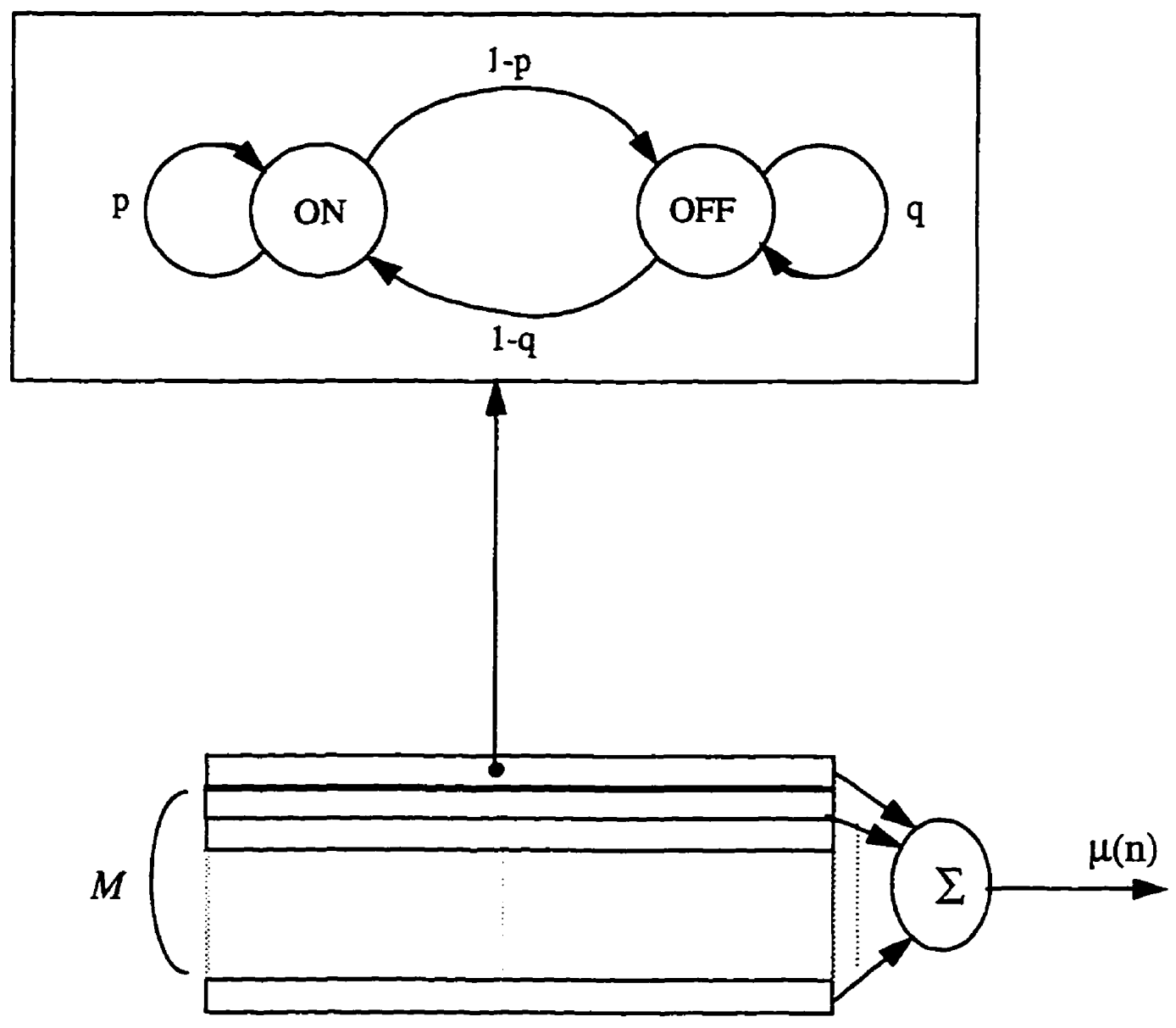

Figure 4-3: The ON-OFF Markov mini-sources model for VBR trafic 
$\mathrm{N}_{\mathrm{s}}=15$ mini-sources, with $\mathrm{PR}=66.7 \mathrm{kbps}$,

$\mathrm{AR}=0.32$, and $\mathrm{T}_{\mathrm{ON}}=0.033 \mathrm{sec}$

\subsection{Assumptions}

We consider only one radio cell, which is served by a single base station connected to a wired backbone broadband local area network. The synchronization between the base station and remote terminals is assumed to be perfect. During this study, only the uplink traffic (from terminals to the base) is considered. The ARQ is applied in which the erroneous cells are retransmitted in the next cycle. We assume that the time is slotted to ATM cell size. Each source is modelled as a set of identical and independent ON-OFF mini-processes, and the arrival process is the superposition of the output of $n$ independent sources. The system is studied under fixed configuration of connected sources, in the sense that there is no connection establishment or termination. Assuming stationarity, we study the system in the steady state conditions.

\subsubsection{Arrival Process Characteristics}

The cell arrival process of each source is assumed to be the superposition of a proper number of independent ON-OFF mini-sources as shown in Figure 4-3. If a mini-source is in ON state, it remains there in the next slot with a probability $\mathrm{p}$, and switches to OFF state with a probability 1-p. A similar phenomenon occurs when the mini-source is in OFF state (with probability q). Thus the process can be described by the following transition matrix:

$T=\left[\begin{array}{cc}p & 1-p \\ 1-q & q\end{array}\right]$

and the probabilities of being in each of the states, under steady state, are given by:

$\pi_{O N}=\frac{1-q}{2-q-p}$ 
and $\pi_{O F F}=\frac{1-p}{2-q-p}$

The mean of the process, which is the probability of producing a cell in a time slot is equal to: $\bar{\varepsilon}=\frac{(1-q)}{2-q-p} \bullet \gamma$

where $\gamma$ is the cell-rate during the ON state.

\subsection{Modeling of Self-Similar Variable Bit Rate Compressed Video}

Due to the advantages of VBR video transmission and the packet-switched nature of ATM, and given the development of highly-sophisticated compression techniques for video sources, VBR compressed video traffic is expected to become one of the main loading components in future broadband networks. However, the high bandwidth and burstiness of VBR video traffic, can make network design and management difficult to perform. Effective design and performance analysis depend on accurate modeling of the various traffic types. Traditional models based on Markovian structures (e.g., MMBP, IBP, etc.) have been widely used to statistically approximate VBR video traffic. These models share an asymptotically exponential decay of the auto-correlation function and a rapidly decaying marginal distribution tail. Furthermore, they lack a systematic way of simultaneously fitting both the empirical marginal distribution and the autocorrelation function.

Earlier efforts in modeling video traffic have been confined to short traces of empirical records or to conference video, due to the difficulties in obtaining empirical data from realistically long sequences. Recent extensive measurements of real traffic data [53] have led to the conclusion that VBR video traffic cannot be sufficiently represented by traditional models and that VBR video traffic possesses self-similar (or fractal) characteristics, meaning that the dependence in the traffic stream lasts much longer than 
traditional models can capture [54], [55].

The crucial feature of self-similar processes is that they exhibit long range dependence (LRD), that is, their autocorrelation function $r(k)$ decays less than exponentially fast, and is non-summable, i.e., $r(k) \sim k^{-\beta}$, as $k \rightarrow \infty$, for $0<\beta \leq 1$ (the quantity $H=1-\beta / 2$ is called the Hurst parameter). This is in contrast to traditional stochastic models, all of which exhibit short range dependence (SRD), i.e., have an autocorrelation function that decays exponentially or faster. For formal definitions of self-similarity, second-order selfsimilarity, and asymptotical second-order self-similarity the interested reader can see [55] and references therein.

There are numerous stochastic models which exhibit the self-similar property, two of them, namely the exactly self-similar fractional Gaussian noise (FGN) and the asymptotically self-similar fractional autoregressive integrated moving-average (FARIMA) process, are the most commonly used. Hosking's procedure [56] was used to generate traces from FGN. This method is applicable to any Gaussian process as long as the correlation function $r(k)$ is known. An example of the typical autocorrelation structure of MPEG video, at the frame level, is shown in Figure 4-4. In order to simulate the oscillations in the correlation function, composite models are typically used, i.e., models based on combining three different processes with marginal and correlation structures matched to the I,B, and P empirical records, respectively. The goal is to generate a process with marginal distribution and autocorrelation function that closely match the corresponding functions of empirical traces. The approach is briefly summarized as follows [57]:

(1) Measure the Hurst parameter of the empirical data or decide on a Hurst parameter if measurements are not available. 


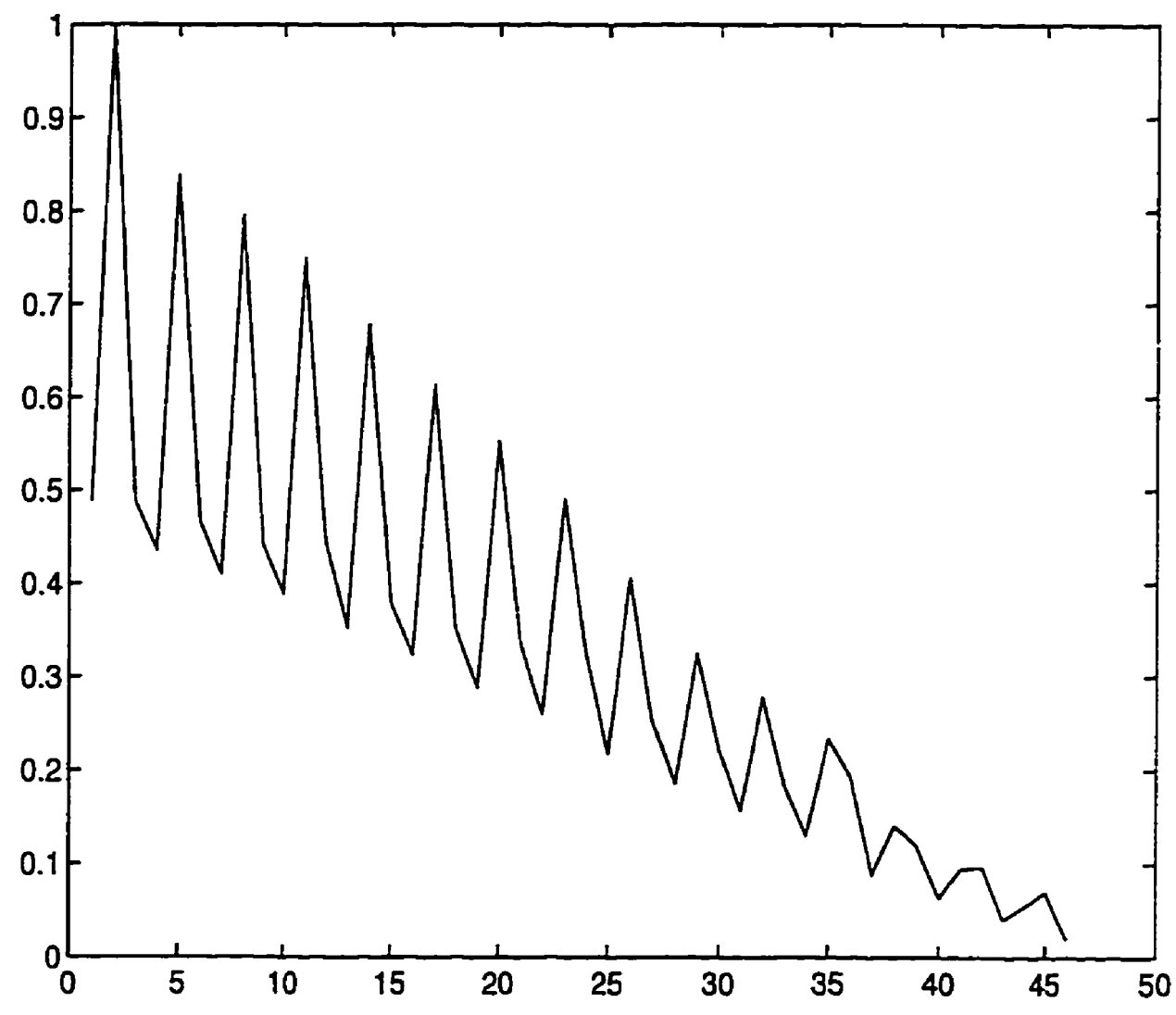

Figure 4-4: Autocorrelation of bytes per frame in VBR MPEG video 
(2) Generate a single background FGN process by using Hosking's technique.

(3) Generate the foreground I, B, P sequences by using appropriate histograms.

(4) Interactively fit the autocorrelation to the empirical records by fine tuning weights incorporated in our model to match the short term correlation.

In [57], approximately two hours of video from the movie "Last Action Hero" was used. The movie was initially encoded using the MPEG-1 algorithm [58], [59], with a hardware intraframe MPEG-1 encoder on a Sun SPARC 20 computer [60]. The movie was then decompressed and re-encoded with both intraframe and interframe coding, using the PVRG-MPEG 1.1 software codec [61]. 


\section{Chapter 5 \\ Simulation Technique and \\ Discussions}

This chapter deals with the description of the simulation approach used to evaluate the performance of an indoor wireless network with an emphasis on VBR video traffic and the discussion of the results obtained using the simulation tool.

Simulation was carried out with VBR video data derived using two methods:

a) Video data generated from ON/OFF Markov mini-sources model as discussed in Section 4.2.4, with proper parameteric values.

b) Variable bit rate compressed video data obtained from models exhibiting self-similar traffic characteristics.

For both the sources ( $a$ and $b$ ), the simulation was carried out with emphasis on steady state performance, as indicated by cell loss probability (CLP), average waiting time of cells and mean buffer occupancy for a given user. These results are dependent upon the following simulation parameters:

1) The nature of the video traffic (burstiness) generated by the given user. 
2) The minimum guaranteed bandwidth for the given user.

3) The channel model parameteric values (transition probabilities).

4) The maximum transfer delay (MTD) for the given user data.

Results have been obtained, in which we varied one of the above mentioned parameters and keeping the other parameters fixed.

\subsection{Description of Simulation: 'The Single Terminal}

\section{Approach'}

The aim of the simulation is to determine the performance of a given terminal in a multiple access network that serves heterogeneous traffic. We focus on the variable bit rate (VBR) video traffic. We consider a single cell scenario in a BIWN, with a single base station serving this cell.

- A limited number of statistically identical terminals, generating VBR video traffic, is considered. These scattered portables of the given cell transmit and receive traffic carried in ATM cells, the cell being the basic unit of transferred data.

- A TDMA scheme based on polling has been chosen to allocate time slots to the connected remotes, within a multiple access frame. By polling the users, the base station utilizes the updated information about the users' buffer contents to carry out statistical multiplexing.

- We focus on the transmission (uplink) of a single specific terminal. The traffic generated by this terminal is modeled by a simulated video source, explained later in the chapter. The buffer contents of only this terminal are tracked with the progress of the simulation. 
- The aggregate of the uplink traffic generated by the 'other terminals' and the downlink traffic (from the base station to the portables) in the cell is modeled by the sum total of the outputs of the simulated VBR video sources.

The transmissions of the other terminals in the cell are not considered separately. Thus, the variation in the buffer contents of the rest of the terminals in the cell is not followed separately in the simulation. This approach results in a simplified simulation technique.

The simulation starts at time ' $t$ ' $=0$, when transmission from all the terminals in the given cell begins. All terminal buffers are assumed to be empty at this instant. The ATM cells ( 53 bytes per frame) are generated every video frame duration ( $33 \mathrm{msec}$ in our case) by the terminals.

The specific terminal under consideration transmits the first batch of video cells which get stacked in its buffer. Similarly, video cells generated by other terminals also get stacked in their respective buffers.

Individual consideration to the buffer contents of the other terminals is not given. The sum total of all these buffer contents and the downlink traffic in the cell is represented by the sum total of the traffic generated by a number of simulated video sources, explained later in the chapter. This traffic constitutes a portion of the block labeled 'the rest of the system' in Figure 5-1 and is represented by the block labeled 'other sources' in Figure 5-2 and Figure 5-4. This traffic competes with the traffic generated by the specific terminal under consideration, for time slots within a multiple access frame.

The buffer contents of a given terminal may be represented by the following equation:

$$
T(n+1)=T(n)+A(n)-W(n)
$$


$T(n)=$ Buffer Contents of the given terminal just before transmission of cells in the $n$th multiple access frame.

$A(n)=$ New Arrivals in the terminal buffer in the $n^{\text {th }}$ frame.

$W(n)=$ Number of time slots allocated to the terminal in the $n^{\text {th }}$ frame.

This simulation approach, where only the transmission of a specific terminal is monitored through the simulation run, is termed as the 'single terminal approach'. Figure 5-1 depicts the system as viewed by the given terminal. In the model, cells pass through a cascade of the terminal buffer, the multiple access time window and the channel status. A queueing model was applied to the terminal buffer. The system effect shows up as a random channel time window.

\subsubsection{The Buffer management:}

The video traffic generated by a given user is organized in frames that occur at a rate of one frame per $33 \mathrm{msec}$. The basic unit of transmission is an ATM cell. These transmission units are stacked in the terminal's buffer in batches, every video frame duration. A new cell is stamped with an expiry time. Cells in the buffer are ordered according to their expiry time. For real - time traffic (e.g.: video), this expiry time is also termed as the Maximum Transfer Delay (MTD). The cells are evaluated for their residency time in the queue. Expired cells, i.e. the cells whose residency times exceed the MTD are discarded.

\subsubsection{Multiple Access Time Window:}

Eqn. (5.1) expresses the contents of the terminal (number of ATM cells stacked in the terminal buffer) buffer in the $(n+1)^{\text {th }}$ frame, in terms of the contents of the buffer in the $\mathrm{n}^{\text {th }}$ frame, arrivals of ATM cells from the terminal source if any, and the number of time slots allocated to the terminal in the previous $\left(n^{\text {th }}\right)$ frame.

The first portion of the multiple access frame contains the downstream traffic, when the 


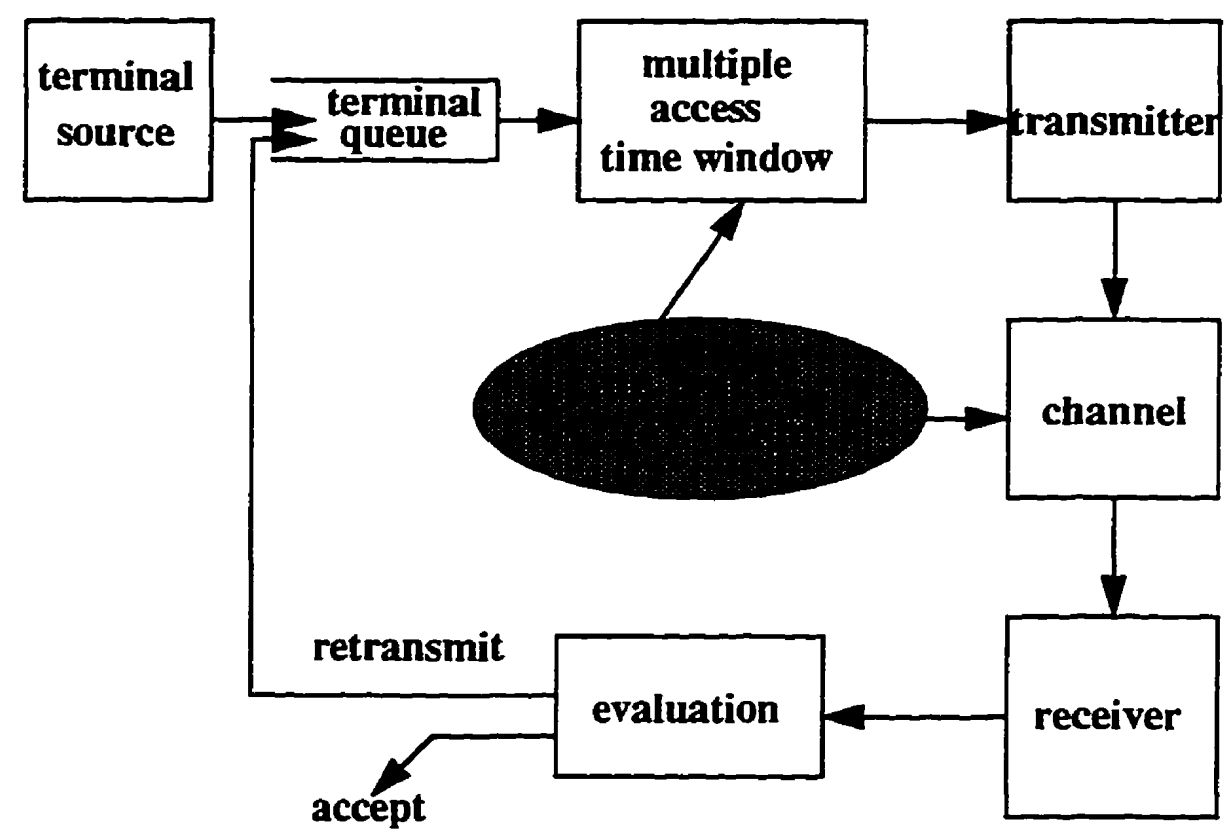

Figure 5-1: The System As Seen By A Given Terminal 
incoming traffic in the cell is forwarded to the remotes. Following the downstream portion, remotes are polled by the base station. Each polled remote specifies the number of slots it requires in the multiple access frame to empty its buffer. Let ' $T$ ' be the number of ATM cells present in the given terminal buffer at the instant of polling. The given terminal then requests ' $T$ ' time slots for transmission in that TDMA frame.

Let ' $C$ ' be the total number of time slots required by all other terminals in the uplink and the downlink. Let ' $N$ ' be the total number of traffic slots in a multiple access frame.

Each terminal is assigned a minimum guaranteed bandwidth by the controller, i.e. an interval of $\mathrm{T}_{\text {min }}$ (in ATM cell duration slots) in the fixed length frame. The controller assigns ' $W$ ' slots to the given terminal in the frame, based on the following algorithm:

Step I: Check if $\mathrm{T}>\mathrm{T}_{\mathrm{min}}$. If yes, go to step III. Else go to step II

The controller checks if the number of time slots requested by the portable is greater than the minimum assigned bandwidth.

Step II: $\mathrm{T}<\mathrm{T}_{\min } ;$ thus $\mathrm{W}=\mathrm{T}$.

In case the buffer contents of the terminal is lower than the minimum guaranteed bandwidth, the terminal gets whatever it has demanded.

Step III: If $(T+C)>N$, then $T_{\min }<W<T$. Else $W=T$.

If the sum of the demands of the given terminal and the 'other sources' exceeds the total number of data slots in the multiple access frame, then the given terminal is assigned $\mathrm{W}$ slots for transmission, which is uniformly distributed in the interval $\left[T_{\min }, T\right]$. Otherwise, the terminal gets whatever number of slots it has requested.

Time slots are allocated to the given terminal in every multiple access frame using the above algorithm. This time frame has been called multiple access time window. The algorithm for allocation of time slots is represented in Figure 5-2. 


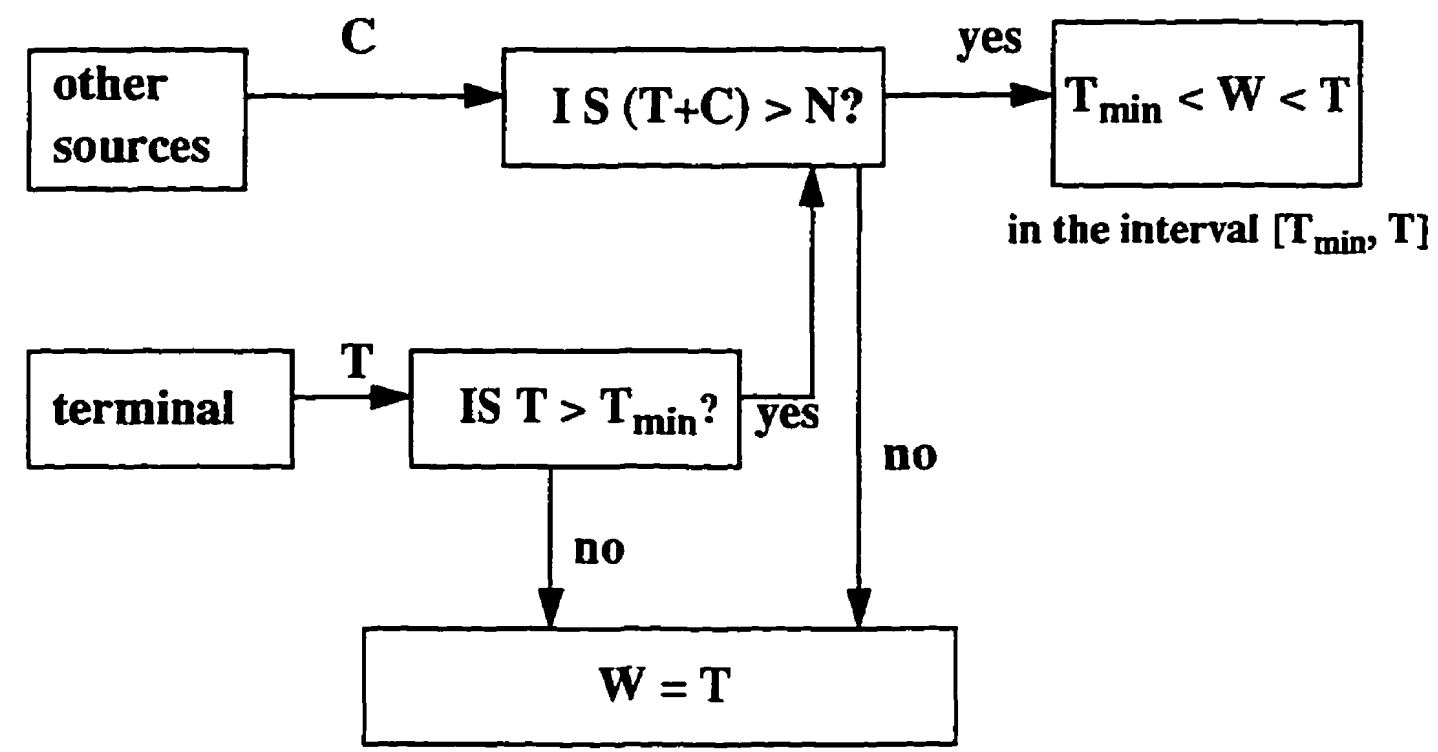

$\mathbf{W}=$ Time assigned to a terminal in a frame

$T=$ Time requested by the terminal

$\mathrm{C}=$ Time requested by all other sources (uplink transmission from other portables

$\mathbf{N}=$ Frame duration + downlink traffic)

$T_{\min }=$ Minimum Guaranteed Interval assigned to a terminal in a time frame

Figure 5-2: The Multiple Access Channel Window 


\subsubsection{The Channel Block:}

A transmitted radio signal in an indoor wireless system suffers from fading, shadowing and interference. The channel block subjects the transmitted cells to random errors in accordance with the link conditions. In that sense, the channel block is a random on/off process.

It was suggested in [1],[36] that the indoor channel behaviour can be approximated by a fade/non-fade (ON/OFF) model with exponentially distributed ON and OFF states. We propose a similar ON/OFF channel model which considers the effects of fading, shadowing and interference separately (discussed previously in Section 3.3), as shown in Figure 5-3.

The model takes into account the fact that the distribution of the ON and OFF state intervals of the ON/OFF model is a function of the effectiveness of the techniques employed to combat fading, shadowing and interference. We further assume that the schemes employed to mitigate the above mentioned channel impairments shall be effective enough to recover the channel from a long shadowing condition in at most three states, and from a fade in two states and from an interference condition in one state. Each of these states corresponds to one TDMA frame time. Transition Probabilities I, II,... X. must be defined for a simulation run.

\subsubsection{The VBR Video Traffic Source:}

VBR Video traffic has been generated using two techniques:

1) Modelled by the superposition of a proper number of identical ON/OFF Markov minisources, discussed earlier in 4.2.4. The ON and OFF periods of a mini-source are 


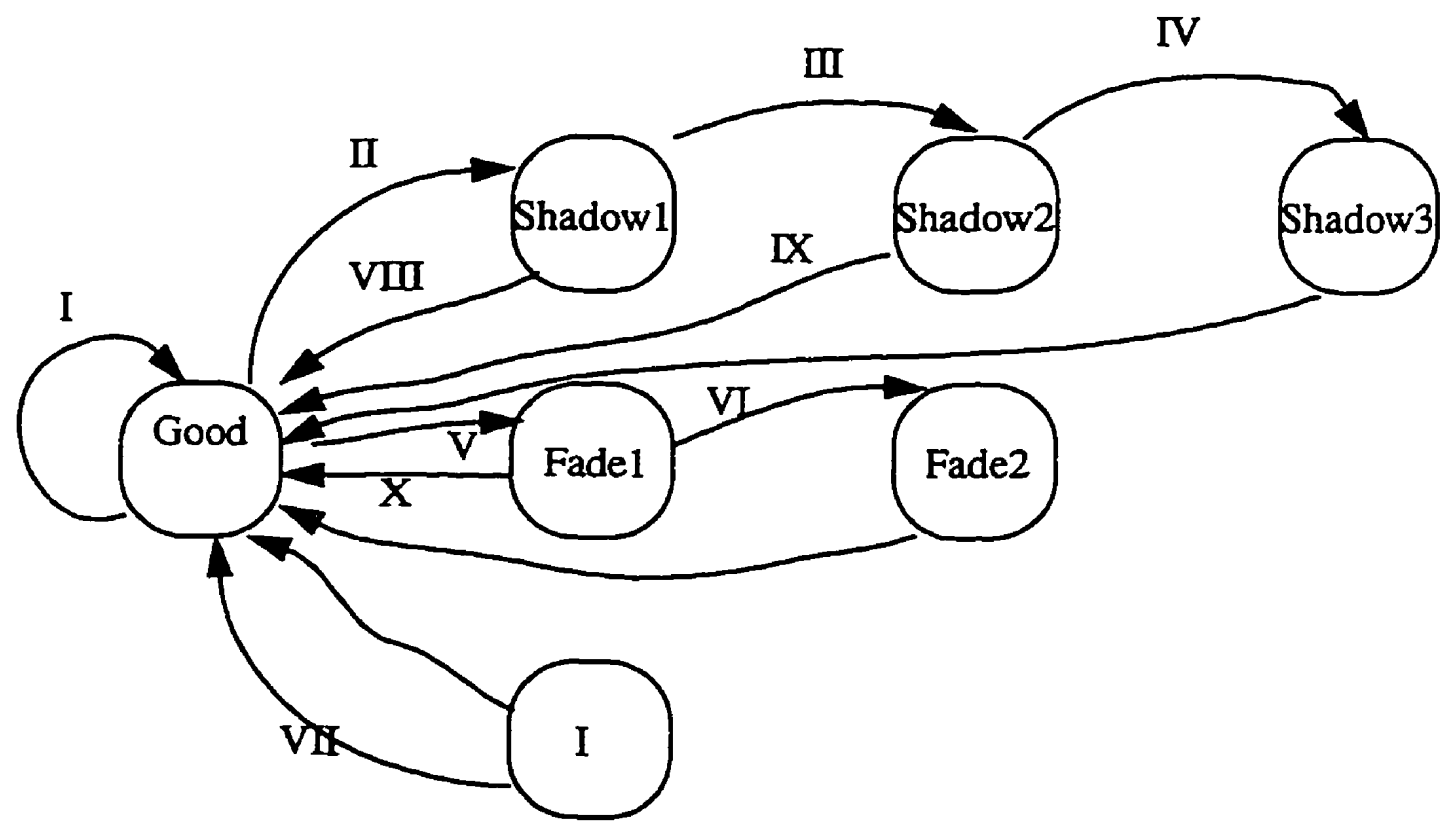

Figure 5-3: The Channel Model 
geometrically distributed, where during the ON state, it generates ATM cells at its peak rate (PR) constantly. The number of mini-sources $\left(\mathrm{N}_{\mathrm{s}}\right)$, the averages of $\mathrm{ON}$ and $\mathrm{OFF}$ periods ( $T_{0 n}$ and $T_{o f f}$ ), and the bit rate during the $O N$ state, are important parameters and their proper values have to be chosen carefully.

2) VBR compressed video data derived from models exhibiting self-similar traffic characteristics (described in Section 4.4). This data is available in the form of I, B and P frames.

The above techniques were used to generate VBR Video data with a wide range of burstiness characteristics. The ON/OFF model consisting of Markov mini-sources (technique 1 stated above) was used with varying parameters, viz. $\mathrm{N}_{s}, P R, A R$ and $T_{O N}$ (Ref. Section 4.2.4) to simulate sources generating bursty video traffic of varied nature. Also, using technique 2 stated above, different I, B and P sequences were used to generate data with heterogeneous traffic characteristics. Based on the above methods, a sufficiently large number of independently obtained data was used to simulate the overall bursty video traffic aniving at the base station (uplink and downlink) in the given radio cell. The varied nature of these simulated traffic sources (a sufficiently large number) is assumed to represent the actual overall traffic scenario at the base station in a micro-cell in a broadband indoor wireless system.

VBR Video traffic for the given user was also simulated using the above two approaches. This simulated traffic for the given user is transmitted on the uplink (portable to the base station). The given user competes for time slots within a TDMA frame with the other cell traffic (uplink and downlink traffic) as shown in Figure 5-2.

\subsubsection{Multiple Access Scheme}

The length of the multiple access frame (TDMA frame) is constant. Although variable 
length frame could be more efficient, keeping the frame length constant simplifies the analysis and the implementation. The frame length has been chosen to satisfy the requirements of the video frame rate and the audio delay constraints. In our simulation we have assumed that the video frame rate is 33 frames per second. In other words, the duration of the multiple access frame should not exceed 1/33 sec. Thus the upper bound on the frame length is $33 \mathrm{msec}$. The lower bound on the frame length is set by the maximum allowable delay of the audio signal, which is about $2 \mathrm{msec}$. The local access portion of the end-end delay is minimal and has been assumed to be $2 \mathrm{msec}$. This results in a $2 \mathrm{msec}$ lower bound on the frame length. It is advantageous to choose the lower bound because such a choice will satisfy the audio delay requirements, and at the same time would allow a video frame to be transmitted over a number of channel frames. Spreading the video frame over several channel frames allows the terminal and base station to negotiate the required bandwidth more efficiently. In general, a short frame increases the overhead and reduces the overall efficiency. The choice of ine frame length is a compromise between delay and efficiency.

In [37], a dynamic TDMA polling based scheme has been proposed for an indoor broadband wireless LAN. In the same work, simulations have been performed choosing the multiple access frame to consist of 204 time slots. Out of these, 180 slots belong to the data section, while 24 belong to the control section (the concept of data and control sections has been discussed in section 4.1.1). For the purpose of consistency and for comparison of results to be possible we have assumed the same values, viz. the TDMA frame is assumed to be consisting of 204 time slots and the data and control sections are assumed to be consisting of 180 and 24 slots respectively. As stated earlier, each time slot is of 1 ATM cell duration. In other words, the number of ATM cells transmitted per frame is 204. The network capacity has been chosen to be $31.45 \mathrm{Mbps}$ for the purpose of convenience. This choice of the network capacity results in a multiple access frame of 
duration $2.75 \mathrm{msec}$, which places us between the lower bound and the upper bound on the frame duration, but closer to the lower bound. Each time slot corresponds to a duration of $13.48 \mu \mathrm{sec}$. Utilization may be defined as the total load offered in the upstream and the downstream divided by the total bandwidth allocated to both the upstream and downstream traffic (network capacity). The polling overhead has been neglected such that all the 180 slots in the data section can be assumed to be available for transmission of user data (to/from the base station). The simulation was performed for the period of transmission of 17000 video frames ( 1 frame is of $33 \mathrm{msec}$ duration) by the given user over the uplink. The terminals as well as the controller (base) are assumed to be synchronized. The network is assumed to be in steady state with no new connection establishment or connection termination. A selective repeat ARQ is assumed to be employed to recover the erroneous received cells from the given user.

\subsection{OPNET}

Simulations were carried out using the OPNET (OPtimized Network Engineering Tools) simulation package. It is a comprehensive engineering system capable of simulating large communications networks with detailed protocol modeling and performance analysis. OPNET features include:

- A graphical specification of models

- A dynamic, event-scheduled Simulation Kernel

- Integrated data analysis tools

- A hierarchical, object-based modeling

OPNET delivers open systems methodology and an advanced graphical user interface known as the MII 3 User Interface. The package is based on eight tools that fit into three 
categories as described below:

\subsubsection{Model Development:}

This consists of the Network, Node, Process and the Parameter Editors, which are the primary development tools. They each have the single purpose of defining models at one level of the modeling hierarchy. Typically, initial design efforts in a project will focus on developing node models for each distinct type of node. Process models will initially be place holders, but they will be successively refined during model development. The network model, consisting of nodes and links must be defined before the network simulation can be built. The Parameter Editor is used in conjunction with process and node model development to define special model structures.

\subsubsection{Simulation Execution:}

It consists of the Probe Editor and the Simulation Tool. This typically involves the preparatory step of attaching probes to the points of interest in the model using the Probe editor. Probes monitor the statistics computed during a simulation. The Simulation Tool allows specification of a sequence of simulations which use particular input and output options. It uses spreadsheet-like tables for data entry.

\subsubsection{Results Analysis:}

This consists of the Analysis Tool and the Filter Editor. The Analysis Tool presents statistics gathered during simulations in the form of two dimensional graphs or text listings. The information collected by a simulation can be viewed directly, or processed by filters. The Filter Editor is used to define filters to mathematically process, reduce, or combine statistical data.

The network model is the highest "domain" in OPNET"s hierarchical modeling structure. Depending on the type of the network being modeled, a network model may consist of 
communicating nodes connected by point to point links, bus links, radio links or just a single node. Nodes and links can be placed within subnetworks, which can then be treated as single objects in the network model. This is useful for compartmentalizing the network diagram, and provides a quick way of duplicating groups of nodes and links.

Using the Network Editor is often the last step in creating a network simulation, since node models and process models have to exist before network models containing them can be created. However, top-down design can be performed to a certain extent if node and process models are under-specified "stubs" which will be enhanced later.

The node models created are placed as node instances in networks via the Network Editor. OPNET node models are modular. A node is defined by connecting various module types with packet streams and statistic wires. The connections between modules allow for guided packet and status information exchange between modules. Each module placed in a node serves a unique purpose, such as generating packets, queueing packets, processing packets, or transmitting and receiving packets.

The Process Editor is used to construct the processes that run in processor and queue modules. At the core of most OPNET simulations are user-defined process models. Process models can represent the logic of communications hardware, network protocols, distributed algorithms, or high level client server processes. OPNET simplifies the construction of process models by offering graphical representation of extended finite state machines. Besides the graphical structure of the FSM diagram, process models contain textual specifications which describe the precise behaviour of the process. The combination of graphical and textual specifications forms an expression in Proto- $C$, the OPNET protocol language. The text portion of Proto- $C$ is a variant of the $C$ language with both limitations and enhancements. 
Figure 5.4 depicts the entire simulation procedure by combining various blocks explained in the earlier sections and presenting them as a whole simulation module. The specific terminal under consideration is represented by the block labeled 'source'. The source transmits video cells (ATM cells), with an expiry time stamped on each cell. These cells get stacked in the terminal buffer in the order of their expiry times. The expiry time of a cell has also been referred to as the Maximum Transfer Delay (MTD). The cell with the lowest expiry time is placed ahead of the rest in the queue and vice-versa. A delay check is carried out on the cells to arrange them in order of their expiry times. Expired cells are discarded. The following check is carried out to test if the expiry time has been exceeded.

Let $S=$ Simulation Time, $G=$ Cell Generation Time $=$ Time of arrival of a cell in the queue, and $R=$ Residency time of the cell in the queue.

The following relation holds: $R=S-G$. If ' $R$ ' is greater than the expiry time of the cell then the cell is discarded.

Cells ahead in the queue have a greater priority for transmission than those behind them. The terminal requests ' $T$ ' time slots in the multiple access frame, equal to the number of ATM cells present in its buffer.

In the block labeled 'time assignment', allocation of time slots to the given terminal is made, based on the time assignment algorithm described earlier in Section 5.1.2 and Figure 5-2. The sum of the collective demand (for time slots in the given Multiple Access frame) of all the other portables and the downlink traffic is represented by the arrow labeled 'load', which is the input to the 'time assignment block'.

The given terminal transmits ' $W$ ' cells from its buffer after the allocation of ' $W$ ' time slots in the given Multiple Access frame. Depending on whether the channel is in a good or a bad condition, these cells are either received successfully or a retransmission of those ' $W$ ' 
cells is requested. It has been assumed that the length of the transmission of the given terminal in a multiple access frame is small enough so that under conditions when the channel is in a fade or a shadow, either all the cells transmitted by the terminal in a multiple access frame reach the receiver successfully or all the cells are lost. On successful reception of the cells, an 'ACK' is sent to the transmitter (portable). A copy of the transmitted cells is kept in the terminal buffer till an 'ACK' is received. In case no 'ACK' is received, the cells are retained in the buffer for transmission in the next frame.

\subsection{Simulations with VBR Video sources based on ON/OFF Markov Mini-Sources Model}

In this section, we present the results of a set of simulations carried out with traffic sources modelled using the ON/OFF Markov Mini-Sources model.

In [44], a continuous time Markov chain model has been proposed to represent VBR video sources and it has been suggested that superposition of identical ON/OFF mini-sources may also be used to represent the VBR video source.

The VBR encoder output of a CATV source, has a bit-rate ranging from 0 to $44.1 \mathrm{Mbps}$, with an average at $16.8 \mathrm{Mbps}$ [52]. It can be modelled [51]by:

$$
\begin{aligned}
& N_{s}=30 \text { mini-sources, with } P R=1.47 \mathrm{Mbps}, \\
& A R=0.38 \text {, and } T_{O N}=0.033 \mathrm{sec} .
\end{aligned}
$$

The choice of $\mathrm{N}_{\mathrm{s}}$ comes from the maximum bit-rate of the CATV source ( $44.1 \mathrm{Mbps}$ ) and a choice of $P R=1.47 \mathrm{Mbps}$. By definition, $A R$ is the ratio of the average bit-rate to the peak bit-rate. 'AR' has been fixed to 0.38 , based on the ratio of $44.1 \mathrm{Mbps}$ and $16.8 \mathrm{Mbps}$. 


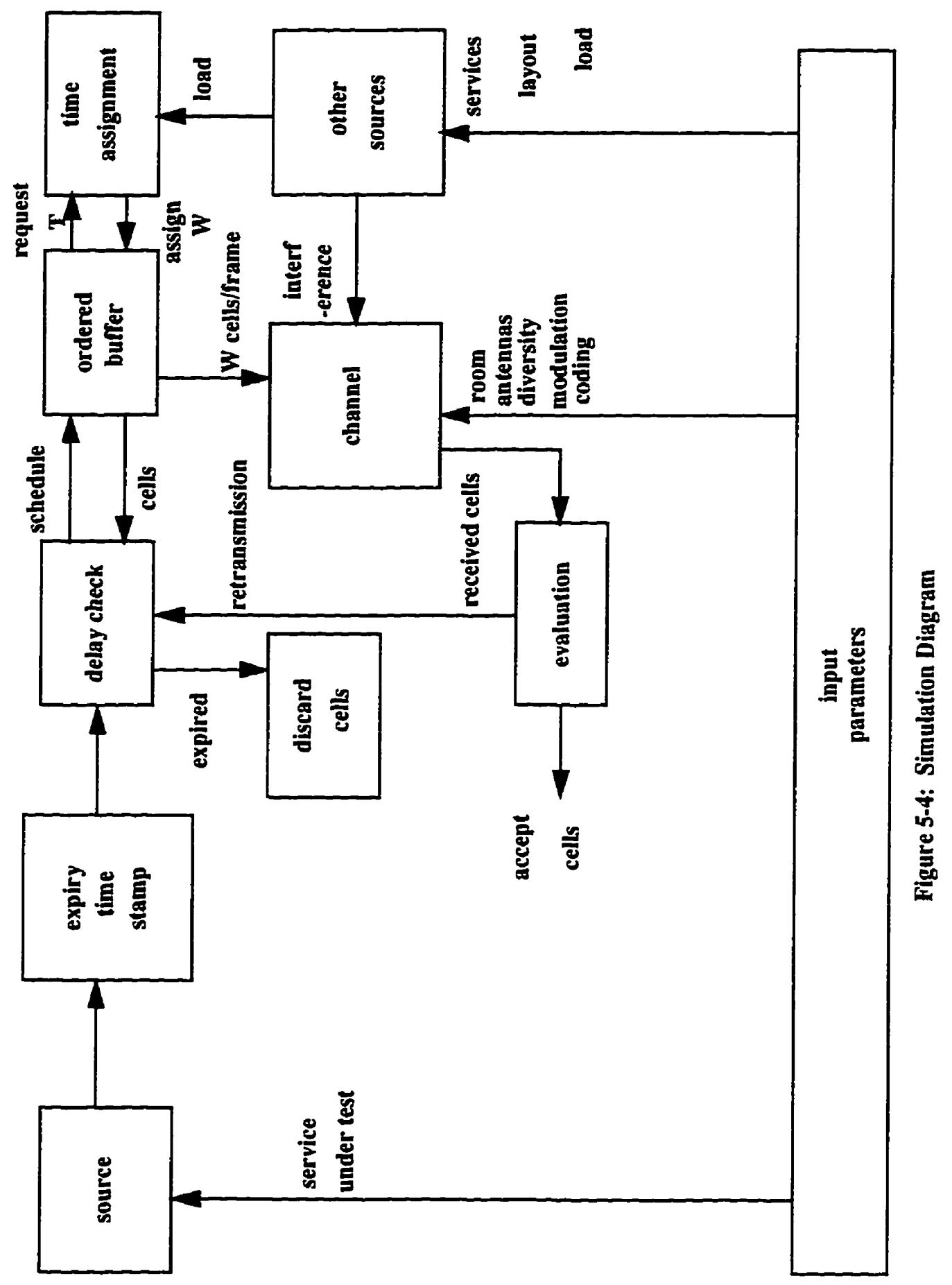


The choice of $T_{O N}$ is from the frame transmission rate of 30 per second.

All the video sources in our simulation were modelled using the ON/OFF Markov minisources model (Modeling technique described earlier) with the following characteristics:

The given video source (the 'given user') was modelled by $20 \mathrm{ON}-\mathrm{OFF}$ sources, with 0.375 activity ratio (AR), $0.033 \mathrm{sec}$ for mean $\mathrm{ON}$ duration, and $P R=0.27 \mathrm{Mbps}$. Similar to the modeling of the VBR encoder output explained earlier in this section, the above mentioned choice of the ON/OFF source parameters, viz.:

$\mathrm{N}_{\mathrm{s}}=20, \mathrm{PR}=0.27 \mathrm{Mbps}, \mathrm{AR}=0.375$, and $\mathrm{T}_{\mathrm{ON}}=0.033 \mathrm{sec}$, results in the modeling of an MPEG VBR Video Source [27] with a peak bit-rate of $5.5 \mathrm{Mbps}$ and a mean bit-rate (MBR) of $2.06 \mathrm{Mbps}$. The autocorrelation function of the traffic generated by the simulated video source is found to be similar to that of the MPEG VBR video data, with the choice of the model parameters, $N_{S}, A R, P R$ and $T_{O N}$.

The remaining sources ('other sources') were modelled with characteristics listed in Table 5-1. The mean ON duration for all the sources was $0.033 \mathrm{sec}$.

The values in the column labeled 'MBR' represent the Mean Bit-Rate of the traffic generated by the modeled video source, AR represents the activity-ratio of each minisource, and $\mathrm{N}_{\mathrm{s}}$ represents the number of mini-sources used. For e.g., the first source (MBR $=1.28, A R=0.32$, and $N_{S}=15$ ) was modeled using $N_{S}=15, A R=0.32, P R=0.267$ Mbps and $T_{O N}=33 \mathrm{msec}$. This is also found to model VBR MPEG video. The remaining values for the three parameters, viz. MBR, $A R$ and $N_{s}$ have been arrived at in the same manner. $\mathrm{N}_{\mathrm{s}}$ has been assumed to be 20 for most of the modeled sources as shown below. With this value of $\mathrm{N}_{s}$, the autocorrelation function of the generated VBR video traffic is found to match that of MPEG VBR video data. 
Table 5-1: Video Sources Based On On/Off Markov Mini-Sources Model

\begin{tabular}{|c|c|c|}
\hline $\begin{array}{c}\text { MBR } \\
\text { (Mbps) }\end{array}$ & AR & $\mathbf{N}_{\mathbf{s}}$ \\
\hline \hline 1.28 & 0.32 & 15 \\
\hline 1.53 & 0.34 & 20 \\
\hline 1.62 & 0.36 & 20 \\
\hline 1.4 & 0.35 & 20 \\
\hline 1.76 & 0.32 & 20 \\
\hline 1.92 & 0.35 & 20 \\
\hline 1.6 & 0.32 & 20 \\
\hline 1.87 & 0.375 & 20 \\
\hline 2.27 & 0.35 & 20 \\
\hline 2.24 & 0.32 & 20 \\
\hline 2.45 & 0.35 & 20 \\
\hline 2.52 & 0.36 & 20 \\
\hline 2.62 & 0.375 & 20 \\
\hline 2.66 & 0.38 & 20 \\
\hline & & \\
\hline
\end{tabular}

The values assigned to channel model parameters (transition probabilities), viz. I, II, III, IV... X (defined in Figure 5.3) are listed in Table 5-2. The values for the transition probabilities were chosen with a channel failure rate (CFR) of $5 \%$ in view. The values assumed result in a CFR of $4.94 \%$. We call it Channel Model A. Simulations can be carried out with a range of values assigned to the channel model parameters, depending on the CFR to be considered. Simulations were run for two different scenarios, viz. a) Values of CFR in the vicinity of $1 \%$ and b) Values of CFR in the vicinity of $5 \%$.

Channel Model A (CFR $=4.94 \%)$ has been used for simulations initially, with transition 
probabilities defined in Table 5-2. The probability of the channel being in a bad condition due to Shadowing, Fading or Interference is $4.94 \%$. We have assumed that the probability of occurrence of Shadowing and Fading is the same and hence we have assigned the same value $(0.0125)$ to the parameters $\Pi\left(P_{G S 1}\right)$ and $V\left(P_{G F 1}\right)$ of the channel model.

Later we assume that the probability of occurrence of shadowing is roughly twice that of fading and hence assign values 0.025 and 0.0125 to parameters $I I$ and $V$ respectively. This model has been called Channel Model A1 (CFR = 5.37\%) and is represented in Table 5-3. The probability of moving back to the 'good' state of the channel increases monotonically as we move to a further off state in the channel model. Parameters VII, $\mathrm{XX}$ and $\mathrm{X}\left(\mathrm{P}_{\mathrm{SIG}}\right.$, $\mathrm{P}_{\mathrm{S} 2 \mathrm{G}}$ and $\mathrm{P}_{\mathrm{F1G}}$ ) have been assigned unique values in all the channel models, viz. Channel Model $\mathrm{A}, \mathrm{A} 1$ and $\mathrm{B}$. In all the models $\mathrm{P}_{\mathrm{S} 2 \mathrm{G}}$ has been assigned a higher value than $\mathrm{P}_{\mathrm{S} 1 \mathrm{G}}$.

The CFRs for all the models were obtained by solving the matrix equation $\pi \cdot P_{X Y}=\pi$, where $\pi$ is a row vector (dimension $1 \times 7$ ) of steady-state probability (the steady state probability of being in a particular state out of the seven possible states, viz. G, S1, S2, S3,

Table 5-2: Channel Parameter Values (Channel Model A)

\begin{tabular}{|c|c|c|}
\hline $\begin{array}{c}\text { Transition } \\
\text { Probability }\end{array}$ & Parameter & Value \\
\hline \hline P $_{\text {GG }}$ & I & 0.95 \\
\hline P $_{\text {GS1 }}$ & II & 0.0125 \\
\hline P $_{\text {SIS2 }}$ & III & 0.075 \\
\hline$P_{\text {S2S3 }}$ & IV & 0.05 \\
\hline$P_{\text {GF1 }}$ & V & 0.0125 \\
\hline$P_{\text {FIF }}$ & VI & 0.075 \\
\hline$P_{\text {GI }}$ & VII & 0.025 \\
\hline$P_{\text {SIG }}$ & VIII & 0.925 \\
\hline
\end{tabular}


Table 5-2: Channel Parameter Values (Channel Model A)

\begin{tabular}{|c|c|c|}
\hline $\begin{array}{c}\text { Transition } \\
\text { Probability }\end{array}$ & Parameter & Value \\
\hline \hline P 2 G & $\mathrm{IX}$ & 0.95 \\
\hline P $_{\text {F1G }}$ & $\mathrm{X}$ & 0.925 \\
\hline
\end{tabular}

$F 1, F 2, I$ ) values and $P_{X Y}$ is a transition probability matrix (dimension $7 \times 7$ ) containing probability values of all possible transitions between the given states.

Table 5-3: Channel Parameter Values (Channel Model A1)

\begin{tabular}{|c|c|c|}
\hline $\begin{array}{c}\text { Transition } \\
\text { Probability }\end{array}$ & Parameter & Value \\
\hline \hline$P_{\text {GG }}$ & I & 0.95 \\
\hline$P_{\text {GS1 }}$ & II & 0.025 \\
\hline$P_{\text {SIS2 }}$ & III & 0.2 \\
\hline$P_{\text {S2S }}$ & IV & 0.1 \\
\hline$P_{\text {GFI }}$ & V & 0.0125 \\
\hline$P_{\text {FIF }}$ & VI & 0.1 \\
\hline$P_{\text {GI }}$ & VII & 0.0125 \\
\hline$P_{\text {S1G }}$ & VII & 0.8 \\
\hline$P_{\text {S2G }}$ & IX & 0.9 \\
\hline$P_{\text {FIG }}$ & $\mathrm{X}$ & 0.9 \\
\hline
\end{tabular}

\subsubsection{Effect of variation of the minimum guaranteed bandwidth.}

To study the effect of varying $T_{\min }$ on the steady state performance, the CFR and the MTD were kept fixed and the minimum number of time slots (minimum guaranteed bandwidth), $T_{\min }$, guaranteed to the given user (source) per multiple access frame was varied. The first 
set of simulations was carried out keeping MTD fixed at $33 \mathrm{msec}$ and CFR fixed at $4.94 \%$ (Channel Model A). Another set of simulations was executed setting the value of MTD at 40 msec (less 'sensitive to delay' source as compared to the former) and using Channel Model A $($ CFR = 4.94\%). Finally, simulations were run with MTD $=40 \mathrm{msec}$ and employing Channel Model Al (CFR = 5.37\%). In Channel Model Al the probability of occurrence of shadowing is higher than that of fading and interference, and the probabilities of returning back to the good state from shadowing and fading have been lowered. This results in a slightly higher value of CFR for Channel ModeI A1.

Figure 5-5, Figure 5-6 and Figure 5-7 depict the performance curves obtained for three different values of $T_{\min }$, viz. $T_{\min }=20,25$ and 27 . MTD and CFR were kept fixed at 33 msec and $4.94 \%$ respectively. For each value of $T_{\min }$, utilization $(U)$ was varied and the corresponding CLP, MBO and MWT values were recorded. As is clear from Figure 5-5, 56 and 5-7, Cell Loss Probability (CLP), Mean Buffer Occupancy (MBO) and Mean Waiting Time (MWT) are found to be lower for higher values of $T_{\min }$. This is because $T_{\min }$ affects the residence time of the cells in the terminal buffer. Greater the value of $T_{\text {min }}$, greater is the possibility of more cells being released from the buffer per multiple access frame, the reason being that under conditions of high bandwidth demand by the other sources, the given source is assured of a greater number of slots in the multiple access frame. The variations in the MBO and the MWT with utilization are found to be minimal due to the time sensitive nature of the source. With a decrease in the minimum guaranteed bandwidth, $T_{\min }$, the MBO would tend to increase due to the cells getting stacked in the buffer. However, the increase would only be minimal because the stacked cells get discarded as soon as their residence time in the buffer exceeds the MTD. Following the same reasoning, the increase in the MWT with utilization is minimal for time sensitive sources. The change in the MBO and MWT would tend to become significant with an increase in the value of the MTD and a decrease in the value of the $T_{\min }$. The variations in 


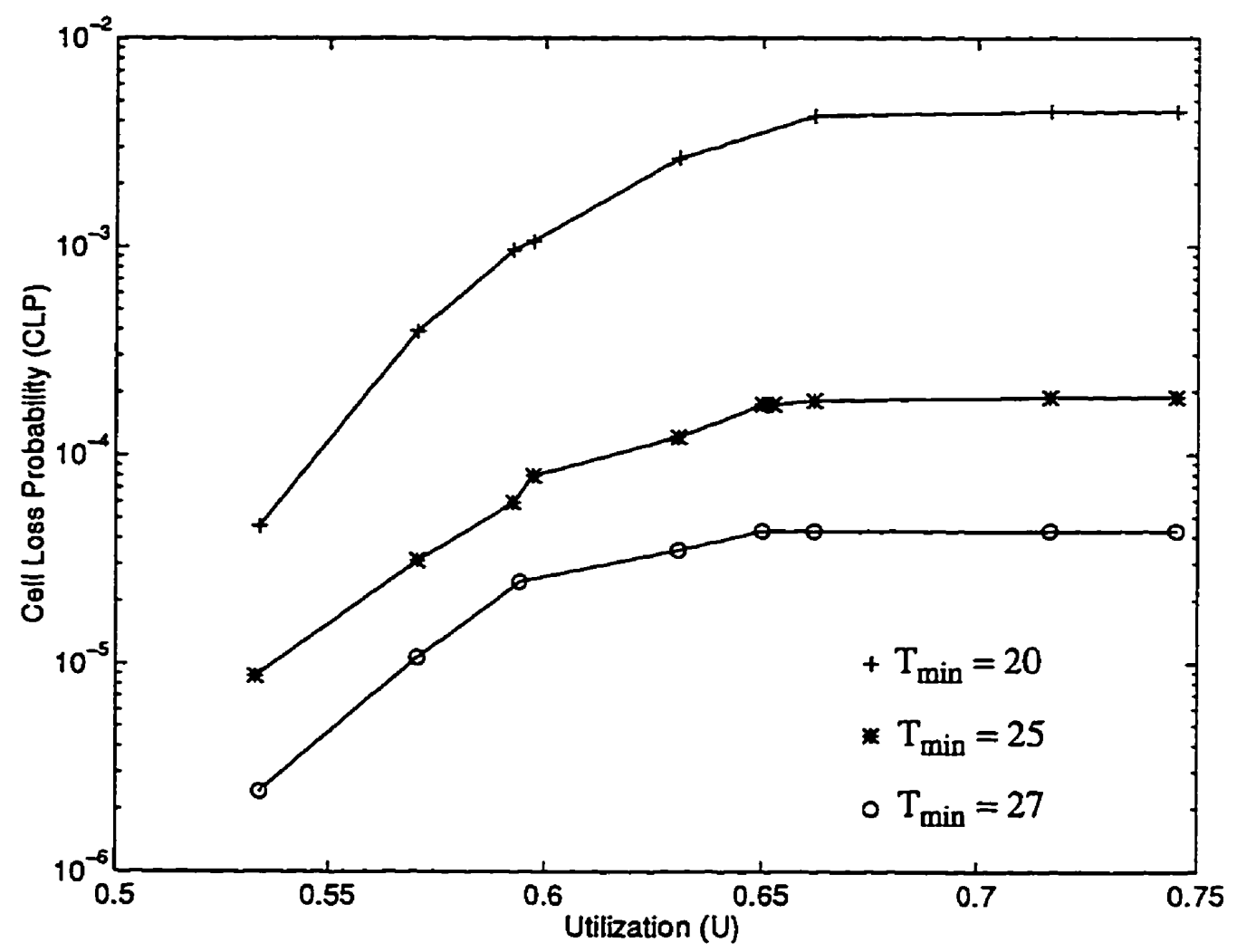

Figure 5-5: Cell Loss Probability versus Utilization for a VBR Video Source modelled with 20 On/Off Mini-Sources $\left(N_{s}\right), A R=0.375$, Mean ON duration $=0.033 \mathrm{sec}$ and $M B R=$ 2.06 Mbps. MTD $=33 \mathrm{msec}$, CFR $=4.94 \%$. 


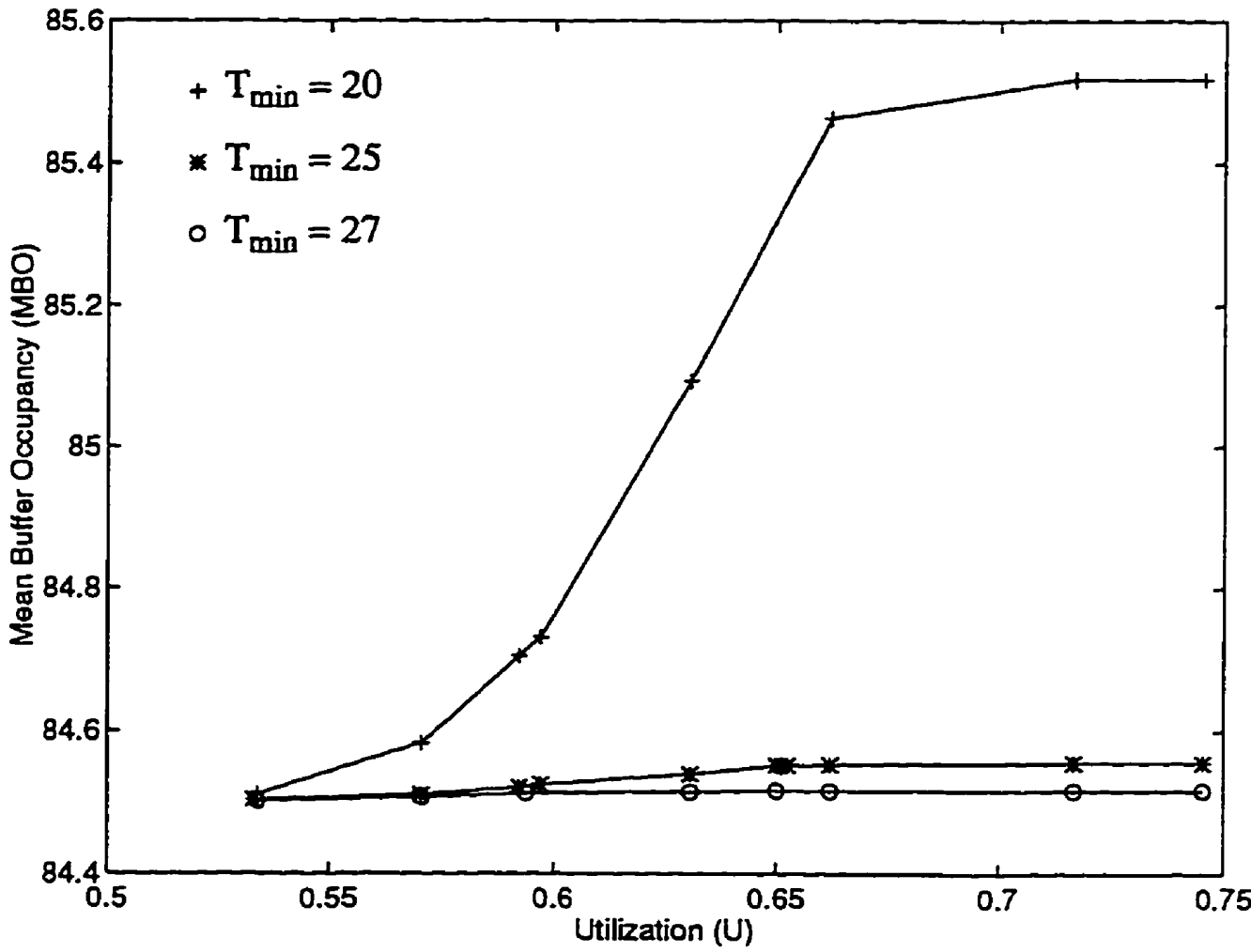

Figure 5-6: Mean Buffer Occupancy versus Utilization for a VBR Video Source modelled with $20 \mathrm{On} /$ Off Mini-Sources $\left(\mathrm{N}_{s}\right), A R=0.375$, Mean $\mathrm{ON}$ duration $=0.033 \mathrm{sec}$ and MBR $=2.06$ Mbps. $\mathrm{MTD}=33 \mathrm{msec}, \mathrm{CFR}=4.94 \%$. 


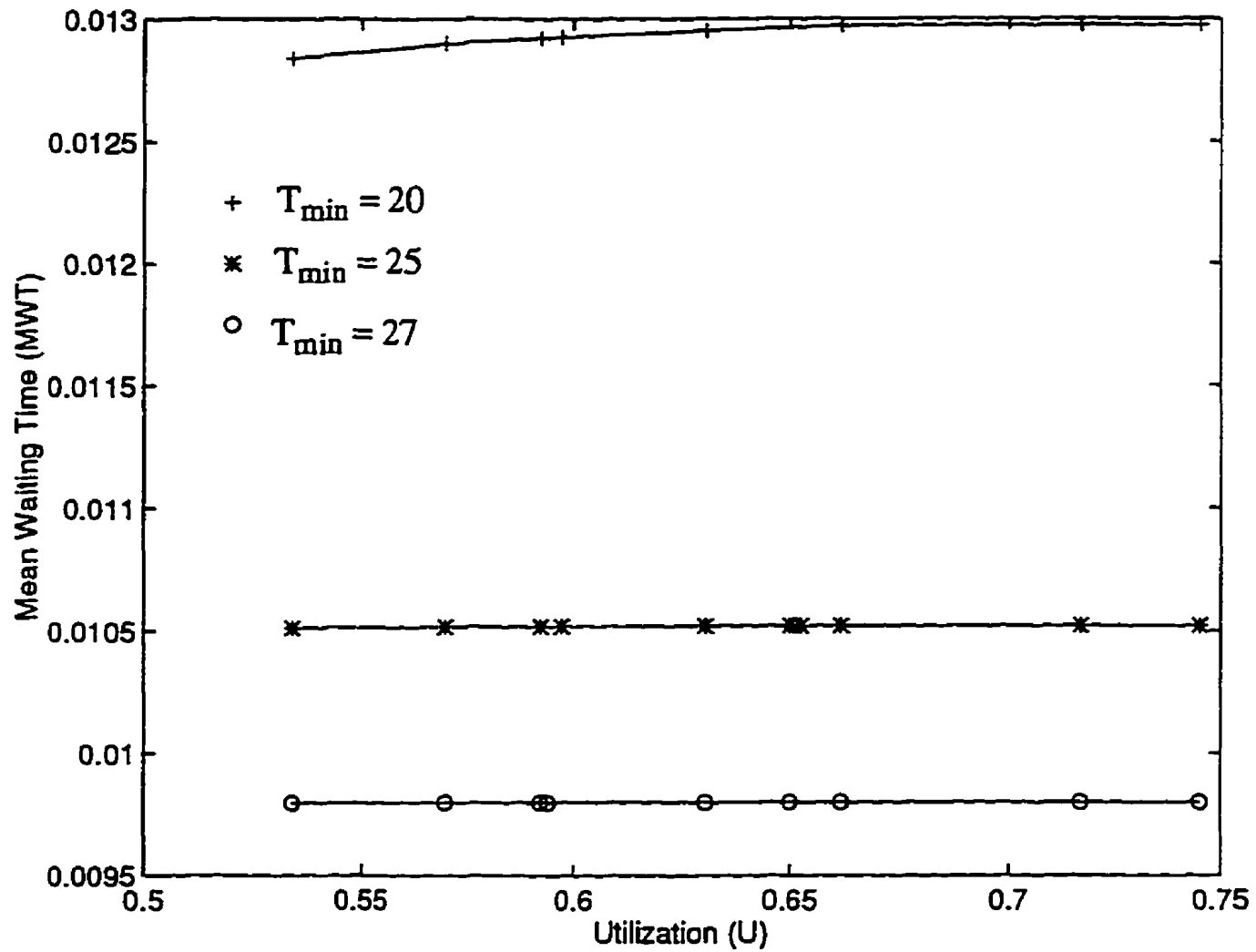

Figure 5-7: Mean Waiting Time versus Utilization for a VBR Video Source modelled with $20 \mathrm{On} /$ Off Mini-Sources $\left(\mathrm{N}_{s}\right), A R=0.375$, Mean $\mathrm{ON}$ duration $=0.033 \mathrm{sec}$ and MBR $=2.06 \mathrm{Mbps}$. MTD $=33 \mathrm{msec}$, CFR $=4.94 \%$. 
the CLP, MBO and the MWT with utilization have been plotted. The utilization was varied by unique combinations of the previously mentioned video sources.

In the second set, MTD was changed to $40 \mathrm{msec}$, keeping other parameters fixed. Steady state performance curves were then obtained for three different values of $T_{\min }$, viz. 18,20 and 22. It can be observed that a $T_{\min }=18$, for a source with $M T D=40 \mathrm{msec}$ gives a better steady state performance (lower CLP) than a source with MTD = $33 \mathrm{msec}$ that has been assigned a $T_{\min }=20$. Moreover, it is observed that assignment of a proper $T_{\min }$ to a source with a lower MTD (more time sensitive) is more critical than that to a source with a high MTD (less time sensitive).Fig 5-8 plots the variation of CLP with utilization for a source with MTD $=40 \mathrm{msec}$ and for a CFR of $4.94 \%$. Fig 5-9 plots the variation of MBO with utilization. The increase in the $\mathrm{MBO}$ with utilization for $\mathrm{T}_{\min }=18$ is quite significant as compared to the cases when $T_{\min }=20$ and $T_{\min }=22$.

Finally, simulations were executed employing Channel Model A1 (CFR $=5.37 \%$ ), keeping MTD constant ( $40 \mathrm{msec}$ ). Fig. 5-10 plots the variation of CLP with utilization for a source with MTD $=40 \mathrm{msec}$ and for a CFR of $5.37 \%$. Curves have been plotted for two different values of $T_{\min }$, viz. 18 and 23. In Channel Model A1, the probability of occurrence of shadowing is twice that of fading. Also, the probability of occurrence of interference has been lowered as compared to that in Channel Model A. The increase in the probability of occurrence of shadowing in turn implies that the channel would tend to take more number of cycles to recover to a 'good' condition from a 'bad' condition as compared to the case when fading and shadowing occurred with equal probability. This causes a slight increase in the CFR. Comparing the CLP vs. U. curves for a source with MTD $=40 \mathrm{msec}$, that has been assigned a $T_{\min }=18$, for a) Channel Model A1 (CFR of $5.37 \%$ ), and b) Channel Model A (CFR of 4.94\%), we observe that the latter gives a slightly better performance (lower CLP) than the former. This difference is because of the 


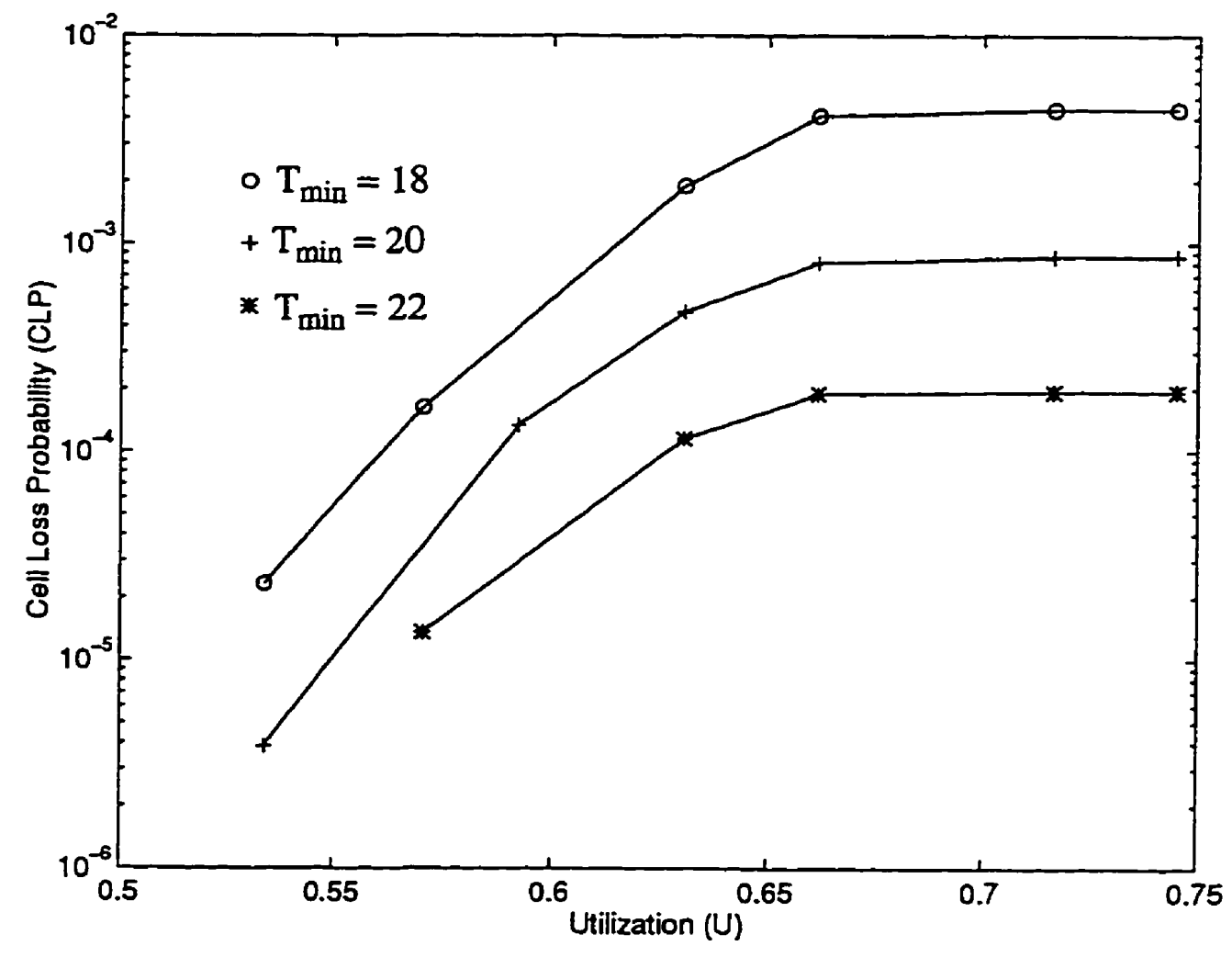

Figure 5-8: Cell Loss Probability versus Utilization for a VBR Video Source modelled with $20 \mathrm{On} / \mathrm{Off}$ Mini-Sources $\left(\mathrm{N}_{\mathrm{s}}\right), \mathrm{AR}=0.375$, Mean $\mathrm{ON}$ duration $=0.033$ sec and MBR $=2.06$ Mbps. MTD $=40$ msec, CFR $=4.94 \%$. 


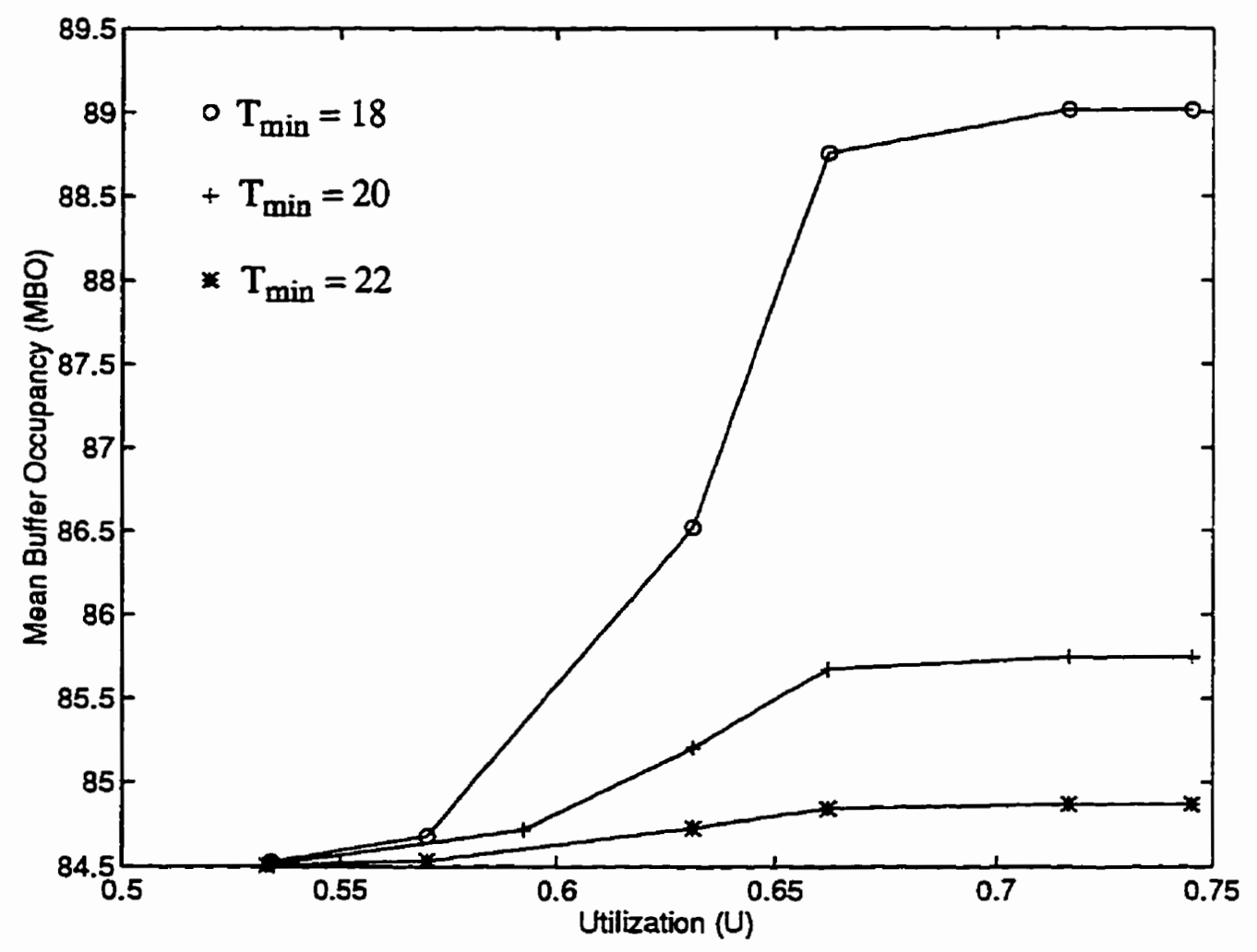

Figure 5-9: Mean Buffer Occupancy versus Utilization for a VBR Video Source modelled with 20 On/Off Mini-Sources $\left(N_{s}\right), A R=0.375$, Mean ON duration $=0.033 \mathrm{sec}$ and MBR $=2.06 \mathrm{Mbps}$. MTD $=40 \mathrm{msec}$, CFR $=4.94 \%$. 


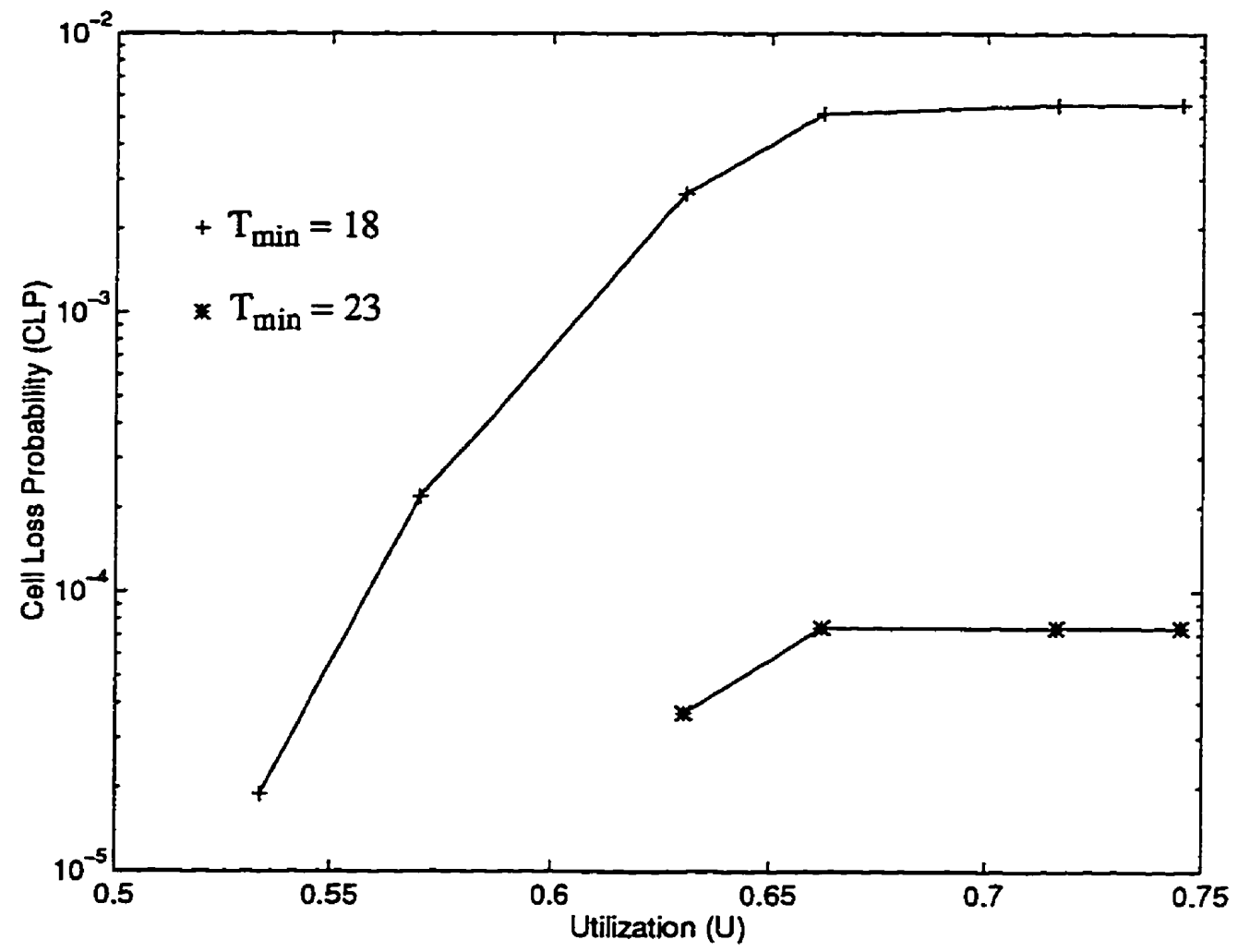

Figure 5-10: Cell Loss Probability versus Utilization for a VBR Video Source modelled with $20 \mathrm{On} /$ Off Mini-Sources $\left(N_{s}\right), A R=0.375$, Mean $O N$ duration $=0.033 \mathrm{sec}$ and MBR $=2.06$ Mbps. MTD $=40$ msec, CFR $=5.37 \%$. 
fact that shadowing is the main cause of channel failure in Channel Model A1, owing to which the channel is blocked for more number of cycles consecutively, as compared to Channel Model A.

It is observed from Figure 5-5 and 5-10 that a source with a higher value of the MTD (40 msec) exhibits a better performance (lower CLP) at $T_{\min }=23$ than a source with a lower MTD (33 msec) at $T_{\min }=25$, although the CFR in case of the former $(C F R=5.37 \%$ ) is higher than the latter (CFR = 4.94\%).

\subsubsection{Effect of variation of the Maximum Transfer Delay.}

With the same video sources, a fixed value of $T_{\min }=20$ and using Channel Model $A$, simulations were carried out to obtain performance curves for two different values of the MTD, viz. $33 \mathrm{msec}$ and $40 \mathrm{msec}$, plotted in Figure 5-11, Figure 5-12 and Figure 5-13. Figure 5-11 shows that the CLP decreases with an increase in the MTD. As stated earlier, time-sensitive cells are discarded if not delivered within their MTD. A high value of the MTD implies lower time-sensitivity. This increases the likelihood of the ATM cells sustaining a longer residence in the terminal buffer. This in turn implies that there is a greater probability (as compared to the RTT with lower MTD) of a given cell (from the time it is stacked in the terminal buffer) being transmitted from the buffer in one of the subsequently available multiple access frames, before being discarded, on account of being delayed longer than their MTD.

The MBO increases with an increase in the MTD as shown in Figure 5-12. This is because an increased MTD results in an increase in the permissible duration of the residency of an ATM cell in the terminal buffer. Similarly, the MWT of an ATM cell increases with the MTD, which is clear from Figure 5-13. 


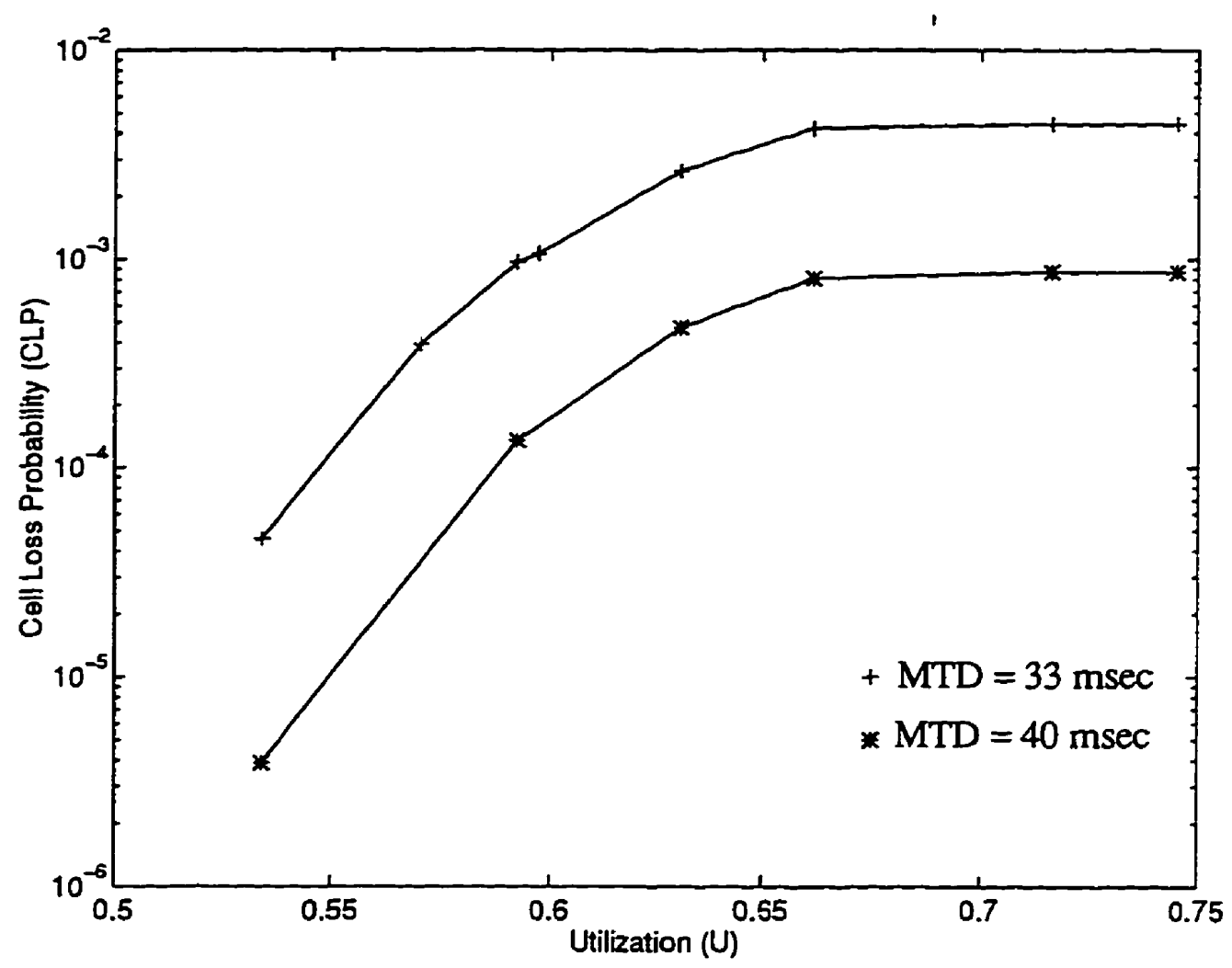

Figure 5-11: Cell Loss Probability versus Utilization for a VBR Video Source modelled with $20 \mathrm{On} / \mathrm{Or}$ Mini-Sources $\left(\mathrm{N}_{\mathrm{s}}\right), \mathrm{AR}=\mathbf{0 . 3 7 5}$, Mean $\mathrm{ON}$ duration $=0.033 \mathrm{sec}$ and MBR $=2.06$ Mbps. $T_{\min }=20, C F R=4.94 \%$. 


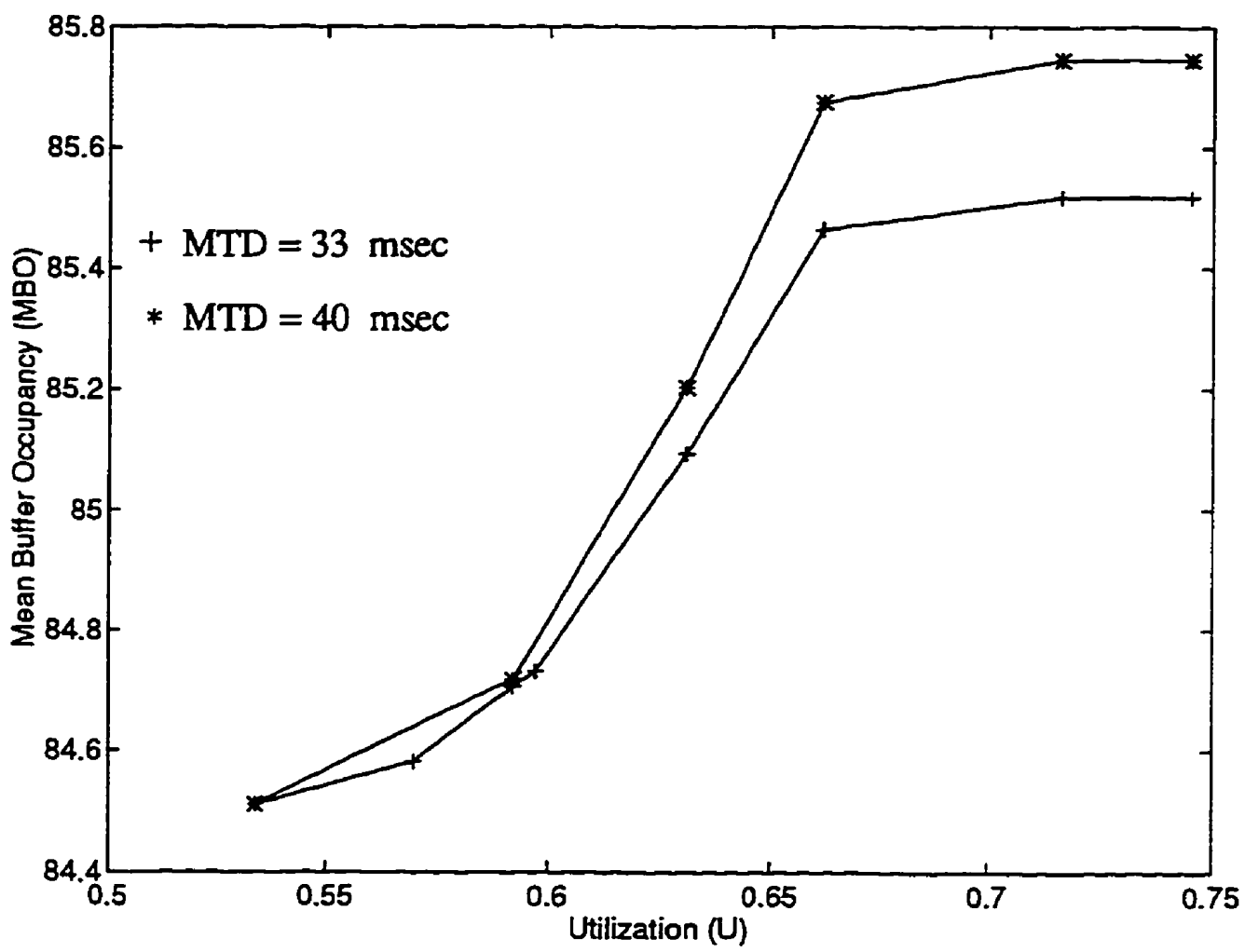

Figure 5-12: Mean Buffer Occupancy versus Utilization for a VBR Video Source modelled with $20 \mathrm{On} / \mathrm{Of}$ Mini-Sources $\left(\mathrm{N}_{s}\right), A R=0.375$, Mean $\mathrm{ON}$ duration $=0.033 \mathrm{sec}$ and MBR $=2.06$ Mbps. $T_{\min }=20$, CFR $=4.94 \%$. 


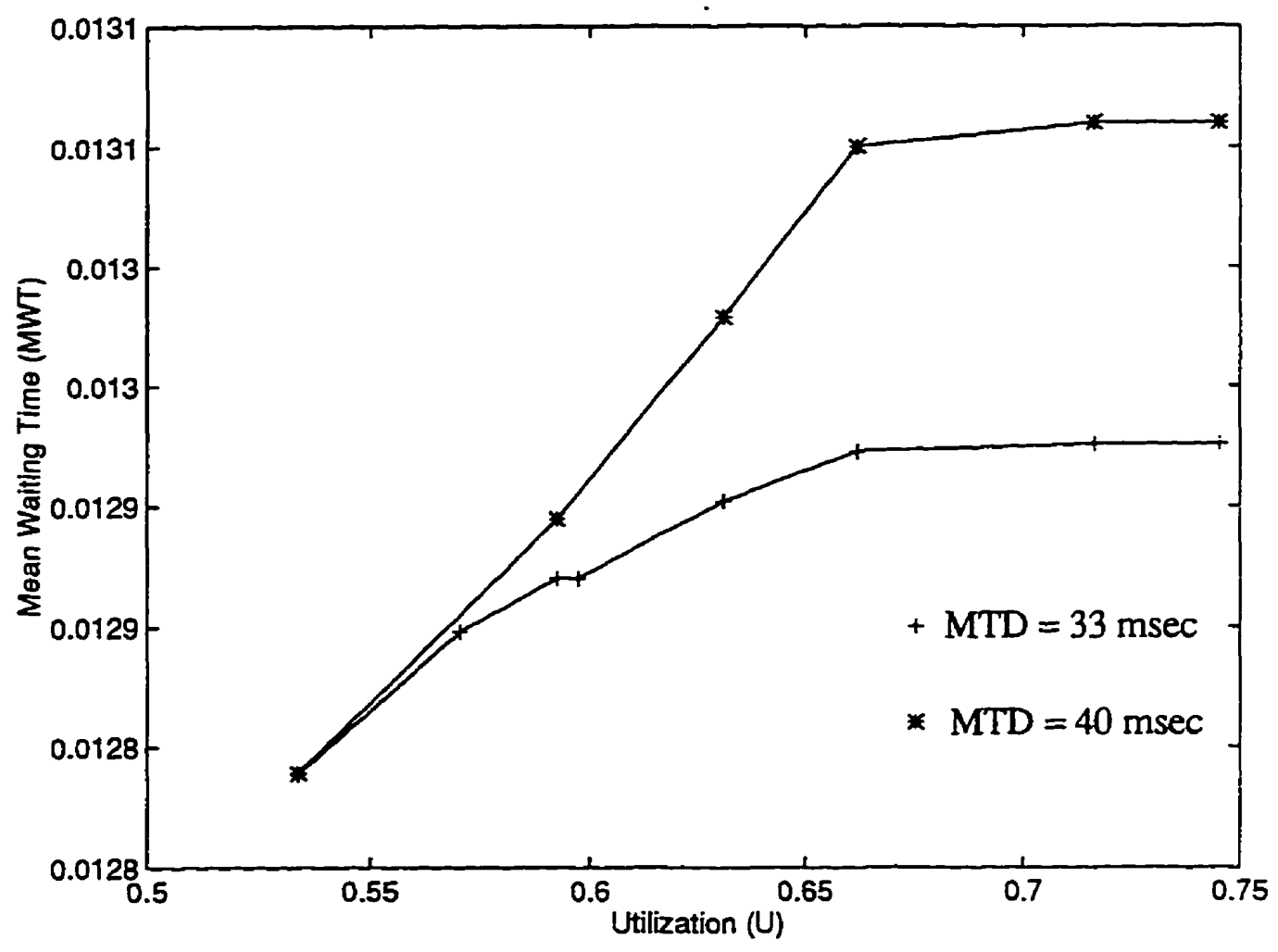

Figure 5-13: Mean Waiting Time versus Utilization for a VBR Video Source modelled with $20 \mathrm{On} /$ Off Mini-Sources $\left(\mathrm{N}_{\mathrm{s}}\right), A R=0.375$, Mean $\mathrm{ON}$ duration $=0.033 \mathrm{sec}$ and $M B R=2.06 \mathrm{Mbps} . \mathrm{T}_{\min }=20, \mathrm{CFR}=4.94 \%$. 


\subsection{Simulations with VBR MPEG Compressed Video Data exhibiting self-similar characteristics.}

In this section, we present the results of another set of simulations carried out with traffic sources modelled using the data generated from self-similar traffic characteristics model, discussed earlier in section 4.4. The data was available in the form of bytes per I, B and P frames, each of $33 \mathrm{msec}$ duration. All of the modelled video sources generated VBR MPEG Compressed Video data with the following characteristics:

Let the burstiness, ' $b$ ', of a given source be defined as the ratio of the maximum bit-rate to the mean bit-rate of the source.

The duration of each video frame was $33 \mathrm{msec}$.

The given video source (the 'given user') has an average bit-rate of $1.145 \mathrm{Mbps}$ and burstiness, $b=8.83$.

The remaining sources ('other sources') were modelled as shown in Table 5-4.

Table 5-4: Video Sources Based on VBR Compressed Video Data

\begin{tabular}{|c|c|}
\hline MBR (Mbps) & b \\
\hline \hline 2.45 & 3.97 \\
\hline 1.8547 & 4.41 \\
\hline 1.3685 & 2.68 \\
\hline 1.1374 & 2.35 \\
\hline 4.5173 & 4.52 \\
\hline 3.2576 & 5.26 \\
\hline 4.11 & 3.73 \\
\hline 2.056 & 7.47 \\
\hline 2.06 & 8.22 \\
\hline
\end{tabular}


Table 5-4: Video Sources Based on VBR Compressed Video Data

\begin{tabular}{|c|c|}
\hline MBR (Mbps) & b \\
\hline \hline 3.928 & 4.38 \\
\hline
\end{tabular}

\subsubsection{Effect of variation of the Maximum Transfer Delay.}

The minimum guaranteed bandwidth was kept fixed at $T_{\min }=30$. The channel was modelled using Channel Model A. The initial set of simulations were carried out keeping the MTD fixed at 33msec. Later, another set of simulations was executed keeping MTD fixed at 50 msec. A similar phenomenon is found to occur as in the case of the ON/OFF markov mini-sources model. The CLP is found to decrease with an increase in the MTD. Figure 5-14 presents the CLP performance curves under both the above mentioned conditions. The saturation is found to be attained for utilization values above $80 \%$. It is observed that the difference between the CLP saturation values for MTD $=33 \mathrm{msec}$ and MTD $=50 \mathrm{msec}$ is much greater for the case of MPEG-4 sources with self-similar video characteristics as compared to that of ON/OFF markov mini-sources. This is owing to a considerably higher burstiness in the bit-rate of the former.

\subsubsection{Effect of variation of Channel Parameters}

All the simulations carried out till this point adhered to Channel Model A and Al defined in section 5.3. This section presents simulation results with improved channel conditions. The values assigned to channel model parameters, viz. I, II, III, IV... X (defined in Figure 5.3) are listed in Table 5-5. This results in a CFR (channel failure rate) of 1.03\%. Let us

Table 5-5: Channel Parameter Values (Channel Model B)

\begin{tabular}{|c|c|c|}
\hline $\begin{array}{c}\text { Transition } \\
\text { Probability }\end{array}$ & Parameter & Value \\
\hline \hline $\mathrm{P}_{\mathrm{GG}}$ & $\mathrm{I}$ & 0.99 \\
\hline $\mathrm{P}_{\mathrm{GS} 1}$ & $\mathrm{II}$ & 0.0025 \\
\hline
\end{tabular}




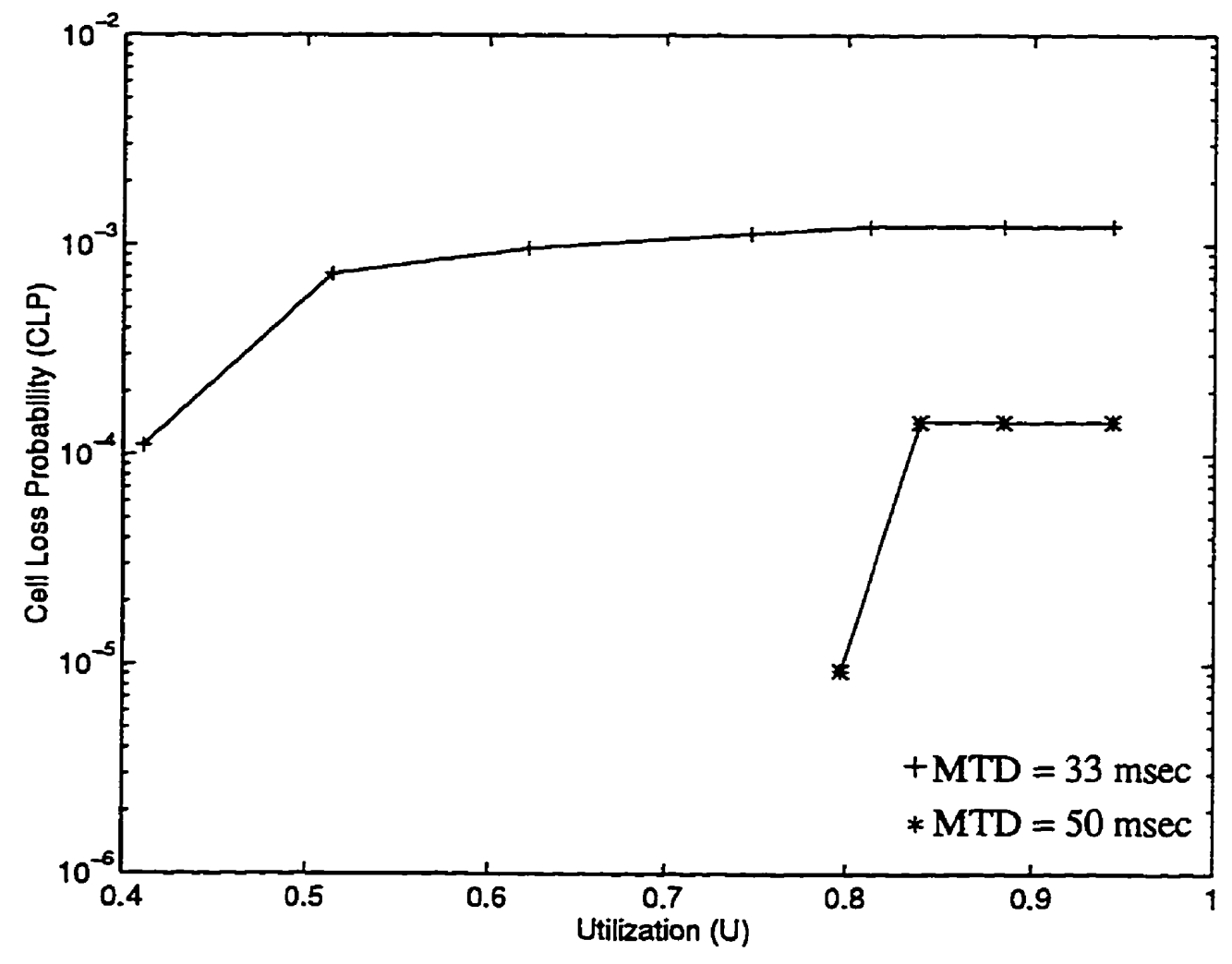

Figure 5-14: Cell Loss Probability versus Utilization for VBR MPEG Compressed Video Source. MBR $=1.145 \mathrm{Mbps}$, burstiness ' $b$ ' $=8.83 . \mathrm{T}_{\min }=30, \mathrm{CFR}=4.94 \%$. 
Table 5-5: Channel Parameter Values (Channel Model B)

\begin{tabular}{|c|c|c|}
\hline $\begin{array}{c}\text { Transition } \\
\text { Probability }\end{array}$ & Parameter & Value \\
\hline \hline$P_{\text {SIS2 }}$ & III & 0.05 \\
\hline$P_{\text {S2S3 }}$ & IV & 0.0125 \\
\hline$P_{\text {GF }}$ & V & 0.005 \\
\hline$P_{\text {FIF }}$ & VI & 0.05 \\
\hline$P_{\text {GI }}$ & VII & 0.0025 \\
\hline$P_{\text {S1G }}$ & VIII & 0.95 \\
\hline$P_{\text {S2G }}$ & IX & 0.975 \\
\hline$P_{\text {F1G }}$ & $\mathrm{X}$ & 0.95 \\
\hline
\end{tabular}

refer to this channel model as Channel Model B.

There is a noticeable improvement in the CLP values with a decrease in the channel failure rate from $4.94 \%$ to $1.03 \%$, as shown in Figure 5-15. It is observed that there is a slight decrease in the CLP value with an increase in the utilization (for utilization values between $70 \%$ and $80 \%$ ), for the case of Channel Model B. Such a phenomenon can occur when the other sources competing for bandwidth with the given user exhibit a significantly greater burstiness in their bit-rates at lower values of utilization than at higher utilization values. Steady-state is found to be attained for utilization above $80 \%$ in both the cases. A similar variation is observed in the MBO with utilization, as shown in Figure 5-16. MWT for the two cases is compared in Figure 5-17. MWT is found to remain more or less constant for utilization values above $50 \%$. With the improvement in the channel conditions, there is a decrease in the number of retransmissions of the ATM cells stacked in the buffer. This directly affects the residence of the cells in the buffer, resulting in a decrease in the average time for which a video cell is held in the buffer. MWT values in the case of Channel Model B are found to be lower than those for the Channel Model A by 


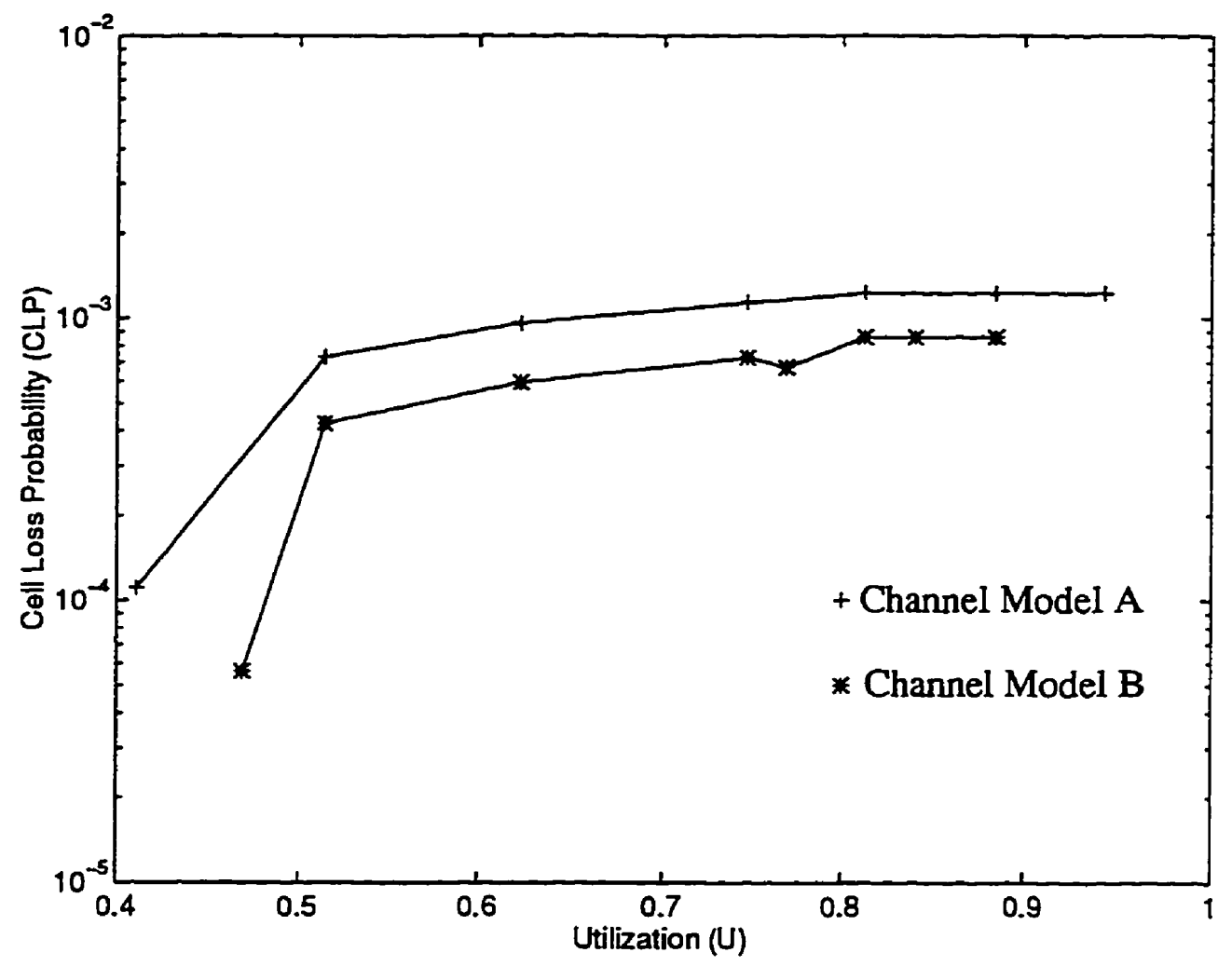

Figure 5-15: Cell Loss Probability versus Utilization for VBR MPEG Compressed Video Source. MBR = 1.145 Mbps, burstiness ' $b$ ' $=8.83 . T_{\min }=30$, MTD $=33 \mathrm{msec}$. 


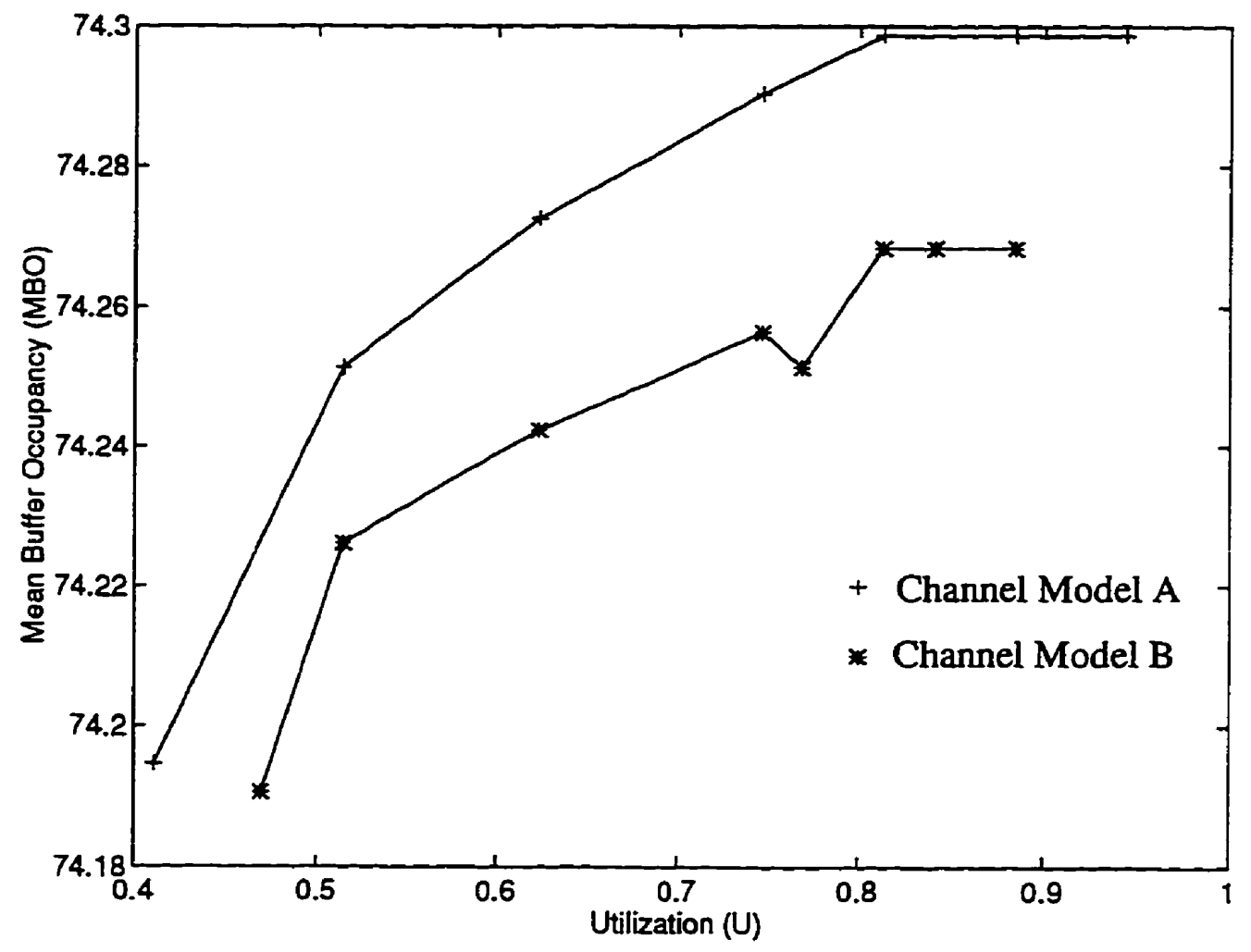

Figure 5-16: Mean Buffer Occupancy versus Utilization for VBR MPEG Compressed Video Source. $M B R=1.145 \mathrm{Mbps}$, burstiness ' $b$ ' $=8.83 . T_{\min }=30, M T D=33 \mathrm{msec}$. 


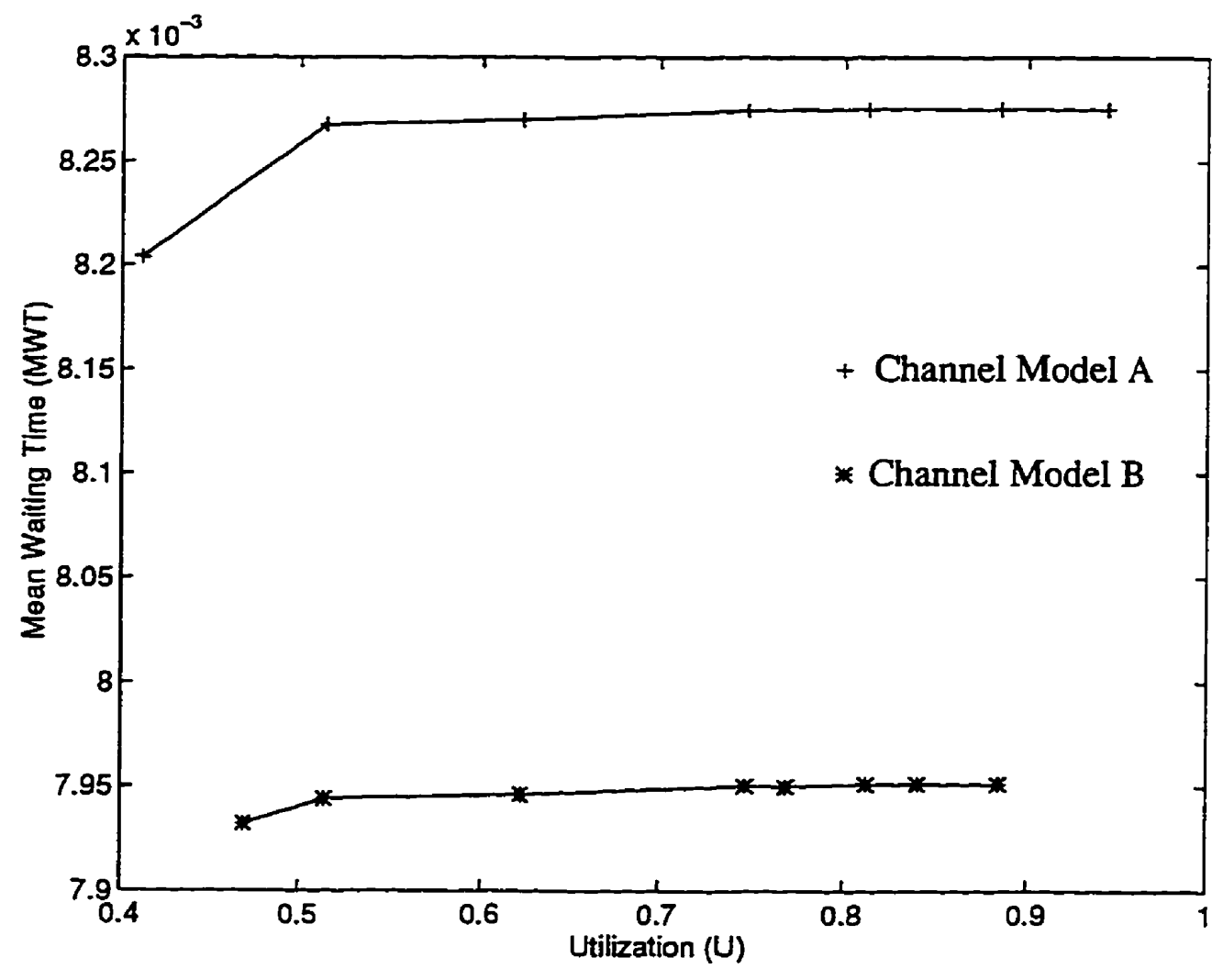

Figure 5-17: Mean Waiting Time versus Utilization for VBR MPEG Compressed Video Source. $M B R=1.145$ Mbps, burstiness ' $b$ ' $=8.83 . \Upsilon_{\min }=30, M T D=33 \mathrm{msec}$. 
around $0.3 \mathrm{msec}$.

\subsubsection{Effect of Network Capacity}

All the simulations carried out so far considered a network capacity of $31.45 \mathrm{Mbps}$. In this section we compare the simulation results for three different scenarios corresponding to network capacities of 15 Mbps, 24 Mbps and 31.45 Mbps. As usual, under all the three scenarios, network utilization values were increased gradually by combinations of the simulated VBR video sources. The above changes in the network capacity were found to impact the performance curves significantly. Figure 5-18 presents the CLP vs. U. curves for the three cases. As expected, the network with 15 Mbps capacity exhibits the poorest performance and in contrast the best performance is available with the $31.45 \mathrm{Mbps}$ capacity network. A ten-fold degradation is observed in the CLP values when the network capacity is decreased from 31.45 Mbps to $24 \mathrm{Mbps}$ and further when the network capacity is decreased from $24 \mathrm{Mbps}$ to $15 \mathrm{Mbps}$. In the cases of $15 \mathrm{Mbps}$ and $24 \mathrm{Mbps}$ network capacity, we come across very high CLP values at sufficiently low values of utilization (in the range of $30-40 \%$ ). This results in a poor performance for VBR video traffic, for which the desired CLP values fall in the range of $10^{-4}$ to $10^{-6}$. For a network capacity of 24 Mbps, a steep rise in the CLP value is observed (for utilization values in the vicinity of 30), with a very small increase in the network utilization. This is because of a vast difference in the level of burstiness of the video sources corresponding to the two values (more or less equal in magnitude) of the network utilization.

Figure 5-19 compares the variations in the MBOs for the three network capacities. There is a much wider variation in the $\mathrm{MBO}$ of the given user under the condition of $15 \mathrm{Mbps}$ network capacity. The MBO is found to increase in larger steps as the network utilization is increased. Also, the saturation is attained at very high values of $U$ (above 90\%). In the case of $24 \mathrm{Mbps}$ network capacity, the variation in the MBO with $U$ is small compared to 


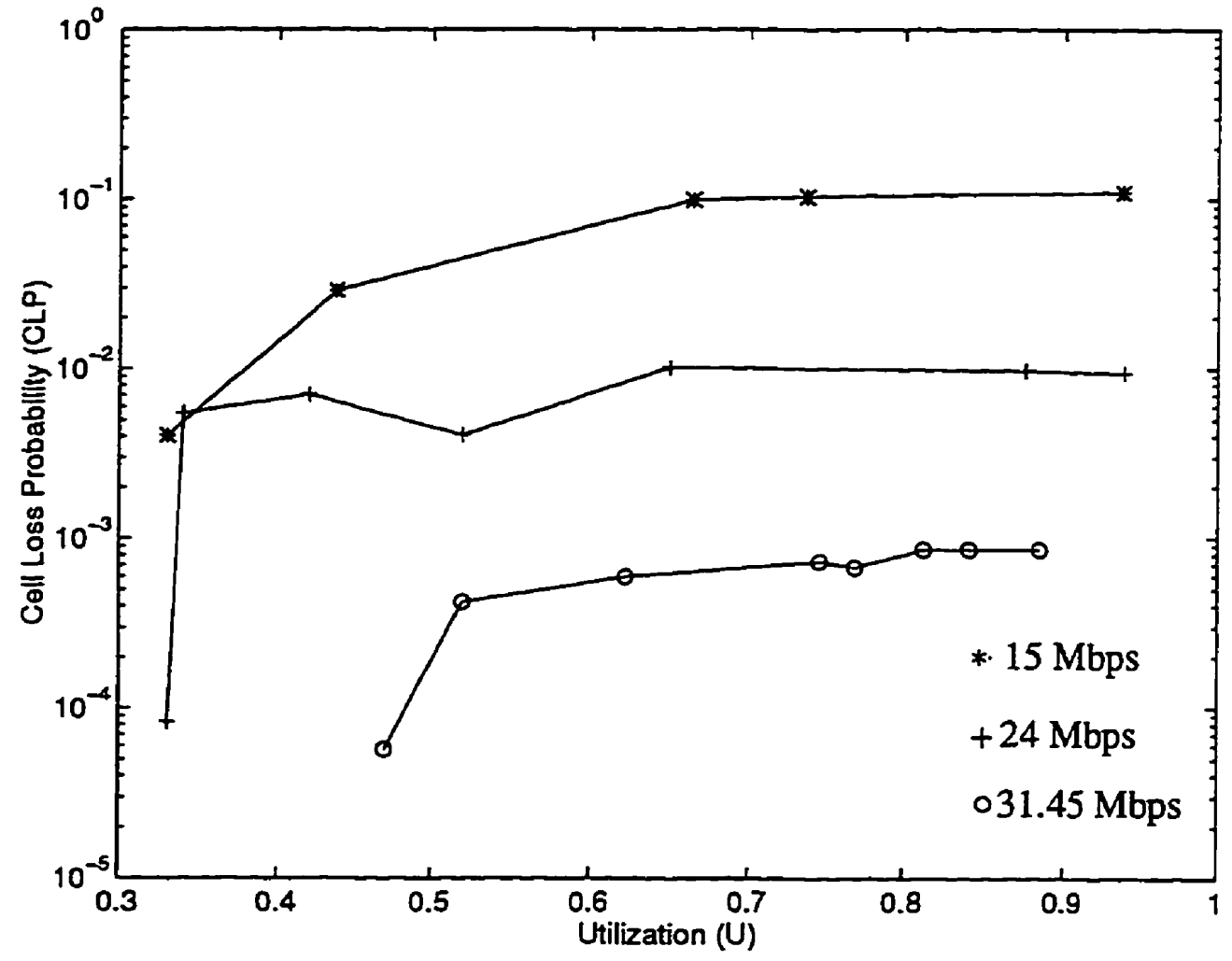

Figure 5-18: Cell Loss Probability versus Utilization for VBR MPEG Compressed Video

Source. $\mathrm{MBR}=1.145 \mathrm{Mbps}$, burstiness ' $b$ ' $=8.83 . \mathrm{T}_{\min }=30, \mathrm{CFR}=1.03 \%$, MTD $=33$ msec. 


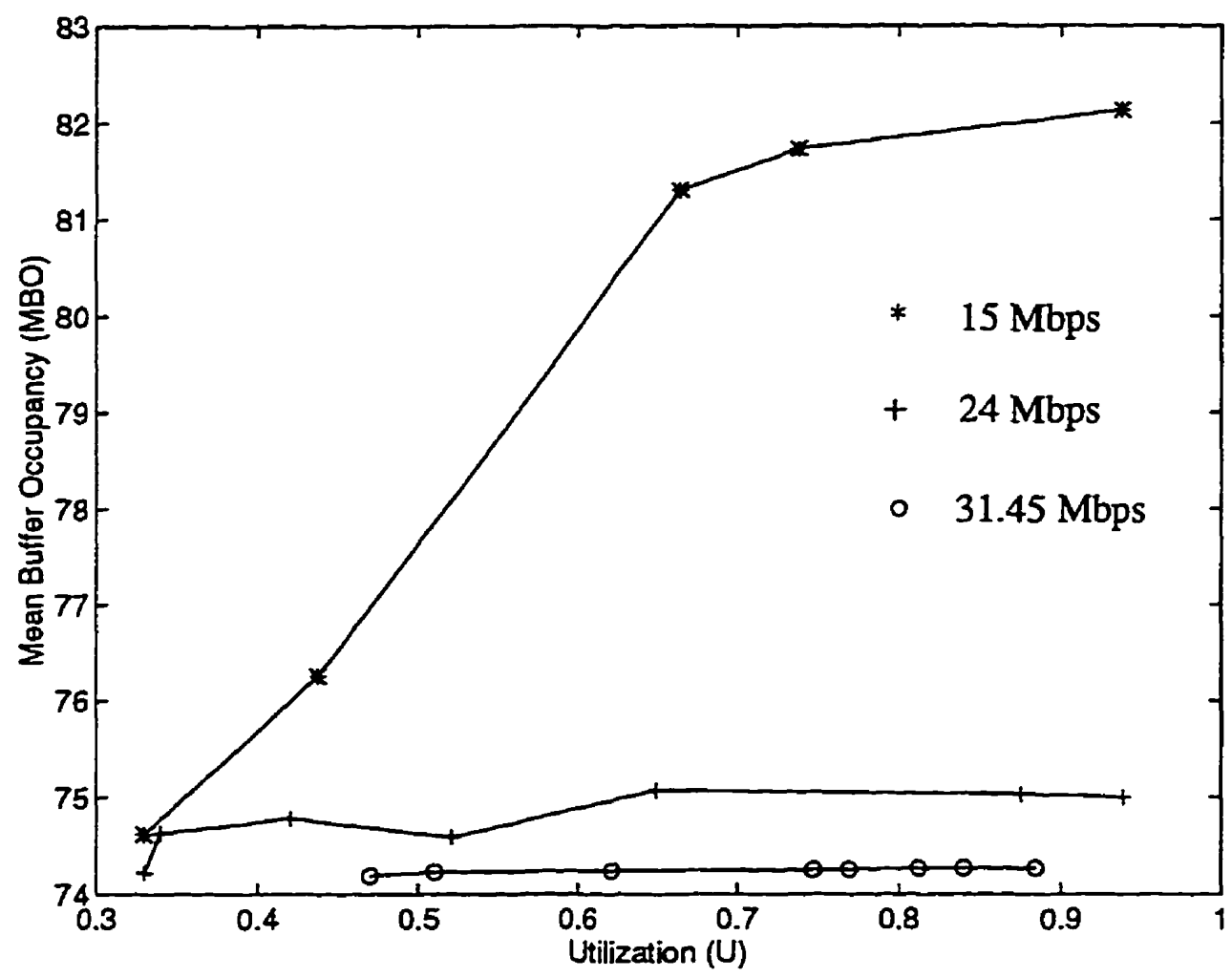

Figure 5-19: Mean Buffer Occupancy versus Utilization for VBR MPEG Compressed Video Source. $M B R=1.145 \mathrm{Mbps}$, burstiness ' $b$ ' $=8.83 . T_{\min }=30, C F R=1.03 \%$, MTD $=33 \mathrm{msec}$. 
the previous case. Saturation is found to be achieved at lower values of $U$ (above 65\%) as compared to the condition of $15 \mathrm{Mbps}$ network capacity. For the network with $31.45 \mathrm{Mbps}$ capacity, the MBO varies the least with utilization and appears more or less constant when compared to the former.

Similar observations as above were made in the MWT vs. U curves, shown in Figure 5-20. The variation in the MWT with $U$ was found to reduce remarkably with an increase in the network capacity (from $15 \mathrm{Mbps}$ to $31.45 \mathrm{Mbps}$ ). The MWT for $15 \mathrm{Mbps}$ network was found to vary over a wide range (roughly between $12 \mathrm{msec}$ and $15 \mathrm{msec}$ ) with the network utilization.

\subsubsection{Effect of the nature of the given video source}

We used two separate video sources of varying natures to simulate the given user traffic. Figure 5-21, Figure 5-22 and Figure 5-23 compare the steady state performances, as seen by the two sources. It is interesting to note that an MPEG compressed video source with 1.145 Mbps MBR, burstiness (b) $=8.83$ exhibits a much higher CLP (more than tenfold higher) than the source (derived from an ON/OFF markov mini-sources model) with 2.06 Mbps MBR, with burstiness (b) $=2.67$, despite the fact that the former has been assigned a higher $\mathrm{T}_{\min }(30)$ than the latter (27), all other factors remaining identical. This highlights the important role played by the burstiness of a VBR video source in determining the steady state performance. The MBO and the MWT observed in case of the latter is however higher due to a higher Mean Bit Rate (MBR). 


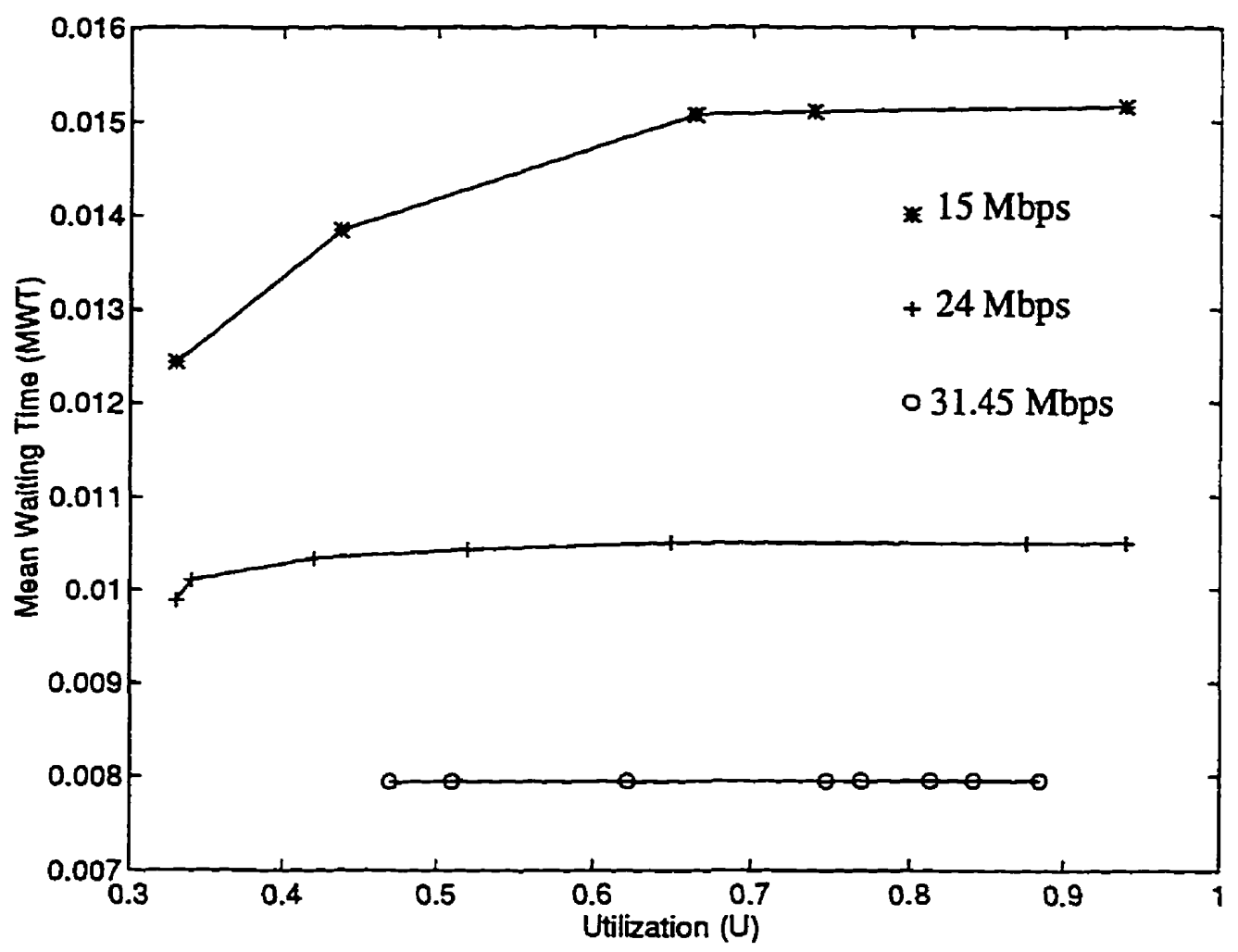

Figure 5-20: Mean Waiting Time versus Utilization for VBR MPEG Compressed Video Source. $M B R=1.145 \mathrm{Mbps}$, burstiness ' $b$ ' $=8.83 . \mathrm{T}_{\min }=30, \mathrm{CFR}=1.03 \%$, MTD = 33 msec. 


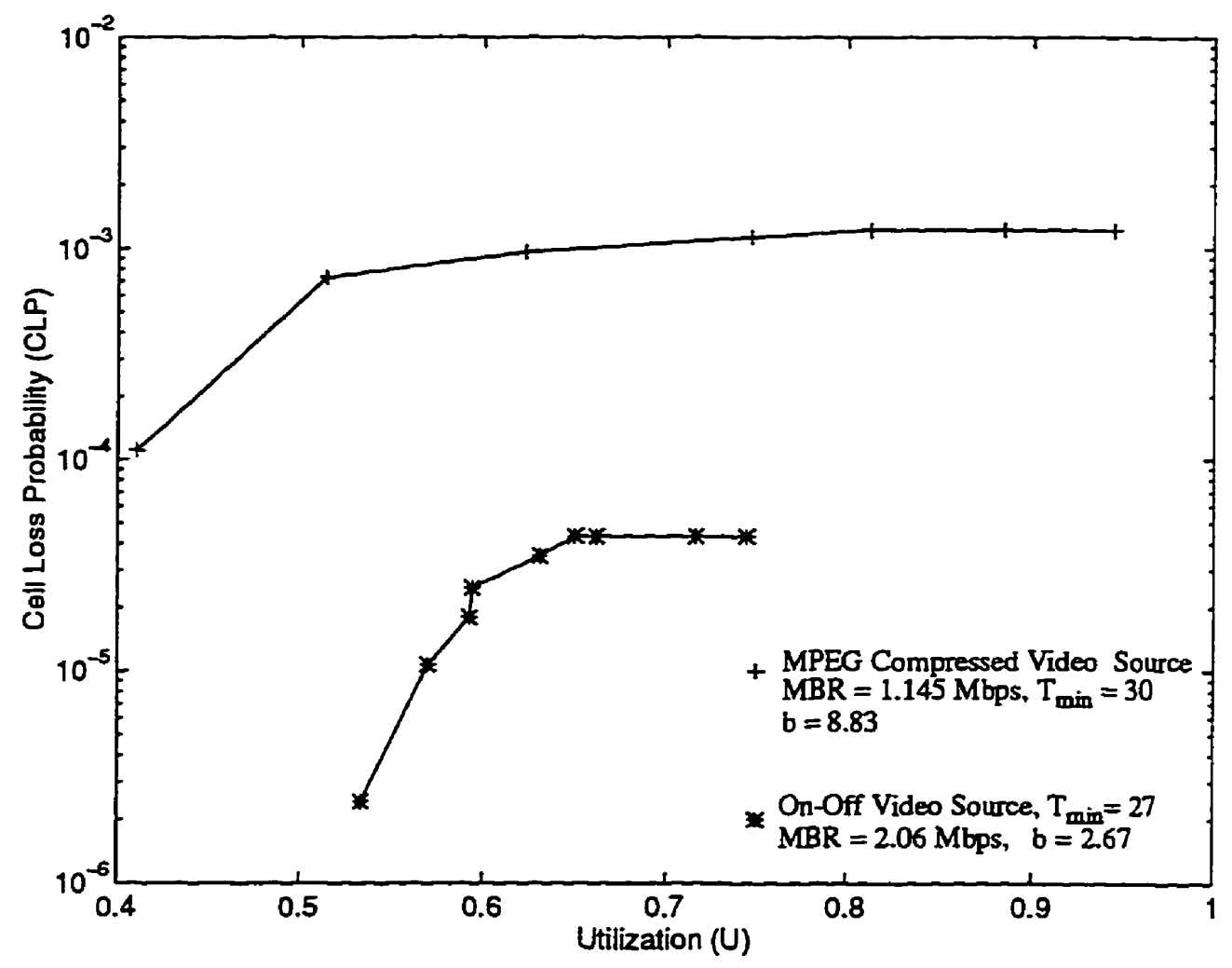

Figure 5-21: Cell Loss Probability versus Utilization for VBR MPEG Compressed Video Source. $C F R=4.94 \%$, MTD $=33 \mathrm{msec}$. 


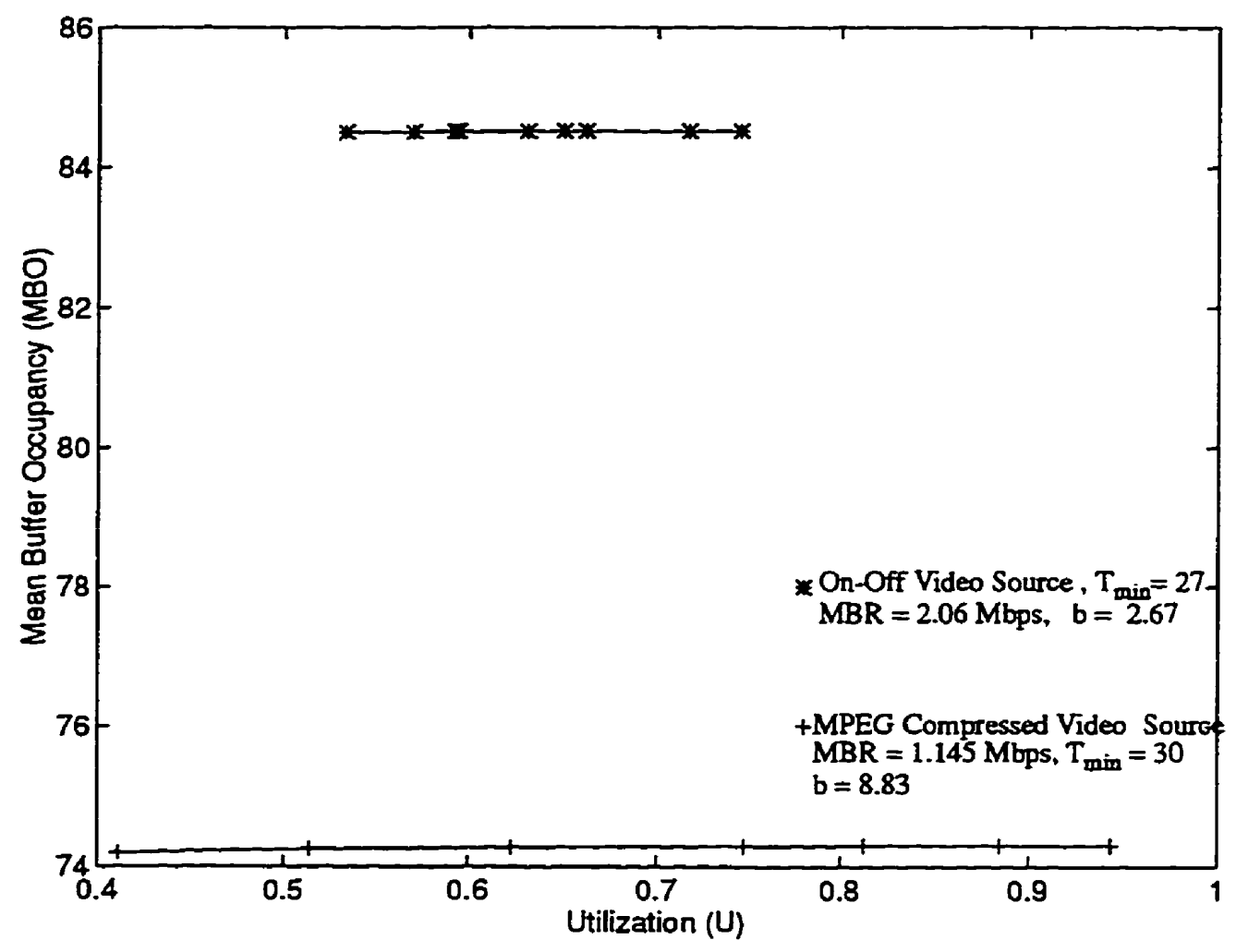

Figure 5-22: Mean Buffer Occupancy versus Utilization for VBR MPEG Compressed Video Source. CFR $=4.94 \%$, MTD $=33 \mathrm{msec}$. 


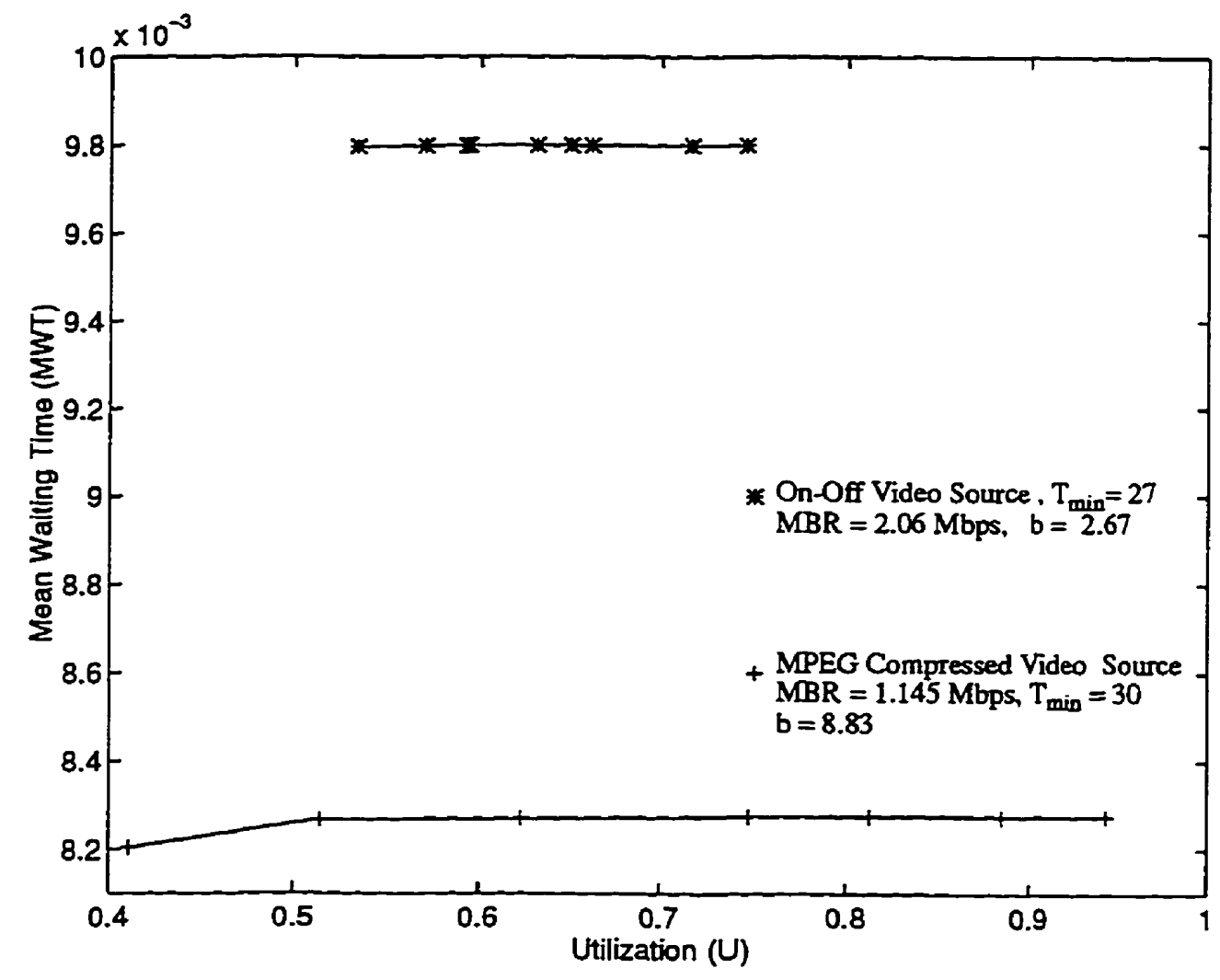

Figure 5-23: Mean Waiting Time versus Utilization for VBR MPEG Compressed Video Source. CFR $=4.94 \%$, MTD $=33 \mathrm{msec}$ 


\section{Chapter 6}

\section{Conclusions}

\subsection{Conclusions}

The scope of this thesis was to describe and implement a simplified simulation strategy for a BIWN in order to determine the steady state network performance of a BIWN as seen by a given user terminal. This work covers three interesting areas: broadband indoor wireless local area networks, development of a simulation scheme and modeling of multimedia traffic. The studied system is characterized by channel impairments, traffic heterogeneity and diverse users' quality of service (QOS) requirements. We focussed on variable bit rate video traffic. We investigated such a system by considering the radio channel behaviour, traffic characteristics and QOS and incorporating them into a simulation model. For this purpose, we considered the radio channel as an ON/OFF link, characterized by the average channel failure rate (CFR).

In Chapter 3, we showed that the indoor channel behaviour can be approximated by a fade/non-fade (ON/OFF) model with exponentially distributed ON and OFF states. Later, in the same chapter we proposed a similar ON/OFF channel model which considered the effects of fading, shadowing and interference separately. The model was based on the fact 
that the distribution of the ON and OFF state intervals of the ON/OFF model is a function of the effectiveness of the techniques used to combat fading, shadowing and interference.

We have assumed that the communicating remotes generate highly bursty, sensitive to delay traffic. To simulate such traffic, we used:

a) ON/OFF Markov mini-sources model.

b) Variable Bit Rate Compressed Video Data based on models exhibiting self-similar characteristics, both described in Chapter 4. Two independent sets of VBR Video data, with a wide range of burstiness characteristics were generated using the above two models, in order to represent the video traffic in a BIWN system.

The simulation results presented in Chapter 5 clearly prove that the steady state system performance is dependent upon i) the nature of the video traffic, ii) the minimum guaranteed bandwidth $T_{\min }$, iii) the channel failure rate (CFR) and iv) the MTD for the given user.

Using the data obtained from the ON/OFF Markov mini-sources model (described in Chapter 4 and 5) to represent the overall cell traffic in the system under consideration, the effect of variation of $\mathrm{T}_{\min }$ assigned to a given user was evaluated by simulations. The system exhibited poorer steady state performance with decreasing values of $T_{\min }$. Higher values of $\mathrm{T}_{\min }$ resulted in low CLP, MBO and MWT values for a given value of the offered load. However, due to the high time sensitivity of the source the increase in the MBO and the MWT is minimal (leaky bucket principle). The increase in the MBO and the MWT is more prominent with a higher MTD (lower time sensitivity).

Simulations were carried out for two different values of MTD, keeping other parameters fixed. It was observed that a source with a higher MTD requires a lower $T_{\min }$ than a source 
with a lower MTD to give a required steady state performance. Further, as the MTD of a given video source is increased (time sensitivity lowered), a given change in the $T_{\text {min }}$ assigned to the terminal has a lower impact on the steady state performance of the terminal. In other words, assignment of a proper $T_{\min }$ becomes extremely important for highly time sensitive sources. For such sources, even a slightly lower $T_{\min }$ than required, can result in a very high cell loss probability. In case of less time sensitive sources, assignment of a low $\mathrm{T}_{\min }$ results in a higher MBO and MWT as compared to the more time sensitive sources.

Further, with the same video sources, simulations were carried out to study the effect of variation of MTD for the given user traffic, keeping all other simulation parameters fixed. The CLP was found to decrease with an increase in the MTD, giving improved performance as expected due to lower time sensitivity of the video cells. MBO and MWT were found to increase with an increase in the MTD.

Another set of simulations was carried out using VBR Compressed video data exhibiting self-similar characteristics. The effect of variation of the MTD was considered again. Similar results as in the previous case were obtained except that the difference between the steady state values of the latter was observed to be much greater than that of the former. This phenomenon can be attributed to a considerably higher burstiness in the given user's video traffic in case of the latter. Later, the effect of variation of the average CFR on the performance curves was studied by altering the channel model parameters in the simulation model. As expected, CLP, MBO and the MWT were found to increase with an increase in the average CFR. We have carried out simulations for three different sets of values assigned to the transition probabilities of the channel model. However, simulations can be run by plugging in a range of values of the transition probabilities into the channel model to study the effect of shadowing, fading and interference in greater details. 
The impact of change in the network capacity on the steady state performance was also evaluated by simulations. It was interesting to note how the variation in the network capacity affected the performance curves. CLP, MBO and MWT varied differently with utilization in each of the cases. Steady state performance was obtained at different values of utilization for the three cases. The performance curves obtained can be utilized to determine the number of users with known traffic characteristics that can be supported by a given network and vice-versa.

We finally compared the steady state performance exhibited by two video sources with varying traffic characteristics. One of the sources had a significantly higher level of burstiness but a lower MBR than the other. The former exhibited a much higher CLP for a given value of utilization than the latter despite the fact that the former was assigned a higher $T_{\min }$ than the latter. This highlights the crucial role of burstiness of a traffic source in influencing the steady state performance of a bursty user.

From the previously stated results we realize that communicating remotes consisting of bursty and sensitive to delay sources present a poor steady state performance under conditions of improper bandwidth allocation. Further, the obtained results also help us in determining the upper bound on utilization which guarantees users' QOS requirements. We conclude that proper allocation of $\mathrm{T}_{\min }$ or the minimum guaranteed bandwidth must be made to the remotes depending on the parameters declared by those terminals to the controller viz. burstiness, mean bit rate (MBR), MTD etc.

Summing up, our simplified simulation strategy for a BIWN, using which we determined the steady state network performance as seen by a given terminal, offers the following advantages:

a) It is much simpler than simulating the traffic flow in the entire system. 
b) It gives a good approximation of a full system simulation.

c) It determines the performance of a given type of traffic in a heterogeneous network.

We carried out a simulation study for video traffic and compared the performance curves by varying $T_{\min }, M T D, C F R$, the network capacity and the burstiness of the given video source. The same approach can be applied to obtain results for voice and data. The developed simulation model can be conveniently used to determine the maximum number of users that can be supported by a BIWN system, satisfying the QOS requirements, and vice-versa. The model helps in ariving at a realistic scenario of the overall system based on the system performance seen by the given user terminal. This didn't require detailed simulation of any subsystem, however details can be added later on.

In order to obtain the required performance curves, the simulation must be run to have at least 100 errors at the lowest probability of predicted enrors. In other words 10000000 cells must be transmitted by the video source in the simulation to be able to get a CLP of $10^{-5}$. The simulations were conducted with a limited number of video data files (representing the video sources used in the simulations). Hence there could be statistical anomalies that are not representative of other video sources but specific to the ones used.

\subsection{Future Research}

The objective of this work was to develop a simplified simulation approach to evaluate the performance of an indoor wireless network supporting high speed bursty traffic. We used simplified models for the radio channel and the input bursty traffic. Further research must be carried out to characterize the ON and OFF periods accurately using experimental results. Factors such as imperfect transmission of control messages, interaction between downlink and uplink traffic and implementation limitations must be considered. The details of fade combatting techniques must also be considered in future research. 
We carried out an investigation for a single cell scenario, considering the radio link characteristics, the effect of CCI, traffic heterogeneity and QOS parameters. This work can be extended to consider the effects of handoff. This would give a more comprehensive and a thorough view of the system as seen by a given user. A greater emphasis must be laid on generating multimedia cell traffic over a large period of time and a wide range of magnitudes using an accurately designed model. One approach to achieve this task would be to determine the average traffic generated by a VBR Video terminal based on a collection of independent samples of a large number of diverse video sources. A filter must be accurately designed using the long term autocorrelation statistics of the average video traffic. This filter when fed with uncorrelated data samples of a certain mean and variance and which follow a given distribution (say Gaussian distribution) shall generate VBR Video data. 


\section{References}

[1] Saleh A.A.M. and L.J. Cimini, Jr., "Indoor Radio Communications Using TimeDivision Multiple Access With Cyclical Slow Frequency Hopping and Coding," ICC ‘88, Vol.2, pp. 774-780, June 1988.

[2] J.B. Andersen, Theodore S. Rappaport, and Susumu Yoshida, "Propagation Measurements and Models for Wireless Communications Channels," IEEE Communications Magazine, pp. 42 - 49, Jan.1995

[3] M.R. Finley Jr., A. Karakura and R. Nbogni, "Survey of Intelligent Building Concepts," IEEE Communications Magazine, pp. 18-23, Apr. 1991.

[4] Kaveh Pahlavan, Thomas H. Probert, and Mitchell E. Chase, "Trends in Local Wireless Networks," IEEE Communications Magazine, pp. 88-95, March 1995.

[5] F.R. Gfeller and U. Bapst, "Wireless in-house data communication via diffuse infrared radiation," Proc.IEEE, vol.67, Nov.1979, pp.1474-1486.

[6] P. Ferert, "Application of spread spectrum radio to wireless terminal communications," Proc. IEEE NTC '80, Houston, TX, 1980, pp. 244-248.

[7] K. Pahlavan, "Wireless office information networks," IEEE Communications Magazine, vol.23, no.6, June 1985, pp. 19-27.

[8] C.S. Yen and R.D. Crawford, "The use of directed beams in wireless computer commuications," Proc. IEEE GLOBECOM '85, Dec.1985,pp.1181-1184.

[9] Y. Nakata et al., "In-house wireless communication systems using infrared radiations," Proc. Int. Conf. Comp. Commun., Sydney, Australia, 1984, pp. 333-338.

[10] M.J. Marcus, "Regulatory policy considerations for radio local area networks," IEEE Communications Magazine, vol.25, no.7, July 1987, pp.95-99.

[11] T. Freeburg, "A new technology for high speed wireless local area networks," Proc. IEEE Workshop on Wireless LANs, Worcester, MA, May 1991, pp. 127-139. 
[12] T.A. Freeburg, "Enabling technologies for Wireless In-building Network Communications-Four technical challenges, Four solutions," IEEE Communications Magazine, pp.58, April 1991.

[13] Jay E. Padgett, Christoph G. Günther, and Takeshi Hattori, "Overview of Wireless Personal Communications," IEEE Communications Magazine, pp. 28-41, Jan 1995.

[14] D.J. Goodman, "Trends in cellular cordless communications," IEEE Communications Magazine, pp.31, June 1991.

[15] J.E. Mitzlaff, "Radio propagation and anti-multipath techniques in the WIN environment", IEEE Network Magazine, p.21, Nov. 1991

[16] R.M. Rodriguez-Dagnino \& A. Leon-Garcia, "Modelling VBR video sequences containing scene transitions: A case study", Tech. Report, University of Toronto, Dept of Electrical Engineering, May 1992.

[17] V.R.M. Thyagarajan, "A simulation traffic study of indoor wireless communications in the (20-60) GHz band", Thesis for Master of Engineering, Dept. of Systems and Computer Engineering, Carleton University, 1993.

[18] R. Prasad \& L. Vandendorpe, "An overview of millimeter wave indoor wireless communication systems," p. 885, ICUPC, Ottawa, Oct. 1993.

[19] R.Prasad \& W. Bredero, "Comparison of protocols in macro, micro and pico cellular environment for millimetric wavelength," COST231 contribution, Barcelona, Jan.1993.

[20] R.Sonnemans, "ATM in an indoor radio network: A feasibility study," graduation report, Eindhoven University of Technology, Faculty of Electrical Engineering, Aug.1990.

[21] R.B. Maessen, "Use of ATM in an in-house radio network," graduation report, Eindhoven university of technology, Faculty of Electrical Engineering, June 1992.

[22] D.M. Pozar, Microwave Engineering, Addison Wesiey series in Electrical and Computer Engineering, Massachusets, 1990.

[23] Cox D.C., "Wireless Network Access for Personal Communications," IEEE Communications Magazine, pp. 96-106, Dec. 1992.

[24] Falconer D.D., F. Adachi, B. Gudmundson, "Time Division Multiple Access Methods for Wireless Personal Communications," IEEE Communications Magazine, pp. 50-57, January 1995. 
[25] Zhang C.G., D.D. Falconer and H.M. Hafez, "Traffic Characteristics of an Indoor Wireless Broadband Network Using CDMA Multiple Access," Wireless '93 Conference Record, Wireless '93, Calgary, July 1993.

[26] Falconer D.D., H.M. Hafez, and S.A. Mahmoud, "Microcellular Architecture," Carleton University Internal Report, 1994.

[27] Movahhedinia N., G. Stamatelos, and H.M. Hafez, "Bandwidth Management in Indoor Broadband Wireless Networks (IBWN)", Ph.D Thesis Report, Carleton University, 1996.

[28] Ferrari D., "Client Requirements for Real-Time Communication Services," IEEE Communications Magazine, pp. 65-72, Nov. 1990.

[29] Gallassi G., G. Rigolio, and L. Verri, "Resource Management and Dimensioning in ATM networks," IEEE Network Magazine, pp. 8-17, May 1990.

[30] IEEE T1P1 - Personal Communications, "IEEE 802.11 Wireless Local Area Network Requirements," Committee T1 Contribution, T1P1 2/92-059, April 1992.

[31] Kessler G. C., ISDN Second Edition, McGraw-Hill Inc. 1993.

[32] Heinrich Armbruster, "The Flexibility of ATM: Supporting Future Multimedia and Mobile Communications", IEEE Communications Magazine, p.82, Feb 1995.

[33] D.Anderson \& J.Molyneaux, "Three technical hurdles on the road to a wireless office:Integration,Integration,Integration",Proc.IEEE Int. Conf. on Selected Topics in Wireless Communications, Vancouver, June 1992.

[34] Saleh A. A. M. and L.J. Cimini, Jr., "Indoor Radio Communications Using TimeDivision Multiple Access With Cyclical Slow Frequency Hopping and Coding," IEEE JSAC, Vol.7, Iss.1, pp.59-70, Jan. 1989.

[35] W.C. Jakes, Microwave Mobile Communications, Wiley Interscience Publications, New York, 1974.

[36] Linnartz J.P., Narrowband Land-Mobile Radio Networks, Artech House, 1993.

[37] A.S. Mahmoud, David D. Falconer and Samy A. Mahmoud, “ A Multiple Access Scheme for Wireless Access to a Broadband ATM LAN Based on Polling and Sectored Antennas," submitted to IEEE JSAC, 1995.

[38] Daigle, and Langford, "Queueing Analysis of a packet voice communication system," IEEE JSAC, vol. SAC-4, No.6, Sept.1986. 
[39] Maglaris B., K. Anastassiou, P. Sen, G. Karlsson, and J.D. Robbins, "Performance Models of Statistical Multiplexing in Packet Video Communications," IEEE Trans. on Comm., VOL.36, NO.7, pp.834-843, July 1988.

[40] Nomura M., T. Fujii, and N. Ohta, "Basic Characteristics of Variable Rate Video Coding in ATM Environment," IEEE JSAC, VOL.7, NO.5, pp. 752-760, June 1989.

[41] Melamed B., "TES: A Class of Methods for Generating Autocorrelated Uniform Variates," ORSA J. Computing, 3,4, pp. 317-329, 1991.

[42] Jagerman K.L., and B. Melamed, "The Transition and Autocorrelation Structure of TES Processes Part I: General Theory," Stochastic Models, 8, 2, pp. 193-219, 1980.

[43] Marafih N.M., Y. Zhang, R.L. Pickholtz, "Modeling and Queueing Analysis of Variable-Bit-Rate Coded Video Sources in ATM Networks," IEEE ICC ' 92.

[44] Sen P., B. Maglaris, N. Rikli, and K. Anastassiou, "Models for Packet Switching of Variable-Bit-Rate Video Sources," IEEE JSAC, VOL.7, NO.5, pp. 865-869, June 1989.

[45] Sohraby K., "On the Theory of General ON-OFF Sources With Applications in High-Speed Networks," INFOCOM '93, pp. 401-409.

[46] Yasuda Y., H. Yasuda, N. Ohta, and F. Kishino, "Packet Video Transmission Through ATM Networks," IEEE GLOBECOM '89, Vol.2, pp. 25.1.1-25.1.5, Nov.1989.

[47] Heyman D., A. Tabatabai, and T.V. Lakshman, "Statistical Analysis and Simulation Study of Video Teleconference Traffic in ATM Networks," IEEE Trans. on Circuits and Systems for Video Tech., VOL.2, NO.1, pp. 49-59, Mar. 1992.

[48] Li S.Q. and H.D. Sheng, "Discrete Queueing Analysis of Multimedia Traffic with Diversity of Correlation and Burstiness Properties," IEEE Transactions on Communications, Vol.42, No. 2/3/4, pp. 1339-1353, April 1994.

[49] Bae J.J., and T. Suda, "Survey of Traffic Control Schemes and Protocols in ATM Networks," IEEE Proceedings, VOL.79, NO.2, pp. 170-189, Feb.1991.

[50] Jou Y. F., A.A. Nilsson, and F. Lai, "The Upper bounds for Performance Measures of a Finite Capacity Polling System Under Bursty Arrivals," Queueing Networks With Finite Capacity, Elsevier Science Publishers, pp. 83-96, 1993.

[51] Kowtha S., D.R. Vaman, " A Generalized ATM Traffic Model and its Application in Bandwidth Allocation," ICC ‘92, Vol.2, pp. 1009-1013, June 1992. 
[52] Verbiest W., and L. Pinnoo, "A Variable Bit Rate Video Codec for Asynchronous Transfer Mode Networks," IEEE JSAC, VOL.7, NO.5, pp.761-770, June 1989.

[53] J. Beran, R. Sherman, M.S. Taqqu, and W. Willinger, "Long-Range Dependence in Variable-Bit-Rate Video Traffic," To appear on IEEE Transactions on Communications, 1994.

[54] B.B. Mandelbrot, The Fractal Geometry of Nature. Freeman, 1983.

[55] W.E. Leland, M.S. Taqqu, W. Willinger, and D.V. Wilson, "On the Self-Similar Nature of Ethemet Traffic (Extended Version)," ACM/IEEE Transactions on Networking, 2(1):1-15, Feb.1994.

[56] J.R.M. Hosking. Modeling Persistence in Hydrological Time Series Using Fractional Differencing. Water Resources Research, 20(12):1898-1908, 1984.

[57] C. Huang, M. Devetsikiotis, I. Lambadaris, A.R. Kaye. Modeling and Simulation of Self-Similar Variable Bit Rate Compressed Video: A Unified Approach. In Proc. ACM SIGCOMM '95, pages 114-125, Cambridge, Massachusetts, August 1995.

[58] D. Legall, "MPEG: A Video Compression Standard for Multimedia Applications," Communications of the ACM, 34(4), Apr.1991.

[59] ISO. MPEG-1 Specification. CD11172.

[60] Sun Microsystems Computer Corporation. Sun Video 1.0 User's Guide, Oct. 1993.

[61] Portable Video Research Group, Stanford University. PVRG-MPEG Codec 1.1, June 1993. 